\title{
On Smooth Solutions to the Thermostated Boltzmann Equation with Deformation
}

\author{
Renjun Duan ${ }^{1}$ and Shuangqian $\mathrm{Liu}^{2, *}$ \\ ${ }^{1}$ Department of Mathematics, The Chinese University of Hong Kong, Shatin, \\ Hong Kong, SAR, P.R. China. \\ 2 School of Mathematics and Statistics, Central China Normal University, \\ Wuhan 430079, P.R. China.
}

Received 20 November 2021; Accepted 1 December 2021

\begin{abstract}
This paper concerns a kinetic model of the thermostated Boltzmann equation with a linear deformation force described by a constant matrix. The collision kernel under consideration includes both the Maxwell molecule and general hard potentials with angular cutoff. We construct the smooth steady solutions via a perturbation approach when the deformation strength is sufficiently small. The steady solution is a spatially homogeneous non Maxwellian state and may have the polynomial tail at large velocities. Moreover, we also establish the long time asymptotics toward steady states for the Cauchy problem on the corresponding spatially inhomogeneous equation in torus, which in turn gives the non-negativity of steady solutions.
\end{abstract}

AMS subject classifications: 35Q20, 35B40

Key words: Boltzmann equation, deformation force, thermostated force, non-equilibrium steady state, asymptotic stability.

\section{Introduction}

The homoenergetic solutions to the Boltzmann equation were first introduced by Galkin [19] and Truesdell [28] independently at almost the same time. These

*Corresponding author. Email addresses: rjduan@math. cuhk. edu.hk (R.-J. Duan), tsqliu@jnu. edu.cn (S.-Q. Liu) 
prototypical solutions not only indicate the existence of invariant manifolds of molecular dynamics but also give a new insight into the relation between atomic forces and nonequilibrium behavior of the gas. Recently, James et al. [25-27] and Bobylev et al. [10] provided the systematic mathematical study of the subject. Motivated by those works, the authors of this paper also considered the smoothness and asymptotic stability of self-similar solutions to the Boltzmann equation for the uniform shear flow in case of the Maxwell molecule [14]. In the non Maxwell molecule case, for instance, for the hard potentials, the problem is more subtle to treat and still remains largely open, because the temperature of system increases only in a polynomial rate depending on the collision kernel and the shear rate in the rescaled equation is no longer a constant but a time-dependent function, see the conjecture in [25] for details.

On the other hand, instead of studying the uniform shear flow as a timedependent state due to the viscous heating, it is also usual to introduce nonconservative external forces to compensate exactly for the viscous increase of temperature and achieve a steady state. This kind of force is referred to as thermostats and a typical choice of the thermostat force is the friction $-\beta v$ with a constant $\beta \in \mathbb{R}$, see [20, Chapter 3.4]. Inspired by this, we are concerned in this paper with the spatially homogeneous steady problem on the thermostated Boltzmann equation with a deformation force

$$
-\beta \nabla_{v} \cdot\left(v G_{s t}\right)-\alpha \nabla_{v} \cdot\left(A v G_{s t}\right)=Q\left(G_{s t}, G_{s t}\right) .
$$

Here, the unknown $G_{s t}=G_{s t}(v)$ denotes the non-negative velocity distribution function of particles with velocity $v \in \mathbb{R}^{3}$. The matrix $A=\left(a_{i j}\right) \in M_{3 \times 3}(\mathbb{R})$ induces a deformation force $-\alpha A v$ with the strength given by the parameter $\alpha>0$ and the constant $\beta \in \mathbb{R}$ is a parameter standing for the strength of the thermostated force. The nonlinear term $Q(\cdot, \cdot)$ is the collision operator defined as

$$
Q\left(F_{1}, F_{2}\right):=\int_{\mathbb{R}^{3}} \int_{\mathbb{S}^{2}} B\left(\omega, v-v_{*}\right)\left[F_{1}\left(v_{*}^{\prime}\right) F_{2}\left(v^{\prime}\right)-F_{1}\left(v_{*}\right) F_{2}(v)\right] d \omega d v_{*},
$$

where we have denoted $v^{\prime}=v+\left[\left(v_{*}-v\right) \cdot \omega\right] \omega$ and $v_{*}^{\prime}=v_{*}-\left[\left(v_{*}-v\right) \cdot \omega\right] \omega$ with $\omega \in \mathbb{S}^{2}$ in terms of the conservation laws $v_{*}+v=v_{*}^{\prime}+v^{\prime}$ and $\left|v_{*}\right|^{2}+|v|^{2}=\left|v_{*}^{\prime}\right|^{2}+\left|v^{\prime}\right|^{2}$. Throughout this paper, we let

$$
\begin{aligned}
& B\left(\omega, v-v_{*}\right)=\left|v-v_{*}\right|^{\gamma} B_{0}(\cos \theta), \quad \cos \theta=\omega \cdot \frac{v-v_{*}}{\left|v-v_{*}\right|}, \quad \omega \in \mathbb{S}^{2}, \\
& 0 \leq \gamma \leq 1, \quad 0 \leq B_{0}(\theta) \leq C|\cos \theta| .
\end{aligned}
$$

This includes the cases of the Maxwell molecule $\gamma=0$ and general hard potentials $0<\gamma \leq 1$ under the Grad's angular cutoff assumption. 
To consider (1.1), we supplement it with the restriction condition

$$
\int_{\mathbb{R}^{3}}\left[1, v,|v|^{2}\right] G_{s t}(v) d v=[1,0,3] .
$$

The steady problem (1.1) is solvable only if its left hand term is microscopic, namely,

$$
\int_{\mathbb{R}^{3}}\left[1, v,|v|^{2}\right]\left\{-\beta \nabla_{v} \cdot\left(v G_{s t}\right)-\alpha \nabla_{v} \cdot\left(A v G_{s t}\right)\right\} d v=0 .
$$

This together with (1.5) implies that

$$
\beta=-\alpha \frac{\int_{\mathbb{R}^{3}} v \cdot(A v) G_{s t} d v}{\int_{\mathbb{R}^{3}}|v|^{2} G_{s t} d v}=-\frac{\alpha}{3} \int_{\mathbb{R}^{3}} v \cdot(A v) G_{s t} d v .
$$

Plugging this back to (1.1) gives

$$
\frac{1}{3} \int_{\mathbb{R}^{3}} v \cdot(A v) G_{s t} d v \nabla_{v} \cdot\left(v G_{s t}\right)-\nabla_{v} \cdot\left(A v G_{s t}\right)=\frac{1}{\alpha} Q\left(G_{s t}, G_{s t}\right) .
$$

From (1.7), the deformation strength $\alpha>0$ plays the same role as the Knudsen number, and we then expect to adopt the perturbation approach as in [14] to construct smooth solutions for any small $\alpha>0$.

To present the main results of this paper, we first introduce some notations. To this end, associated with the condition (1.5), we define the reference global Maxwellian $\mu$ by

$$
\mu=(2 \pi)^{-\frac{3}{2}} e^{-\frac{|v|^{2}}{2}},
$$

and use the velocity weight function

$$
w_{l}=w_{l}(v):=\left(1+|v|^{2}\right)^{l}
$$

with an integer $l>0$. Let $\zeta=\left(\zeta_{1}, \zeta_{2}, \zeta_{3}\right)$ and $\vartheta=\left(\vartheta_{1}, \vartheta_{2}, \vartheta_{3}\right)$ be multi-indices with length $|\zeta|$ and $|\vartheta|$, respectively, and write

$$
\partial_{\zeta}=\partial_{v}^{\zeta}=\partial_{v_{1}}^{\zeta_{1}} \partial_{v_{2}}^{\zeta_{2}} \partial_{v_{3}}^{\zeta_{3}}, \quad \partial^{\vartheta}=\partial_{x}^{\vartheta}=\partial_{x_{1}}^{\vartheta_{1}} \partial_{x_{2}}^{\vartheta_{2}} \partial_{x_{3}}^{\vartheta_{3}}, \quad \partial_{\zeta}^{\vartheta}=\partial_{v}^{\zeta} \partial_{x}^{\vartheta},
$$

for simplicity. We define $\zeta^{\prime} \leq \zeta$ if each component of $\zeta^{\prime}$ is not greater than the one of $\zeta$, and write $\zeta^{\prime}<\zeta$ in case of $\zeta^{\prime} \leq \zeta$ and $\left|\zeta^{\prime}\right|<|\zeta|$. We also let $C_{\vartheta}^{\vartheta^{\prime}}$ be the usual binomial coefficient for two multi-indices $\vartheta$ and $\vartheta^{\prime}$ with $\vartheta^{\prime} \leq \vartheta$.

The first result in the paper is to establish the existence of smooth solutions to the steady problem (1.1) and (1.5) for the steady state $G_{s t}$ with $\beta$ given by (1.6) . 
Theorem 1.1. Assume (1.3) and (1.4) for the collision kernel. Let $A=\left(a_{i j}\right) \in M_{3 \times 3}(\mathbb{R})$ be a non scalar matrix and

$$
G_{1}=-\sum_{i, j=1}^{3} a_{i j} L^{-1}\left\{\left(v_{i} v_{j}-\frac{1}{3} \delta_{i j}|v|^{2}\right) \mu^{\frac{1}{2}}\right\},
$$

where $L$ is the linearized collision operator as in (2.1). Then, there is an integer $l_{0}>0$ such that for any integer $l \geq l_{0}$, there is $\alpha_{0}=\alpha_{0}(l)>0$ depending on $l$ such that for any $\alpha \in\left(0, \alpha_{0}\right)$, the steady problem (1.1) and (1.6) under the condition (1.5) admits a unique non-negative smooth solution $G_{s t}=G_{s t}(v) \in C^{\infty}\left(\mathbb{R}^{3}\right)$ of the form

$$
G_{s t}=\mu+\alpha \mu^{\frac{1}{2}} G_{1}+\alpha^{2} \widetilde{G}_{R},
$$

such that

$$
\int_{\mathbb{R}^{3}}\left[1, v,|v|^{2}\right] \widetilde{G}_{R} d v=0
$$

and for any integer $m \geq 0$,

$$
\sum_{|\zeta| \leq m}\left\|w_{l} \partial_{\zeta} \widetilde{G}_{R}\right\|_{L^{\infty}} \leq \tilde{C}_{m, l}
$$

where $\tilde{C}_{m, l}>0$ is a constant depending only on $m$ and $l$ but not on $\alpha$ and $w_{l}$ is given in (1.9).

Similar to [14], we point out that the obtained steady solution is a non Maxwellian state and may have the polynomial tail at large velocities, which is the main feature of the problem. In order to justify the non-negativity of the steady solution $G_{s t}$ constructed in Theorem 1.1, we introduce a spatially inhomogeneous model in torus $\mathbb{T}^{3}=[0,2 \pi]^{3}$

$$
\begin{gathered}
\partial_{t} G+v \cdot \nabla_{x} G-\beta \nabla_{v} \cdot(v G)-\alpha \nabla_{v} \cdot(A v G) \\
=Q(G, G), \quad t>0, \quad x \in \mathbb{T}^{3}, \quad v \in \mathbb{R}^{3}, \\
G(0, x, v)=G_{0}(x, v), \quad x \in \mathbb{T}^{3}, \quad v \in \mathbb{R}^{3} .
\end{gathered}
$$

It turns out that the steady solution $G_{s t}$ can be used to describe the large time asymptotics of the unsteady problem (1.13) and (1.14). We state this result as follows.

Theorem 1.2. Let $G_{s t}(v)$ be the steady profile obtained in Theorem 1.1 and the constant $\beta$ be defined in (1.6). Assume further that $\beta I+\alpha A$ is invertible. Then, there are constants $\lambda>0$ and $C>0$ independent of $\alpha$ such that if it holds that $G_{0}(x, v) \geq 0$,

$$
\int_{\mathbb{T}^{3}} \int_{\mathbb{R}^{3}}\left[G_{0}(x, v)-G_{s t}\right] d v d x=0, \quad \int_{\mathbb{T}^{3}} \int_{\mathbb{R}^{3}} v G_{0}(x, v) d v d x=0,
$$


and

$$
\sum_{|\zeta|+|\vartheta| \leq m}\left\|w_{l} \partial_{\zeta}^{\vartheta}\left[G_{0}(x, v)-G_{s t}(v)\right]\right\|_{L^{\infty}} \leq \alpha^{2},
$$

for an integer $m \geq 1$, then the Cauchy problem (1.13)-(1.14) admits a unique global solution $G(t, x, v) \geq 0$ such that

$$
\sum_{|\zeta|+|\vartheta| \leq m}\left\|w_{l} \partial_{\zeta}^{\vartheta}\left[G(t, x, v)-G_{s t}(v)\right]\right\|_{L^{\infty}} \leq C \alpha e^{-\lambda \beta_{1} \alpha^{2} t}
$$

for any $t \geq 0$, where $\beta_{1}>0$ is a positive constant given by

$$
\beta_{1}=-\frac{1}{3} \int_{\mathbb{R}^{3}} v \cdot(A v)\left\{\mu^{\frac{1}{2}} G_{1}+\alpha \widetilde{G}_{R}\right\} d v .
$$

In what follows we mention some existing works that are most related to the background and motivations of the current topic; readers may refer to [14] for a more detailed review. Based on the Fourier transform method in $[4,5]$, Bobylev and Cercignani [7-9] discussed the self-similar asymptotics for the spatially homogeneous Boltzmann equation. As in the original work by Galkin [19] and Truesdell [28], by solving the ODE system consisting of velocity moments, particularly the second order moments, Cercignani [13] investigated the shear flow problem on a granular flow between parallel plates which is modeled by the Boltzmann equation, and Bobylev and Cercignani [6] later obtained the well-posedness and large time behavior of the granular system described by Boltzmann-like equations. We also mention that Cercignani [12] proved the global existence of homoenergetic affine flows for the Boltzmann equation in the case of simple shear for a large class of interaction potentials which include hard potentials, and these solutions in general may not be self-similar. It seems that [12] is the first mathematical result on the homoenergetic solution of the Boltzmann equation for the non Maxwell molecule.

Recently, in a significant progress by James et al. [25], the existence of homoenergetic mild solutions as non-negative Radon measures was studied in a systematic way for a large class of initial data, and the problem on the asymptotics of homoenergetic solutions in the case of non Maxwell molecules was also proposed. In the meantime, it is discussed in $[26,27]$ that there is a balance between the hyperbolic term and collision term for the Boltzmann equation describing homogenergetic flow and the corresponding long time asymptotic behavior depends on which term is dominated in large time. By combining the Fourier transform method and moments argument, a more recent progress has been achieved by Bobylev et al. [10], where the authors proved the self-similar asymptotics of solutions in large time for the Boltzmann equation with a general deformation force 
under a smallness condition on the matrix $A$, and they also showed that the selfsimilar profile can have the finite polynomial moments of higher order as long as the norm of $A$ is getting smaller. To the best of our knowledge, [10] seems the only known result on the large time asymptotics to the self-similar profile in weak topology, see also [3] for a further study to provide explicit estimates of the smallness of the matrix $A$. Following $[10,25]$, in the case of Maxwell molecule, the authors of this paper [14] constructed smooth self-similar profiles for the shear flow problem on the Boltzmann equation and proved the dynamical stability of the stationary solution via a perturbation approach.

As mentioned at the beginning, different from the uniform shear flow where the temperature increases in time and the self-similar asymptotic has to be involved, we expect the extra thermostated term to compensate the viscous heating energy and drive the system to converge to the steady state. We remark that a similar situation may occur to the bounded domain case with diffuse boundaries that also can absorb the shearing energy such that the system tends asymptotically to the steady motion instead of the self-similar solution. In particular, a boundary value problem on the Boltzmann equation for the plane Couette flow was studied in [15], where they established the existence of spatially inhomogeneous non-equilibrium stationary solutions to the steady problem for small shear rate and proved dynamical stability of the stationary solution.

Compared to our previous work [14] about the self-similar steady problem in case of the simple shear force and Maxwell molecules, we treat in this paper the more general deformation force described by the matrix $A$ and also include the case of hard potentials $0<\gamma \leq 1$ for the molecular interaction. In what follows we outline the key strategies in the proof of main results and point out the main differences with [14]. First of all, for the steady problem (1.1) or (1.7), we look for solutions by setting the perturbation $G_{s t}=\mu+\alpha \sqrt{\mu} G_{1}+\alpha^{2} \widetilde{G}_{R}$ with $\widetilde{G}_{R}=\sqrt{\mu} G_{R}$ as in (1.11). Here, $G_{1}$ as in (1.10) is introduced to remove the zero-order inhomogeneous term in terms of (2.13) and $G_{R}$ is the remainder satisfying (2.14). Note that $G_{1}$ involves the general deformation matrix $A$ and it is non-zero for any non scalar matrix $A$. The usual energy approach fails to be used to treat (2.14) due to the second order velocity growth of the term $\frac{\alpha}{2} v \cdot(A v) G_{R}$ since the linearized collision operator only provides the dissipation term $\int v(v)\left|G_{R}\right|^{2} d v$ with $v(v) \sim|v|^{\gamma}$ $(0 \leq \gamma \leq 1)$ for large velocities. As in [14], we employ the Caflisch's decomposition (cf. [11])

$$
\widetilde{G}_{R}=\sqrt{\mu} G_{R}=G_{R, 1}+\sqrt{\mu} G_{R, 2}
$$

where $G_{R, 1}$ and $G_{R, 2}$ satisfy the coupled system

$$
-\alpha^{2} \beta_{1} \nabla_{v} \cdot\left(v G_{R, 1}\right)-\alpha \nabla_{v} \cdot\left(A v G_{R, 1}\right)+v G_{R, 1}
$$




$$
=\chi_{M} \mathcal{K} G_{R, 1}-\frac{1}{2} \alpha^{2} \beta_{1}|v|^{2} \sqrt{\mu} G_{R, 2}-\frac{\alpha}{2} v \cdot(A v) \sqrt{\mu} G_{R, 2}+\cdots
$$

and

$$
-\alpha^{2} \beta_{1} \nabla_{v} \cdot\left(v G_{R, 2}\right)-\alpha \nabla_{v} \cdot\left(A v G_{R, 2}\right)+L G_{R, 2}=\mu^{-\frac{1}{2}}\left(1-\chi_{M}\right) \mathcal{K} G_{R, 1},
$$

respectively. The benefit of this splitting is that the term $\frac{\alpha}{2} v \cdot(A v) \sqrt{\mu} G_{R, 2}$ is no longer a trouble since it contains $\sqrt{\mu}$ which can absorb any order polynomial velocity growth. The price to pay is that one cannot make a direct energy estimate on $G_{R, 1}$ because $\chi_{M} \mathcal{K} G_{R, 1}$ may not be small in the $L^{2}$ setting. However, this can be resolved in terms of the $L^{2}-L^{\infty}$ interplay since the smallness for $\chi_{M} \mathcal{K} G_{R, 1}$ can be recovered via the velocity weighted $L^{\infty}$ norm. Indeed, in the case of Maxwell molecule, the following decay mechanism of $\mathcal{K}$ has been found in [14]:

$$
\sup _{|v| \geq M(l)} w_{l}\left|\partial_{\zeta}(\mathcal{K} f)\right| \leq \frac{C}{l} \sum_{0 \leq \zeta^{\prime} \leq \zeta}\left\|w_{l} \partial_{\zeta^{\prime}} f\right\|_{L^{\infty}}
$$

where $C$ is independent of $l$ and $M(l) \rightarrow \infty$ as $l \rightarrow \infty$. Thus, the smallness in $L^{\infty}$ holds whenever $l$ is suitably large. Note that the above estimate seems hard to be true for the non Maxwell molecule case. To treat this difficulty, motivated by [1], in case of hard potentials $0<\gamma \leq 1$, we instead make use of the following estimate:

$$
\sup _{|v| \geq M(l)}(1+|v|)^{-\gamma} w_{l}|\mathcal{K} f| \leq C\left\{(1+M(l))^{-\frac{\gamma}{2}}+\varsigma(l)\right\}\left\|w_{l} f\right\|_{L^{\infty}}
$$

for $C$ independent of $l$, where it holds that $M(l) \rightarrow \infty$ and $\varsigma(l) \rightarrow 0$ as $l \rightarrow \infty$. Then, the smallness in $L^{\infty}$ still holds when $l$ is chosen to be large enough. Therefore, in both cases $\gamma=0$ and $0<\gamma \leq 1$, the $L^{\infty}$ estimates combined with the $L^{2}$ estimates can be closed.

In addition, the coupled equations (1.19) and (1.20) will be solved by an iteration method in which the conservation laws $\left\langle G_{R, 1}+\mu^{\frac{1}{2}} G_{R, 2},\left[1, v_{i},|v|^{2}\right]\right\rangle=0(i=$ $1,2,3)$ play a crucial role. To ensure that the macroscopic moments of the iteration system are conserved, we design the following delicate approximation equations:

$$
\begin{aligned}
& \epsilon G_{R, 1}^{n+1}-\beta^{n} \nabla_{v} \cdot\left(v G_{R, 1}^{n+1}\right)-\alpha \nabla_{v} \cdot\left(A v G_{R, 1}^{n+1}\right)+v G_{R, 1}^{n+1}-\chi_{M} \mathcal{K} G_{R, 1}^{n+1}+\frac{\beta^{n}}{2}|v|^{2} \mu^{\frac{1}{2}} G_{R, 2}^{n+1} \\
& +\frac{1}{2} \alpha v \cdot(A v) \mu^{\frac{1}{2}} G_{R, 2}^{n+1}-\left(\beta_{1}^{n+1}-\frac{1}{3}\left\langle G_{1}, L G_{1}\right\rangle\right) \nabla_{v} \cdot(v \mu) \\
= & \frac{1}{3}\left\langle G_{1}, L G_{1}\right\rangle \nabla_{v} \cdot(v \mu)+\frac{\beta^{n}}{\alpha} \nabla_{v} \cdot\left(v \mu^{\frac{1}{2}} G_{1}\right)+\nabla_{v} \cdot\left(A v \sqrt{\mu} G_{1}\right)+Q\left(\mu^{\frac{1}{2}} G_{1}, \mu^{\frac{1}{2}} G_{1}\right)
\end{aligned}
$$




$$
\begin{aligned}
& \quad+\alpha\left\{Q\left(\mu^{\frac{1}{2}} G_{R}^{n}, \mu^{\frac{1}{2}} G_{1}\right)+Q\left(\mu^{\frac{1}{2}} G_{1}, \mu^{\frac{1}{2}} G_{R}^{n}\right)\right\}+\alpha^{2} Q\left(\mu^{\frac{1}{2}} G_{R}^{n}, \mu^{\frac{1}{2}} G_{R}^{n}\right), \\
& \epsilon G_{R, 2}^{n+1}-\beta^{n} \nabla_{v} \cdot\left(v G_{R, 2}^{n+1}\right)-\alpha \nabla_{v} \cdot\left(A v G_{R, 2}^{n+1}\right)+L G_{R, 2}^{n+1} \\
& \quad-\left(1-\chi_{M}\right) \mu^{-\frac{1}{2}} \mathcal{K} G_{R, 1}^{n+1}=0,
\end{aligned}
$$

where two penalty terms with the parameter $\epsilon>0$ have been added and

$$
\beta^{n}=-\frac{\alpha}{3} \operatorname{tr} A+\alpha^{2} \beta_{1}^{n}
$$

with

$$
\begin{aligned}
& \beta_{1}^{n}=\frac{1}{3} \int_{\mathbb{R}^{3}} G_{1} L G_{1} d v-\frac{\alpha}{3} \int_{\mathbb{R}^{3}} \mathbf{P}_{1}\{v \cdot(A v) \sqrt{\mu}\} G_{R}^{n} d v, \\
& \mu^{\frac{1}{2}} G_{R}^{n}=G_{R, 1}^{n}+\mu^{\frac{1}{2}} G_{R, 2}^{n} .
\end{aligned}
$$

System (1.21) provides us the following cancellations:

$$
\begin{gathered}
\left\langle\left(\beta_{1}^{n+1}-\frac{1}{3}\left\langle G_{1}, L G_{1}\right\rangle\right) \nabla_{v} \cdot(v \mu), \frac{1}{2}|v|^{2}\right\rangle-\frac{1}{2} \alpha\left\langle v \cdot(A v) \mu^{\frac{1}{2}} G_{R, 2}^{n+1}, \frac{1}{2}|v|^{2}\right\rangle \\
-\alpha\left\langle\nabla_{v} \cdot\left(A v G_{R, 1}^{n+1}\right), \frac{1}{2}|v|^{2}\right\rangle-\alpha\left\langle\nabla_{v} \cdot\left(A v G_{R, 2}^{n+1}\right), \frac{1}{2}|v|^{2} \sqrt{\mu}\right\rangle=0, \\
\frac{1}{3}\left\langle\left\langle G_{1}, L G_{1}\right\rangle \nabla_{v} \cdot(v \mu), \frac{1}{2}|v|^{2}\right\rangle+\left\langle\nabla_{v} \cdot\left(A v \sqrt{\mu} G_{1}\right), \frac{1}{2}|v|^{2}\right\rangle=0,
\end{gathered}
$$

which indeed give the energy conservation $\left\langle G_{R, 1}^{n+1}+\mu^{\frac{1}{2}} G_{R, 2}^{n+1},|v|^{2}\right\rangle=0$. Moreover, as in [16] for treating the nonlocal collision term, we introduce a $\sigma$-parameterized procedure to ensure the construction of solutions to the linear inhomogeneous system with $0 \leq \sigma \leq 1$; see Lemmas 2.1 and 2.2 for details. However, this induces the loss of conservation laws for the system with $0 \leq \sigma<1$ in the hard potential case $0<\gamma \leq 1$, which is quite different from the situation treated in [14].

The second point is concerned with the non-negativity of the steady profiles. For the purpose, we introduce a spatially inhomogeneous model (1.13) and prove the asymptotic stability of the stationary solution under small perturbation. We remark that although it is a spatially inhomogeneous problem, the proof with slight modifications can still be carried over to treat the spatially homogeneous case. One difficulty part is to obtain the macroscopic dissipation in a more delicate way than that in the steady case. In particular, we re-design the Caflisch's decomposition $\sqrt{\mu} f=f_{1}+\sqrt{\mu} f_{2}$ with $f_{1}$ and $f_{2}$ satisfying (3.3) and (3.5), respectively. The key point is to add a microscopic fourth-order moment function 
$\alpha A v \cdot\left(\nabla_{v} \sqrt{\mu}\right)\left(|v|^{2}-3\right) c_{f_{2}}$ to the left-hand of (3.5) in order to cancel such trouble term coming from $-\alpha \nabla_{v} \cdot\left(A v f_{2}\right)$. Correspondingly, (3.3) has been modified with $\alpha A v \cdot\left(\nabla_{v} \sqrt{\mu}\right)\left(|v|^{2}-3\right) \sqrt{\mu} c_{f_{2}}$ added to the right-hand side. Under such decomposition, the macroscopic energy $\|c\|^{2}$ and the microscopic energy $\left\|\mathbf{P}_{1} g_{2}\right\|^{2}$ can be combined for estimates, see the result (3.38). Thus, the corresponding energy dissipation rates $\alpha^{2}\|c\|^{2}$ and $\left\|\mathbf{P}_{1} g_{2}\right\|_{v}^{2}$ are obtained. This $L^{2}$ estimate (3.38) is crucial for obtaining the macroscopic dissipation and further deducing the exponential decay rate with the size proportional to $\alpha^{2}$. This size is the same as that in [14] for simple shear flow where $\operatorname{tr}(A)=0$ and the lowest order of $\beta$ is $\alpha^{2}$. In the current case for a general deformation matrix $A$, the lowest order of $\beta$ is $\alpha$ if $\operatorname{tr}(A) \neq 0$. We remark that it is unclear for us whether the degenerate order $\alpha^{2}$ for the size of decay rate is optimal. Moreover, similar to [2] for the study at the fluid level, it would be interesting to further consider possible enhanced decay rates with respect to any small $\alpha$ by using the deformation effect in case of the hard potentials $0<\gamma \leq 1$ and we will explore this issue in the future.

The third point is related to an application of the Guo's $L^{\infty}-L^{2}$ method [24]. The key idea of this approach in the $L^{\infty}$ estimate is to convert an integration with respect to $v$ variable along characteristics into an integration with respect to $x$ variable. In the process, one need obtain a proper control for the Jacobian

$$
\left|\frac{\partial X\left(s^{\prime}\right)}{\partial v_{*}}\right|=\left|(\beta I+\alpha A)^{-1}\left[e^{-\left(s^{\prime}-s\right)(\beta I+\alpha A)}-I\right]\right|
$$

along the following characteristic line:

$$
\begin{aligned}
& V\left(s^{\prime}\right)=V\left(s^{\prime} ; s, X(s), v_{*}\right)=e^{-\left(s^{\prime}-s\right)(\beta I+\alpha A)} v_{* \prime} \\
& X\left(s^{\prime}\right)=X\left(s^{\prime} ; s, X(s), v_{*}\right)=X(s)-(\beta I+\alpha A)^{-1}\left[e^{-\left(s^{\prime}-s\right)(\beta I+\alpha A)}-I\right] v_{*} .
\end{aligned}
$$

For this, as described in Lemma 4.8, we prove a lower bound of the determinant of a matrix exponential, and moreover, we also give an upper bound of the region of the integration after the change of variable $X\left(s^{\prime}\right) \rightarrow y$.

The rest of this paper is arranged as follows. The existence of the steady profile $G_{s t}(v)$ for (1.1) is established in Section 2. Section 3 is devoted to the unsteady problem (1.13) and (1.14). In Section 4 as an appendix, we give the basic estimates on the linearized operator $L$ as well as the nonlinear operators $\Gamma$ and $Q$, further present a key estimate for the operator $\mathcal{K}$ in the case of hard potentials, and finally derive a lower bound for a matrix exponential.

Notations. We give more notations to be used throughout the paper. Let $C$ denote some generic positive (generally large) constant and $\lambda$ denote some generic 
positive (generally small) constants, where $C$ and $\lambda$ may take different values in different places. Let $\mathbf{1}_{\mathcal{S}}$ be the characteristic function on the set $\mathcal{S}$. For simplicity, we use $\|\cdot\|$ to denote the norms of either $L^{2}\left(\mathbb{T}_{x}^{3} \times \mathbb{R}_{v}^{3}\right)$ or $L^{2}\left(\mathbb{T}_{x}^{3}\right)$ or $L^{2}\left(\mathbb{R}_{v}^{3}\right)$. We also use $\|\cdot\|_{L^{\infty}}$ to denote the norms of either $L^{\infty}\left(\mathbb{T}_{x}^{3} \times \mathbb{R}_{v}^{3}\right)$ or $L^{\infty}\left(\mathbb{R}_{v}^{3}\right)$. Moreover, $(\cdot, \cdot)$ denotes the inner product of $L^{2}\left(\mathbb{T}_{x}^{3} \times \mathbb{R}_{v}^{3}\right)$ and $\langle\cdot\rangle$ denotes the inner product of $L^{2}\left(\mathbb{R}_{v}^{3}\right)$.

\section{Steady problem}

This section is devoted to the existence of the non-equilibrium smooth steady solution of (1.1). We begin with some usual notations in the framework of perturbations around the global Maxwellian $\mu$ in (1.8). First of all, we introduce the linearized collision operator $L$ and the nonlinear collision operator $\Gamma$, defined by

$$
L g=-\mu^{-\frac{1}{2}}\{Q(\mu, \sqrt{\mu} g)+Q(\sqrt{\mu} g, \mu)\},
$$

and

$$
\begin{aligned}
& \Gamma(f, g)=\mu^{-\frac{1}{2}} Q(\sqrt{\mu} f, \sqrt{\mu} g) \\
= & \int_{\mathbb{R}^{3}} \int_{\mathrm{S}^{2}} B\left(\omega, v-v_{*}\right) \mu^{\frac{1}{2}}\left(v_{*}\right)\left[f\left(v_{*}^{\prime}\right) g\left(v^{\prime}\right)-f\left(v_{*}\right) g(v)\right] d \omega d v_{*},
\end{aligned}
$$

respectively. Note that

$$
L f=v f-K f
$$

with

$$
\begin{aligned}
& v=\int_{\mathbb{R}^{3}} \int_{\mathrm{S}^{2}} B\left(\omega, v-v_{*}\right) \mu\left(v_{*}\right) d \omega d v_{*} \backsim(1+|v|)^{\gamma}, \\
& K f=\mu^{-\frac{1}{2}}\left\{Q\left(\mu^{\frac{1}{2}} f, \mu\right)+Q_{\text {gain }}\left(\mu, \mu^{\frac{1}{2}} f\right)\right\},
\end{aligned}
$$

where $Q_{\text {gain }}$ denotes the positive part of $Q$ in (1.2). Moreover, it holds that

$$
K f=\int_{\mathbb{R}^{3}} \mathbf{k}\left(v, v_{*}\right) f\left(v_{*}\right) d v_{*}=\int_{\mathbb{R}^{3}}\left(\mathbf{k}_{2}-\mathbf{k}_{1}\right)\left(v, v_{*}\right) f\left(v_{*}\right) d v_{*}
$$

with

$$
\begin{aligned}
& 0 \leq \mathbf{k}_{1}\left(v, v_{*}\right) \leq \tilde{c}_{1}\left|v-v_{*}\right|^{\gamma} e^{-\frac{1}{4}\left(|v|^{2}+\left|v_{*}\right|^{2}\right)}, \\
& 0 \leq \mathbf{k}_{2}\left(v, v_{*}\right) \leq \tilde{c}_{2}\left|v-v_{*}\right|^{-2+\gamma} e^{-\frac{1}{8}\left|v-v_{*}\right|^{2}-\frac{1}{8} \frac{|v|^{2}-\left.\left|v_{*}\right|^{2}\right|^{2}}{\left|v-v_{*}\right|^{2}}},
\end{aligned}
$$


where both $\tilde{c}_{1}$ and $\tilde{c}_{2}$ are positive constants. Note that in the case of $\gamma=1,(2.4)$ has been derived in [21, pp.45-46], and the remaining cases that $\gamma \in[0,1)$ can be treated similarly. The upper bound in (2.4) may not be optimal.

The kernel of $L$, denoted as $\operatorname{ker} L$, is a five-dimensional space spanned by

$$
\left\{1, v,|v|^{2}-3\right\} \sqrt{\mu}:=\left\{\phi_{i}\right\}_{i=1}^{5} \text {. }
$$

We further define a projection from $L^{2}$ to $\operatorname{ker}(L)$ by

$$
\mathbf{P}_{0 g}=\left\{a_{g}+\mathbf{b}_{g} \cdot v+\left(|v|^{2}-3\right) c_{g}\right\} \sqrt{\mu}
$$

for $g \in L^{2}$, and correspondingly denote the operator $\mathbf{P}_{1}$ by $\mathbf{P}_{1} g=g-\mathbf{P}_{0} g$, which is orthogonal to $\mathbf{P}_{0}$ in $L^{2}$. Traditionally, $\mathbf{P}_{0} g$ is also called the macroscopic part, while $\mathbf{P}_{1} g$ stands for the microscopic component.

It is also convenient to define

$$
\mathcal{L} f=-\{Q(f, \mu)+Q(\mu, f)\}=v f-\mathcal{K} f
$$

with

$$
\mathcal{K} f=Q(f, \mu)+Q_{\text {gain }}(\mu, f)=\sqrt{\mu} K\left(\frac{f}{\sqrt{\mu}}\right)
$$

\subsection{Hilbert expansion and Caflisch's decomposition}

As derived before, we will study the steady problem

$$
-\beta \nabla_{v} \cdot\left(v G_{s t}\right)-\alpha \nabla_{v} \cdot\left(A v G_{s t}\right)=Q\left(G_{s t}, G_{s t}\right)
$$

with

$$
\beta=-\frac{\alpha}{3} \int_{\mathbb{R}^{3}} v \cdot(A v) G_{s t} d v
$$

Our goal is to look for a unique smooth solution $G_{s t}(v)$ satisfying

$$
\int_{\mathbb{R}^{3}} G_{s t} d v=1, \quad \int_{\mathbb{R}^{3}} v_{i} G_{s t} d v=0, \quad i=1,2,3, \quad \int_{\mathbb{R}^{3}}|v|^{2} G_{s t} d v=3 .
$$

Note that through the paper we have omitted the dependence of $G_{s t}$ on the parameter $\alpha$. It can be expected that $G_{s t} \rightarrow \mu$ if $\alpha \rightarrow 0$. As such, we set

$$
G_{s t}=\mu+\alpha \sqrt{\mu}\left\{G_{1}+\alpha G_{R}\right\}
$$


with $\mathbf{P}_{0} G_{1}=\mathbf{P}_{0} G_{R}=0$ such that (2.8) is valid, and hence we impose that

$$
\begin{aligned}
& \int_{\mathbb{R}^{3}} G_{1} \sqrt{\mu} d v=\int_{\mathbb{R}^{3}} G_{R} \sqrt{\mu} d v=0, \\
& \int_{\mathbb{R}^{3}} G_{1} v_{i} \sqrt{\mu} d v=\int_{\mathbb{R}^{3}} G_{R} v_{i} \sqrt{\mu} d v=0, \quad i=1,2,3, \\
& \int_{\mathbb{R}^{3}} G_{1}|v|^{2} \sqrt{\mu} d v=\int_{\mathbb{R}^{3}} G_{R}|v|^{2} \sqrt{\mu} d v=0,
\end{aligned}
$$

where $G_{1}$ is the first order correction and $G_{R}$ denotes the higher order remainder. Plugging (2.9) into (2.7), we get

$$
\beta=-\frac{\alpha}{3} \int_{\mathbb{R}^{3}} v \cdot(A v)\left[\mu+\alpha \sqrt{\mu}\left\{G_{1}+\alpha G_{R}\right\}\right] d v=\alpha \beta_{0}+\alpha^{2} \beta_{1}
$$

with

$$
\beta_{0}=-\frac{1}{3} \operatorname{tr} A, \beta_{1}=-\frac{1}{3} \int_{\mathbb{R}^{3}} v \cdot(A v)\left[\sqrt{\mu}\left\{G_{1}+\alpha G_{R}\right\}\right] d v .
$$

Furthermore, substituting (2.9) and (2.11) into (2.6) and comparing the coefficients in front of the different orders of $\alpha$, one has

$$
\begin{aligned}
& -\beta_{0} \mu^{-\frac{1}{2}} \nabla_{v} \cdot(v \mu)-\mu^{-\frac{1}{2}} \nabla_{v} \cdot(A v \mu)+L G_{1}=0, \\
- & \beta \mu^{-\frac{1}{2}} \nabla_{v} \cdot\left(v \sqrt{\mu} G_{R}\right)-\alpha \mu^{-\frac{1}{2}} \nabla_{v} \cdot\left(A v \sqrt{\mu} G_{R}\right)+L G_{R} \\
= & \beta_{1} \mu^{-\frac{1}{2}} \nabla_{v} \cdot(v \mu)+\frac{\beta}{\alpha} \mu^{-\frac{1}{2}} \nabla_{v} \cdot\left(v \sqrt{\mu} G_{1}\right)+\mu^{-\frac{1}{2}} \nabla_{v} \cdot\left(A v \sqrt{\mu} G_{1}\right) \\
& +\alpha\left\{\Gamma\left(G_{1}, G_{R}\right)+\Gamma\left(G_{R}, G_{1}\right)\right\}+\alpha^{2} \Gamma\left(G_{R}, G_{R}\right) .
\end{aligned}
$$

In light of expression for $\beta_{0}$ in (2.12), one gets from (2.13) that

$$
G_{1}=-\sum_{i, j=1}^{3} a_{i j} L^{-1}\left\{\left(v_{i} v_{j}-\frac{1}{3} \delta_{i j}|v|^{2}\right) \mu^{\frac{1}{2}}\right\},
$$

which in turn gives

$$
\begin{aligned}
\beta_{1}= & \frac{1}{3} \int_{\mathbb{R}^{3}} \mathbf{P}_{1}\{v \cdot(A v) \sqrt{\mu}\} L^{-1}\left\{\mathbf{P}_{1}\left(v \cdot A v \mu^{\frac{1}{2}}\right)\right\} d v \\
& \quad-\frac{\alpha}{3} \int_{\mathbb{R}^{3}} \mathbf{P}_{1}\{v \cdot(A v) \sqrt{\mu}\} G_{R} d v \\
= & \frac{1}{3} \int_{\mathbb{R}^{3}} G_{1} L G_{1} d v-\frac{\alpha}{3} \int_{\mathbb{R}^{3}} \mathbf{P}_{1}\{v \cdot(A v) \sqrt{\mu}\} G_{R} d v .
\end{aligned}
$$


Note that one has $\beta_{1}>0$ provided that $A$ is not a scalar matrix and $\alpha$ is suitably small.

The remainder $G_{R}$ is determined by (2.14). There is a severe growth term $\frac{\alpha}{2} v$. $(A v) G_{R}$ caused by the deformation force. To overcome this difficulty, as $[14,15]$, we resort to the following Caflisch's decomposition:

$$
\sqrt{\mu} G_{R}=G_{R, 1}+\sqrt{\mu} G_{R, 2}
$$

where $G_{R, 1}$ and $G_{R, 2}$ satisfy

$$
\begin{aligned}
- & \beta \nabla_{v} \cdot\left(v G_{R, 1}\right)-\alpha \nabla_{v} \cdot\left(A v G_{R, 1}\right)+v G_{R, 1} \\
=\chi & \chi_{M} \mathcal{K} G_{R, 1}-\frac{\beta}{2}|v|^{2} \sqrt{\mu} G_{R, 2}-\frac{\alpha}{2} v \cdot(A v) \sqrt{\mu} G_{R, 2}+\beta_{1} \nabla_{v} \cdot(v \mu) \\
& +\frac{\beta}{\alpha} \nabla_{v} \cdot\left(v \sqrt{\mu} G_{1}\right)+\nabla_{v} \cdot\left(A v \sqrt{\mu} G_{1}\right)+Q\left(\sqrt{\mu} G_{1}, \sqrt{\mu} G_{1}\right) \\
& +\alpha\left\{Q\left(\sqrt{\mu} G_{1}, \sqrt{\mu} G_{R}\right)+Q\left(\sqrt{\mu} G_{R}, \sqrt{\mu} G_{1}\right)\right\}+\alpha^{2} Q\left(\sqrt{\mu} G_{R}, \sqrt{\mu} G_{R}\right),
\end{aligned}
$$

and

$$
-\beta \nabla_{v} \cdot\left(v G_{R, 2}\right)-\alpha \nabla_{v} \cdot\left(A v G_{R, 2}\right)+L G_{R, 2}=\mu^{-\frac{1}{2}}\left(1-\chi_{M}\right) \mathcal{K} G_{R, 1},
$$

respectively. Here, $\chi_{M}(v)$ is a non-negative smooth cutoff function defined by

$$
\chi_{M}(v)= \begin{cases}1, & |v| \geq M+1 \\ 0, & |v| \leq M\end{cases}
$$

with $M>0$ sufficiently large.

We will prove the unique existence of (2.16) and (2.17) in the Banach space

$$
\begin{gathered}
\mathbf{X}_{m}=\left\{\mathcal{G}=\left[\mathcal{G}_{1}, \mathcal{G}_{2}\right] \mid \sum_{k \leq m}\left\{\left\|w_{l} \nabla_{v}^{k} \mathcal{G}_{1}\right\|_{L^{\infty}}+\left\|w_{l} \nabla_{v}^{k} \mathcal{G}_{2}\right\|_{L^{\infty}}\right\}<+\infty, k, m \in \mathbb{Z}^{+},\right. \\
\left.\left\langle\mathcal{G}_{1},\left[1, v_{i},|v|^{2}\right]\right\rangle+\left\langle\mathcal{G}_{2},\left[1, v_{i},|v|^{2}\right] \mu^{\frac{1}{2}}\right\rangle=0, i=1,2,3\right\}
\end{gathered}
$$

associated with the norm

$$
\left\|\left[\mathcal{G}_{1}, \mathcal{G}_{2}\right]\right\|_{\mathbf{X}, m}=\sum_{k \leq m}\left\{\left\|w_{l} \nabla_{v}^{k} \mathcal{G}_{1}\right\|_{L^{\infty}}+\left\|w_{l} \nabla_{v}^{k} \mathcal{G}_{2}\right\|_{L^{\infty}}\right\}
$$

To do so, we design the following iteration equations:

$$
\epsilon G_{R, 1}^{n+1}-\beta^{n} \nabla_{v} \cdot\left(v G_{R, 1}^{n+1}\right)-\alpha \nabla_{v} \cdot\left(A v G_{R, 1}^{n+1}\right)+v G_{R, 1}^{n+1}-\chi_{M} \mathcal{K} G_{R, 1}^{n+1}
$$




$$
\begin{aligned}
& \quad+\frac{\beta^{n}}{2}|v|^{2} \mu^{\frac{1}{2}} G_{R, 2}^{n+1}+\frac{1}{2} \alpha v \cdot(A v) \mu^{\frac{1}{2}} G_{R, 2}^{n+1}-\left(\beta_{1}^{n+1}-\frac{1}{3}\left\langle G_{1}, L G_{1}\right\rangle\right) \nabla_{v} \cdot(v \mu) \\
& =\frac{1}{3}\left\langle G_{1}, L G_{1}\right\rangle \nabla_{v} \cdot(v \mu)+\frac{\beta^{n}}{\alpha} \nabla_{v} \cdot\left(v \mu^{\frac{1}{2}} G_{1}\right)+\nabla_{v} \cdot\left(A v \sqrt{\mu} G_{1}\right) \\
& \quad+Q\left(\mu^{\frac{1}{2}} G_{1}, \mu^{\frac{1}{2}} G_{1}\right)+\alpha\left\{Q\left(\mu^{\frac{1}{2}} G_{R}^{n}, \mu^{\frac{1}{2}} G_{1}\right)+Q\left(\mu^{\frac{1}{2}} G_{1}, \mu^{\frac{1}{2}} G_{R}^{n}\right)\right\} \\
& \quad+\alpha^{2} Q\left(\mu^{\frac{1}{2}} G_{R}^{n}, \mu^{\frac{1}{2}} G_{R}^{n}\right) \\
& \epsilon G_{R, 2}^{n+1}-\beta^{n} \nabla_{v} \cdot\left(v G_{R, 2}^{n+1}\right)-\alpha \nabla_{v} \cdot\left(A v G_{R, 2}^{n+1}\right)+L G_{R, 2}^{n+1}-\left(1-\chi_{M}\right) \mu^{-\frac{1}{2}} \mathcal{K} G_{R, 1}^{n+1}=0
\end{aligned}
$$

Here the parameter $\epsilon>0$ is introduced such that all the conservation laws for $G_{R}^{n+1}$ as in (2.10) can be satisfied. Moreover, we have denoted

$$
\mu^{\frac{1}{2}} G_{R}^{n}=G_{R, 1}^{n}+\mu^{\frac{1}{2}} G_{R, 2}^{n}, \quad n \geq 0
$$

and

$$
\beta^{n}=\alpha \beta_{0}+\alpha^{2} \beta_{1}^{n}
$$

with

$$
\beta_{1}^{n}=\frac{1}{3} \int_{\mathbb{R}^{3}} G_{1} L G_{1} d v-\frac{\alpha}{3} \int_{\mathbb{R}^{3}} \mathbf{P}_{1}\{v \cdot(A v) \sqrt{\mu}\} G_{R}^{n} d v, \quad n \geq 0,
$$

as well as

$$
\left[G_{R, 1}^{0}, G_{R, 2}^{0}\right]=[0,0] .
$$

Note that the approximation solutions are constructed to satisfy (2.18), by which the following identities hold true:

$$
\begin{gathered}
\left\langle\left(\beta_{1}^{n+1}-\frac{1}{3}\left\langle G_{1}, L G_{1}\right\rangle\right) \nabla_{v} \cdot(v \mu), \frac{1}{2}|v|^{2}\right\rangle-\frac{1}{2} \alpha\left\langle v \cdot(A v) \mu^{\frac{1}{2}} G_{R, 2}^{n+1}, \frac{1}{2}|v|^{2}\right\rangle \\
-\alpha\left\langle\nabla_{v} \cdot\left(A v G_{R, 1}^{n+1}\right), \frac{1}{2}|v|^{2}\right\rangle-\alpha\left\langle\nabla_{v} \cdot\left(A v G_{R, 2}^{n+1}\right), \frac{1}{2}|v|^{2} \sqrt{\mu}\right\rangle=0, \\
\frac{1}{3}\left\langle\left\langle G_{1}, L G_{1}\right\rangle \nabla_{v} \cdot(v \mu), \frac{1}{2}|v|^{2}\right\rangle+\left\langle\nabla_{v} \cdot\left(A v \sqrt{\mu} G_{1}\right), \frac{1}{2}|v|^{2}\right\rangle=0,
\end{gathered}
$$

so that one can show the conservation laws (2.10) for $G_{R}^{n+1}$.

The proof of Theorem 1.1 consists of three steps. First, we show the wellposedness of the system (2.18) for given $\left[G_{R, 1}^{n}, G_{R, 2}^{n}\right]$ and $\epsilon>0$. Second, we establish the limit process $n \rightarrow+\infty$ for any fixed parameter $\epsilon>0$. Third, we pass the limits $\epsilon \rightarrow 0^{+}$to obtain the unique smooth solution of the system (2.16) and (2.17). 


\subsection{A uniform $L^{\infty}$ estimate with respect to the parameter $\sigma$}

Since both $K$ and $\mathcal{K}$ are nonlocal and do not possess the property of smallness, it is convenient to introduce the following linear vector operator parameterized by $\sigma \in[0,1]$ (cf. [14]):

$$
\begin{aligned}
& \mathscr{L}_{\sigma}\left[\mathcal{G}_{1}, \mathcal{G}_{2}\right]=\left[\mathscr{L}_{\sigma}^{1}, \mathscr{L}_{\sigma}^{2}\right]\left[\mathcal{G}_{1}, \mathcal{G}_{2}\right], \\
& \mathscr{L}_{\sigma}^{1}\left[\mathcal{G}_{1}, \mathcal{G}_{2}\right]=\epsilon \mathcal{G}_{1}-\beta^{\prime} \nabla_{v} \cdot\left(v \mathcal{G}_{1}\right)-\alpha \nabla_{v} \cdot\left(A v \mathcal{G}_{1}\right)+v \mathcal{G}_{1}-\sigma \chi_{M} \mathcal{K} \mathcal{G}_{1} \\
& \quad+\frac{\beta^{\prime}}{2}|v|^{2} \sqrt{\mu} \mathcal{G}_{2}+\alpha \frac{v \cdot(A v)}{2} \sqrt{\mu} \mathcal{G}_{2}-\beta^{\prime \prime}(\mathcal{G}) \nabla_{v} \cdot(v \mu), \\
& \mathscr{L}_{\sigma}^{2}\left[\mathcal{G}_{1}, \mathcal{G}_{2}\right]=\epsilon \mathcal{G}_{2}-\beta^{\prime} \nabla_{v} \cdot\left(v \mathcal{G}_{2}\right)-\alpha \nabla_{v} \cdot\left(A v \mathcal{G}_{2}\right)+v \mathcal{G}_{2}-\sigma K \mathcal{G}_{2} \\
& \quad-\sigma\left(1-\chi_{M}\right) \mu^{-\frac{1}{2}} \mathcal{K} \mathcal{G}_{1},
\end{aligned}
$$

where $\beta^{\prime}$ is a given constant of order $\alpha$, and

$$
\beta^{\prime \prime}(\mathcal{G})=-\frac{\alpha}{3} \int_{\mathbb{R}^{3}} \mathbf{P}_{1}\{v \cdot(A v)\}\left(\mathcal{G}_{1}+\sqrt{\mu} \mathcal{G}_{2}\right) d v .
$$

We then consider the solvability of the general coupled linear system

$$
\left\{\begin{array}{l}
\mathscr{L}_{\sigma}^{1}\left[\mathcal{G}_{1}, \mathcal{G}_{2}\right]=\mathcal{F}_{1} \\
\mathscr{L}_{\sigma}^{2}\left[\mathcal{G}_{1}, \mathcal{G}_{2}\right]=\mathcal{F}_{2}
\end{array}\right.
$$

where $\left[\mathcal{F}_{1}, \mathcal{F}_{2}\right]$ is given.

Remark 2.1. Note that in the case of $0<\gamma \leq 1$ (hard potentials) and $\sigma \neq 1$, the approximation system (2.21) does not imply $\left[\mathcal{G}_{1}, \mathcal{G}_{2}\right] \in \mathbf{X}_{m}$ even if $\left[\mathcal{F}_{1}, \mathcal{F}_{2}\right] \in \mathbf{X}_{m}$, because the structural damage of the linear operators $\mathcal{L}$ and $L$ violates the following laws of conservation:

$$
\left\langle\mathcal{G}_{1},\left[1, v_{i},|v|^{2}\right]\right\rangle+\left\langle\mathcal{G}_{2},\left[1, v_{i},|v|^{2}\right] \mu^{\frac{1}{2}}\right\rangle=0, \quad i=1,2,3 .
$$

Due to the above remark, different from [14] in the pure Maxwell molecule case, a convenient functional space to be considered is the following:

$$
\overline{\mathbf{X}}_{m}=\left\{\mathcal{G}=\left[\mathcal{G}_{1}, \mathcal{G}_{2}\right] \mid \sum_{k \leq m}\left\{\left\|w_{l} \nabla_{v}^{k} \mathcal{G}_{1}\right\|_{L^{\infty}}+\left\|w_{l} \nabla_{v}^{k} \mathcal{G}_{2}\right\|_{L^{\infty}}\right\}<+\infty, k, m \in \mathbb{Z}^{+}\right\}
$$

equipped with the norm

$$
\left\|\left[\mathcal{G}_{1}, \mathcal{G}_{2}\right]\right\|_{\overline{\mathbf{x}}_{m}}=\sum_{k \leq m}\left\{\left\|w_{l} \nabla_{v}^{k} \mathcal{G}_{1}\right\|_{L^{\infty}}+\left\|w_{l} \nabla_{v}^{k} \mathcal{G}_{2}\right\|_{L^{\infty}}\right\}
$$

The main idea showing the well-posedness of (2.21) is to adopt the bootstrap argument based on the following a priori $L^{\infty}$ estimates. 
Lemma 2.1 (A Priory Estimate). Assume that $\beta^{\prime} \neq 0$ is of order $\alpha$. Let $\left[\mathcal{G}_{1}, \mathcal{G}_{2}\right] \in \overline{\mathbf{X}}_{m}$ with $m \geq 0$ be a solution to (2.21) with $\epsilon>0$ and suitably small, $\sigma \in[0,1)$ and $\left[\mathcal{F}_{1}, \mathcal{F}_{2}\right] \in \overline{\mathbf{X}}_{m}$. There is $l_{0}>0$ such that for any $l \geq l_{0}$ arbitrarily large, there are $\alpha_{0}=\alpha_{0}(l)>0$ and large $M=M(l)>0$ such that for any $0<\alpha<\alpha_{0}$ with $C \alpha<1-\sigma$ for a generic large constant $C>0$, the solution $\left[\mathcal{G}_{1}, \mathcal{G}_{2}\right]$ of the system (2.21) satisfies the following estimate:

$$
\begin{gathered}
\left\|\left[\mathcal{G}_{1}, \mathcal{G}_{2}\right]\right\|_{\overline{\mathbf{x}}_{m}}=\left\|\mathscr{L}_{\sigma}^{-1}\left[\mathcal{F}_{1}, \mathcal{F}_{2}\right]\right\|_{\overline{\mathbf{x}}_{m}} \\
\leq C_{\mathscr{L}} \sum_{0 \leq k \leq m}\left\{\left\|w_{l} \nabla_{v}^{k} \mathcal{F}_{1}\right\|_{L^{\infty}}+\left\|w_{l} \nabla_{v}^{k} \mathcal{F}_{2}\right\|_{L^{\infty}}\right\},
\end{gathered}
$$

where the constant $C_{\mathscr{L}}>0$ depends on $\epsilon$ but not on $\sigma$ and $\alpha$.

Proof. The proof is divided into two steps.

Step 1. $L^{\infty}$ estimates. Taking $0 \leq k \leq m$ and $l>0$, we set $H_{1, k}=w_{l} \nabla_{v}^{k} \mathcal{G}_{1}$ and $H_{2, k}=$ $w_{l} \nabla_{v}^{k} \mathcal{G}_{2}$. Then, $\mathbf{H}_{k}=\left[H_{1, k}, H_{2, k}\right]$ satisfies the following equations:

$$
\begin{aligned}
& \epsilon H_{1, k}-\beta^{\prime} \nabla_{v} \cdot\left(v H_{1, k}\right)+2 l \beta^{\prime} \frac{|v|^{2}}{1+|v|^{2}} H_{1, k}-\alpha \nabla_{v} \cdot\left(A v H_{1, k}\right) \\
& \quad+2 l \alpha \frac{v \cdot(A v)}{1+|v|^{2}} H_{1, k}+v H_{1, k}-\sigma \chi_{M} w_{l} \mathcal{K}\left(\frac{H_{1, k}}{w_{l}}\right)-w_{l} \beta^{\prime \prime}\left(\frac{\mathbf{H}_{0}}{w_{l}}\right) \nabla_{v}^{k} \nabla_{v} \cdot(v \mu) \\
& =\mathbf{1}_{k^{\prime}=1} w_{l} \beta^{\prime} C_{k}^{k^{\prime}} \nabla_{v} \cdot\left(\nabla_{v}^{k^{\prime}} v \nabla_{v}^{k-k^{\prime}} \mathcal{G}_{1}\right)+\mathbf{1}_{k^{\prime}=1} \alpha C_{k}^{k^{\prime}} w_{l} \nabla_{v} \cdot\left(\nabla_{v}^{k^{\prime}}(A v) \nabla_{v}^{k-k^{\prime}} \mathcal{G}_{1}\right) \\
& \quad-\frac{\beta^{\prime}}{2} w_{l} \sum_{k^{\prime} \leq k} C_{k}^{k^{\prime}} \nabla_{v}^{k^{\prime}}\left(|v|^{2} \mu^{\frac{1}{2}}\right) \nabla_{v}^{k-k^{\prime}} \mathcal{G}_{2}-\frac{\alpha}{2} \sum_{k^{\prime} \leq k} w_{l} C_{k}^{k^{\prime}} \nabla_{v}^{k^{\prime}}\left(v \cdot(A v) \mu^{\frac{1}{2}}\right) \nabla_{v}^{k-k^{\prime}} \mathcal{G}_{2} \\
& \quad-\mathbf{1}_{k>0} w_{l} \sum_{0<k^{\prime} \leq k} C_{k}^{k^{\prime}} \nabla_{v}^{k^{\prime}} v \nabla_{v}^{k-k^{\prime}} \mathcal{G}_{1}+\mathbf{1}_{k>0} \sigma \sum_{0<k^{\prime} \leq k} C_{k}^{k^{\prime}} w_{l} \nabla_{v}^{k^{\prime}}\left(\chi_{M} \mathcal{K}\right) \nabla_{v}^{k-k^{\prime}} \mathcal{G}_{1}+w_{l} \nabla_{v}^{k} \mathcal{F}_{1}, \\
& \epsilon H_{2, k}-\beta^{\prime} \nabla_{v} \cdot\left(v H_{2, k}\right)+2 l \beta^{\prime} \frac{|v|^{2}}{1+|v|^{2}} H_{2, k}-\alpha \nabla_{v} \cdot\left(A v H_{2, k}\right) \\
& +2 l \alpha \frac{v \cdot(A v)}{1+|v|^{2}} H_{2, k}+v H_{2, k}-\sigma w_{l} K\left(\frac{H_{2, k}}{w_{l}}\right) \\
& =\mathbf{1}_{k^{\prime}=1} w_{l} \beta^{\prime} C_{k}^{k^{\prime}} \nabla_{v} \cdot\left(\nabla_{v}^{k^{\prime}} \nabla_{v}^{k-k^{\prime}} \mathcal{G}_{2}\right)+\mathbf{1}_{k^{\prime}=1} \alpha C_{k}^{k^{\prime}} w_{l} \nabla_{v} \cdot\left(\nabla_{v}^{k^{\prime}}(A v) \nabla_{v}^{k-k^{\prime}} \mathcal{G}_{2}\right) \\
& \quad-\mathbf{1}_{k>0} w_{l} \sum_{0<k^{\prime} \leq k} C_{k}^{k^{\prime}} \nabla_{v}^{k^{\prime}} v \nabla_{v}^{k-k^{\prime}} \mathcal{G}_{2}+\mathbf{1}_{k>0} \sigma w_{l} \sum_{0<k^{\prime} \leq k} C_{k}^{k^{\prime}} \nabla_{v}^{k^{\prime}} K \nabla_{v}^{k-k^{\prime}} \mathcal{G}_{2} \\
& \quad+\sigma \sum_{k^{\prime} \leq k} C_{k}^{k^{\prime}} w_{l} \nabla_{v}^{k^{\prime}}\left(\left(1-\chi_{M}\right) \mu^{-\frac{1}{2}} \mathcal{K}\right) \nabla_{v}^{k-k^{\prime}} \mathcal{G}_{1}+w_{l} \nabla_{v}^{k} \mathcal{F}_{2},
\end{aligned}
$$

where

$$
\mathbf{H}_{0}:=\left[H_{1}, H_{2}\right]=\left[H_{1,0}, H_{2,0}\right]=w_{l}\left[\mathcal{G}_{1}, \mathcal{G}_{2}\right] .
$$


Notice that (2.23) and (2.24) are linear PDEs of first order, it is convenient to apply the method of characteristics to obtain $L^{\infty}$ estimate (cf. $\left.[17,18]\right)$. To do this, we first introduce a uniform parameter $t \in \mathbb{R}$, and regard $H_{i, k}(v)=H_{i, k}(t, v)(i=1,2)$, then define the characteristic line $[s, V(s ; t, v)]$ for Eqs. (2.23) and (2.24) going through $(t, v)$ such that

$$
\begin{aligned}
& \frac{d V}{d s}=-\beta^{\prime} V(s ; t, v)-\alpha A V(s ; t, v), \\
& V(t ; t, v)=v
\end{aligned}
$$

which is equivalent to

$$
V(s)=V(s ; t, v)=e^{-(s-t)\left(\beta^{\prime} I+\alpha A\right)} v .
$$

Since $\beta^{\prime} \neq 0$, it is natural to expect that $|V(s)| \rightarrow+\infty$ as $s \rightarrow-\infty$ and $G_{R}(v) \rightarrow 0$ as $|v| \rightarrow+\infty$. Due to this, integrating along the backward trajectory (2.25) with respect to $s \in(-\infty, t]$, one can write the solutions of (2.23) and (2.24) as the mild form of

$$
H_{1, k}(v(t))=\sum_{i=1}^{6} \mathcal{I}_{i}
$$

with

$$
\begin{aligned}
& \mathcal{I}_{1}=\sigma \int_{-\infty}^{t} e^{-\int_{s}^{t} \mathcal{A}^{\epsilon}(\tau, V(\tau)) d \tau}\left\{\chi_{M} w_{l} \mathcal{K}\left(\frac{H_{1, k}}{w_{l}}\right)\right\}(V(s)) d s, \\
& \mathcal{I}_{2}=\int_{-\infty}^{t} e^{-\int_{s}^{t} \mathcal{A}^{\epsilon}(\tau, V(\tau)) d \tau}\left\{w_{l} \beta^{\prime \prime}\left(\frac{\mathbf{H}_{0}}{w_{l}}\right) \nabla_{v}^{k} \nabla_{v} \cdot(v \mu)\right\}(V(s)) d s, \\
& \mathcal{I}_{3}=\int_{-\infty}^{t} e^{-\int_{s}^{t} \mathcal{A}^{\epsilon}(\tau, V(\tau)) d \tau}\left\{\mathbf{1}_{k^{\prime}=1} w_{l} \beta^{\prime} C_{k}^{k^{\prime}} \nabla_{v} \cdot\left(\nabla_{v}^{k^{\prime}} v \nabla_{v}^{k-k^{\prime}} \mathcal{G}_{1}\right)\right. \\
& \left.+\mathbf{1}_{k^{\prime}=1} \alpha C_{k}^{k^{\prime}} w_{l} \nabla_{v} \cdot\left(\nabla_{v}^{k^{\prime}}(A v) \nabla_{v}^{k-k^{\prime}} \mathcal{G}_{1}\right)\right\}(V(s)) d s, \\
& \mathcal{I}_{4}=-\int_{-\infty}^{t} e^{-\int_{s}^{t} \mathcal{A}^{\epsilon}(\tau, V(\tau)) d \tau} \sum_{k^{\prime} \leq k} C_{k}^{k^{\prime}}\left\{\frac{\beta^{\prime}}{2} w_{l} \nabla_{v}^{k^{\prime}}\left(|v|^{2} \mu^{\frac{1}{2}}\right) \nabla_{v}^{k-k^{\prime}} \mathcal{G}_{2}\right. \\
& \left.+\frac{\alpha}{2} w_{l} \nabla_{v}^{k^{\prime}}\left(v \cdot(A v) \mu^{\frac{1}{2}}\right) \nabla_{v}^{k-k^{\prime}} \mathcal{G}_{2}\right\}(V(s)) d s, \\
& \mathcal{I}_{5}=\mathbf{1}_{k>0} \sigma \int_{-\infty}^{t} e^{-\int_{s}^{t} \mathcal{A}^{\epsilon}(\tau, V(\tau)) d \tau} \sum_{0<k^{\prime} \leq k} C_{k}^{k^{\prime}}\left\{-w_{l} \nabla_{v}^{k^{\prime}} \nu \nabla_{v}^{k-k^{\prime}} \mathcal{G}_{1}\right. \\
& \left.+w_{l} \nabla_{v}^{k^{\prime}}\left(\chi_{M} \mathcal{K}\right) \nabla_{v}^{k-k^{\prime}} \mathcal{G}_{1}\right\}(V(s)) d s,
\end{aligned}
$$




$$
\mathcal{I}_{6}=\int_{-\infty}^{t} e^{-\int_{s}^{t} \mathcal{A}^{\epsilon}(\tau, V(\tau)) d \tau}\left(w_{l} \nabla_{v}^{k} \mathcal{F}_{1}\right)(V(s)) d s
$$

and

$$
H_{2, k}=\sum_{i=7}^{11} \mathcal{I}_{i}
$$

with

$$
\begin{aligned}
\mathcal{I}_{7}= & \sigma \int_{-\infty}^{t} e^{-\int_{s}^{t} \mathcal{A}^{\epsilon}(\tau, V(\tau)) d \tau}\left[w_{l} K\left(\frac{H_{2, k}}{w_{l}}\right)\right](V(s)) d s, \\
\mathcal{I}_{8}= & \int_{-\infty}^{t} e^{-\int_{s}^{t} \mathcal{A}^{\epsilon}(\tau, V(\tau)) d \tau}\left\{\mathbf{1}_{k^{\prime}=1} w_{l} \beta^{\prime} C_{k}^{k^{\prime}} \nabla_{v} \cdot\left(\nabla_{v}^{k^{\prime}} v \nabla_{v}^{k-k^{\prime}} \mathcal{G}_{2}\right)\right. \\
& \left.\quad+\mathbf{1}_{k^{\prime}=1} \alpha C_{k}^{k^{\prime}} w_{l} \nabla_{v} \cdot\left(\nabla_{v}^{k^{\prime}}(A v) \nabla_{v}^{k-k^{\prime}} \mathcal{G}_{2}\right)\right\}(V(s)) d s, \\
\mathcal{I}_{9}= & \sigma \mathbf{1}_{k>0} \int_{-\infty}^{t} e^{-\int_{s}^{t} \mathcal{A}^{\epsilon}(\tau, V(\tau)) d \tau} \\
& \times \sum_{0<k^{\prime} \leq k} C_{k}^{k^{\prime}}\left\{-w_{l} \nabla_{v}^{k^{\prime}} \nu \nabla_{v}^{k-k^{\prime}} \mathcal{G}_{2}+w_{l} \nabla_{v}^{k^{\prime}} K \nabla_{v}^{k-k^{\prime}} \mathcal{G}_{2}\right\}(V(s)) d s, \\
\mathcal{I}_{10}= & \sigma \int_{-\infty}^{t} e^{-\int_{s}^{t} \mathcal{A}^{\epsilon}(\tau, V(\tau)) d \tau} \\
& \times\left\{\sum_{k^{\prime} \leq k} C_{k}^{k^{\prime}} w_{l} \nabla_{v}^{k^{\prime}}\left(\left(1-\chi_{M}\right) \mu^{-\frac{1}{2}} \mathcal{K}\right)\left(\nabla_{v}^{k-k^{\prime}} \mathcal{G}_{1}\right)\right\}(V(s)) d s, \\
\mathcal{I}_{11}= & \int_{-\infty}^{t} e^{-\int_{s}^{t} \mathcal{A}^{\epsilon}(\tau, V(\tau)) d \tau}\left(w_{l} \nabla_{v}^{k} \mathcal{F}_{2}\right)(V(s)) d s,
\end{aligned}
$$

where

$$
\begin{aligned}
& \mathcal{A}^{\epsilon}(\tau, V(\tau)) \\
= & v(V(\tau))+\epsilon-3 \beta^{\prime}+2 l \beta^{\prime} \frac{|V(\tau)|^{2}}{1+|V(\tau)|^{2}}+2 l \alpha \frac{V(\tau) \cdot(A V(\tau))}{1+|V(\tau)|^{2}}-\alpha \operatorname{tr} A \\
\geq & \frac{1}{2} v(V(\tau)),
\end{aligned}
$$

provided that $\epsilon>0, \alpha>0, l \alpha$ and $|\alpha \operatorname{tr} A|$ are suitably small. Note that $\nu(V(\tau))$ is independent of $V(\tau)$ in the Maxwell molecule case. Here and in the sequel, the velocity derivatives $\nabla_{v}^{k^{\prime}}$ acting on the nonlocal operators such as $\mathcal{K}, K$, etc. are understood in the way as (4.2). 
In what follows, we will compute $\mathcal{I}_{i}(1 \leq i \leq 11)$, separately. The estimates for $\mathcal{I}_{1}$ is divided into two cases. If $\gamma=0$ i.e. the Maxwell molecule case, we apply (4.11) in Lemma 4.4 to obtain that

$$
\mathcal{I}_{1} \leq \frac{C}{l}\left\|H_{1, k}(v)\right\|_{L^{\infty}} \int_{-\infty}^{t} e^{-\frac{v_{0}}{2}(t-s)} d s \leq \frac{C}{l}\left\|H_{1, k}(v)\right\|_{L^{\infty}},
$$

where

$$
v_{0}=\int_{\mathbb{R}^{3}} \int_{\mathbb{S}^{2}} B_{0}(\cos \theta) \mu\left(v_{*}\right) d \omega d v_{*}>0 .
$$

If $0<\gamma \leq 1$, Lemma 4.6 leads us to

$$
\begin{aligned}
\mathcal{I}_{1} & \leq \int_{-\infty}^{t} e^{-\int_{s}^{t} v(V(\tau)) d \tau} v(V(s))[v(V(s))]^{-1}\left\{\chi_{M} w_{l} \mathcal{K}\left(\frac{H_{1, k}}{w_{l}}\right)\right\}(V(s)) d s \\
& \leq \int_{-\infty}^{t} e^{-\int_{s}^{t} v(V(\tau)) d \tau} v(V(s))\left(\frac{C}{(1+M)^{\frac{\gamma}{2}}}+\varsigma\right)\left\|H_{1, k}(v)\right\|_{L^{\infty}} d s \\
& \leq\left(\frac{C}{(1+M)^{\frac{\gamma}{2}}}+\varsigma\right)\left\|H_{1, k}(v)\right\|_{L^{\infty}}
\end{aligned}
$$

where the following estimate has been used:

$$
\int_{-\infty}^{t} e^{-\int_{s}^{t} v(V(\tau)) d \tau} v(V(s)) d s \leq 1 .
$$

By virtue of (2.20), one has

$$
\mathcal{I}_{2} \leq C \alpha\left\|H_{1,0}\right\|_{L^{\infty}}+C \alpha\left\|H_{2,0}\right\|_{L^{\infty}} .
$$

It is straightforward to see that

$$
\mathcal{I}_{3} \leq C \alpha \sum_{k^{\prime} \leq k}\left\|H_{1, k^{\prime}}\right\|_{L^{\infty}}, \quad \mathcal{I}_{4}, \mathcal{I}_{8} \leq C \alpha \sum_{k^{\prime} \leq k}\left\|H_{2, k^{\prime}}\right\|_{L^{\infty}} .
$$

For $\mathcal{I}_{5}$, we first rewrite $\nabla_{v}^{k^{\prime}}\left(\chi_{M} \mathcal{K}\right)\left(\nabla_{v}^{k-k^{\prime}} \mathcal{G}_{1}\right)$ as

$$
\begin{aligned}
& \nabla_{v}^{k^{\prime}}\left(\chi_{M} \mathcal{K}\right)\left(\nabla_{v}^{k-k^{\prime}} \mathcal{G}_{1}\right) \\
= & \sum_{k^{\prime \prime} \leq k^{\prime}} C_{k^{\prime}}^{k^{\prime \prime}} \nabla_{v}^{k^{\prime}-k^{\prime \prime}} \chi_{M} \nabla_{v}^{k^{\prime \prime}} \mathcal{K}\left(\nabla_{v}^{k-k^{\prime}} \mathcal{G}_{1}\right) \\
= & \sum_{k^{\prime \prime} \leq k^{\prime}} C_{k^{\prime}}^{k^{\prime \prime}} \nabla_{v}^{k^{\prime}-k^{\prime \prime}} \chi_{M} \nabla_{v}^{k^{\prime \prime}}\left\{Q\left(\mu, \nabla_{v}^{k-k^{\prime}} \mathcal{G}_{1}\right)+Q\left(\nabla_{v}^{k-k^{\prime}} \mathcal{G}_{1}, \mu\right)\right\} .
\end{aligned}
$$


Then it follows that

$$
\mathcal{I}_{5} \mathbf{1}_{k \geq 1} \leq C \sum_{k^{\prime}<k}\left\|H_{1, k^{\prime}}\right\|_{L^{\infty}},
$$

according to Lemma 4.7. And likewise, we also have

$$
\mathcal{I}_{10} \leq C \sum_{k^{\prime} \leq k}\left\|H_{1, k^{\prime}}\right\|_{L^{\infty}}
$$

Next, Lemma 4.2 leads us to have

$$
\mathcal{I}_{9} \mathbf{1}_{k \geq 1} \leq C \sum_{k^{\prime}<k}\left\|H_{2, k^{\prime}}\right\|_{L^{\infty}}
$$

For $\mathcal{I}_{6}$ and $\mathcal{I}_{11}$, one directly has

$$
\mathcal{I}_{6} \leq C\left\|w_{l} \nabla_{v}^{k} \mathcal{F}_{1}\right\|_{L^{\infty}}, \quad \mathcal{I}_{11} \leq C\left\|w_{l} \nabla_{v}^{k} \mathcal{F}_{2}\right\|_{L^{\infty}}
$$

Finally, for the delicate term $\mathcal{I}_{7}$, we divide our computations into the following three cases.

Case 1. $|V| \geq M$ with $M$ suitably large. From Lemma 4.1, it follows that

$$
\int \mathbf{k}_{w}\left(V, v_{*}\right) d v_{*} \leq \frac{C}{1+|V|} \leq \frac{C}{M}
$$

Applying this, one has

$$
\mathcal{I}_{7} \leq \sup _{-\infty<s \leq t} \int_{\mathbb{R}^{3}} \mathbf{k}_{w}\left(V, v_{*}\right) d v_{*}\left\|H_{2, k}\right\|_{L^{\infty}} \leq \frac{C}{M}\left\|H_{2, k}\right\|_{L^{\infty}} .
$$

Case 2. $|V| \leq M$ and $\left|v_{*}\right| \geq 2 M$. In this situation, we have $\left|V-v_{*}\right| \geq M$, then

$$
\mathbf{k}_{w}\left(V, v_{*}\right) \leq C e^{-\frac{\varepsilon M^{2}}{8}} \mathbf{k}_{w}\left(V, v_{*}\right) e^{\frac{\varepsilon\left|V-v_{*}\right|^{2}}{8}} .
$$

Using Lemma 4.1, one sees that $\int \mathbf{k}_{w}\left(V, v_{*}\right) e^{\frac{\varepsilon\left|V-v_{*}\right|^{2}}{8}} d v_{*}$ is still bounded. Therefore, by a similar argument as for obtaining (2.26), it follows that

$$
\mathcal{I}_{7} \leq C e^{-\frac{\varepsilon M^{2}}{8}}\left\|H_{2, k}\right\|_{L^{\infty}}
$$

To complete our estimates for $\mathcal{I}_{7}$, we are now in a position to handle the last case. 
Case 3. $|V| \leq M$ and $\left|v_{*}\right| \leq 2 M$. In this case, the key point is to convert the bound in $L^{\infty}$-norm to the one in $L^{2}$-norm which will be established later on. To do so, for any large $M>0$, we choose a number $p=p(M)$ to define

$$
\mathbf{k}_{w, p}\left(V, v_{*}\right) \equiv \mathbf{1}_{\left|V-v_{*}\right| \geq \frac{1}{p},\left|v_{*}\right| \leq p} \mathbf{k}_{w}\left(V, v_{*}\right),
$$

such that

$$
\sup _{V} \int_{\mathbf{R}^{3}}\left|\mathbf{k}_{w, p}\left(V, v_{*}\right)-\mathbf{k}_{w}\left(V, v_{*}\right)\right| d v_{*} \leq \frac{1}{M} .
$$

One then has

$$
\begin{aligned}
\mathcal{I}_{7} & \leq C \sup _{s} \int_{\left|v_{*}\right| \leq 2 M} \mathbf{k}_{w, p}\left(V, v_{*}\right)\left|\nabla_{v}^{k} \mathcal{G}_{2}\left(v_{*}\right)\right| d v_{*}+\frac{1}{M}\left\|H_{2, k}\right\|_{L^{\infty}} \\
& \leq C(p) \sup _{s}\left\|\nabla_{v}^{k} \mathcal{G}_{2}\right\|+\frac{1}{M}\left\|H_{2, k}\right\|_{L^{\infty}},
\end{aligned}
$$

according to Hölder's inequality and the fact that

$$
\int_{\mathbb{R}^{3}} \mathbf{k}_{w, p}^{2}\left(V, v_{*}\right) d v_{*}<\infty .
$$

Therefore, it follows that for any large $M>0$,

$$
\mathcal{I}_{7} \leq C\left(e^{-\frac{\varepsilon M^{2}}{8}}+\frac{1}{M}\right)\left\|H_{2, k}\right\|_{L^{\infty}}+C\left\|\nabla_{v}^{k} \mathcal{G}_{2}\right\| .
$$

Combing all the estimates above together, we now arrive at

$$
\begin{aligned}
& \left\|H_{1, k}\right\|_{L^{\infty}} \leq\left(\mathbf{1}_{0<\gamma \leq 1} \frac{C}{(1+M)^{\frac{\gamma}{2}}}+\varsigma+\frac{C}{l}+C \alpha\right)\left\|H_{1, k}\right\|_{L^{\infty}}+C \alpha\left\|H_{1,0}\right\|_{L^{\infty}} \\
& +C \alpha \sum_{k^{\prime} \leq k}\left\|H_{2, k^{\prime}}\right\|_{L^{\infty}}+\mathbf{1}_{k \geq 1} C \sum_{k^{\prime}<k}\left\|H_{1, k^{\prime}}\right\|_{L^{\infty}}+C\left\|w_{l} \nabla_{v}^{k} \mathcal{F}_{1}\right\|_{L^{\infty}} \\
& \left\|H_{2, k}\right\|_{L^{\infty}} \leq\left(e^{-\frac{\varepsilon M^{2}}{8}}+\frac{C}{M}+C \alpha\right)\left\|H_{2, k}\right\|_{L^{\infty}}+\mathbf{1}_{k \geq 1} C \sum_{k^{\prime}<k}\left\|H_{2, k^{\prime}}\right\|_{L^{\infty}} \\
& +C \sum_{k^{\prime} \leq k}\left\|H_{1, k^{\prime}}\right\|_{L^{\infty}}+C\left\|\nabla_{v}^{k} \mathcal{G}_{2}\right\|+C\left\|w_{l} \nabla_{v}^{k} \mathcal{F}_{2}\right\|_{L^{\infty}} .
\end{aligned}
$$

It should be pointed out that the constant $C$ in (2.28) is independent of $\sigma$ and $\epsilon$. 
Step 2. $L^{2}$ estimates. To close our estimates, we now turn to deduce the $H^{k}$ estimate on $\mathcal{G}_{2}$. To do this, we start from the basic $L^{2}$ estimate of $\mathcal{G}_{2}$. By the inner product $\left\langle(2.21 \mathrm{~b}), \mathcal{G}_{2}\right\rangle$, one has

$$
\begin{aligned}
\epsilon & \left\langle\mathcal{G}_{2}, \mathcal{G}_{2}\right\rangle-\beta^{\prime}\left\langle\nabla_{v} \cdot\left(v \mathcal{G}_{2}\right), \mathcal{G}_{2}\right\rangle-\alpha\left\langle\nabla_{v} \cdot\left(A v \mathcal{G}_{2}\right), \mathcal{G}_{2}\right\rangle \\
& +(1-\sigma)\left\langle\nu \mathcal{G}_{2}, \mathcal{G}_{2}\right\rangle+\sigma\left\langle L \mathcal{G}_{2}, \mathcal{G}_{2}\right\rangle-\sigma\left\langle\left(1-\chi_{M}\right) \mu^{-\frac{1}{2}} \mathcal{K G}_{\mathcal{G}_{1}}, \mathcal{G}_{2}\right\rangle=\left\langle\mathcal{F}_{2}, \mathcal{G}_{2}\right\rangle,
\end{aligned}
$$

where we have used the identity

$$
v f-\sigma K f=(1-\sigma) v f+\sigma L f .
$$

Applying Lemma 4.3 and Cauchy-Schwarz's inequality, we get from (2.29) that for $l>\frac{3}{2}$

$$
\begin{aligned}
& \epsilon\left\|\mathcal{G}_{2}\right\|^{2}+(1-\sigma)\left\|\mathcal{G}_{2}\right\|_{v}^{2}+\delta_{0} \sigma\left\|\mathbf{P}_{1} \mathcal{G}_{2}\right\|_{v}^{2} \\
\leq & C \alpha\left\|\mathcal{G}_{2}\right\|^{2}+\frac{\epsilon}{4}\left\|\mathcal{G}_{2}\right\|^{2}+\frac{C}{\epsilon}\left\|w_{l} \mathcal{G}_{1}\right\|_{L^{\infty}}^{2}+\frac{C}{\epsilon}\left\|w_{l} \mathcal{F}_{2}\right\|_{L^{\infty}}^{2}
\end{aligned}
$$

which further implies

$$
\frac{\epsilon}{2}\left\|\mathcal{G}_{2}\right\|^{2}+(1-\sigma)\left\|\mathcal{G}_{2}\right\|_{v}^{2}+\delta_{0} \sigma\left\|\mathbf{P}_{1} \mathcal{G}_{2}\right\|_{v}^{2} \leq \frac{C}{\epsilon}\left\|w_{l} \mathcal{G}_{1}\right\|_{L^{\infty}}^{2}+\frac{C}{\epsilon}\left\|w_{l} \mathcal{F}_{2}\right\|_{L^{\infty}}^{2}
$$

provided that $0<\alpha \ll 1-\sigma$ with $0 \leq \sigma<1$.

In order to derive the $L^{2}$ estimates of the higher order for $\mathcal{G}_{2}$, one gets from $\left\langle\nabla_{v}^{k}(2.21 \mathrm{~b}), \nabla_{v}^{k} \mathbf{P}_{1} \mathcal{G}_{2}\right\rangle$ that for $k \geq 1$

$$
\begin{aligned}
\epsilon & \left\langle\nabla_{v}^{k}\left(\mathbf{P}_{1} \mathcal{G}_{2}+\mathbf{P}_{0} \mathcal{G}_{2}\right), \nabla_{v}^{k} \mathbf{P}_{1} \mathcal{G}_{2}\right\rangle-\beta^{\prime}\left\langle\nabla_{v}^{k} \nabla_{v} \cdot\left(v \mathbf{P}_{1} \mathcal{G}_{2}\right), \nabla_{v}^{k} \mathbf{P}_{1} \mathcal{G}_{2}\right\rangle \\
& -\beta^{\prime}\left\langle\nabla_{v}^{k} \nabla_{v} \cdot\left(v \mathbf{P}_{0} \mathcal{G}_{2}\right), \nabla_{v}^{k} \mathbf{P}_{1} \mathcal{G}_{2}\right\rangle-\alpha\left\langle\nabla_{v}^{k} \nabla_{v} \cdot\left(A v \mathbf{P}_{1} \mathcal{G}_{2}\right), \nabla_{v}^{k} \mathbf{P}_{1} \mathcal{G}_{2}\right\rangle \\
& -\alpha\left\langle\nabla_{v}^{k} \nabla_{v} \cdot\left(A v \mathbf{P}_{0} \mathcal{G}_{2}\right), \nabla_{v}^{k} \mathbf{P}_{1} \mathcal{G}_{2}\right\rangle+(1-\sigma)\left\langle v \nabla_{v}^{k} \mathbf{P}_{1} \mathcal{G}_{2}, \nabla_{v}^{k} \mathbf{P}_{1} \mathcal{G}_{2}\right\rangle \\
& +(1-\sigma) \sum_{1 \leq k^{\prime} \leq k} C_{k}^{k^{\prime}}\left\langle\nabla_{v}^{k^{\prime}} v \nabla_{v}^{k-k^{\prime}} \mathbf{P}_{1} \mathcal{G}_{2}, \nabla_{v}^{k} \mathbf{P}_{1} \mathcal{G}_{2}\right\rangle \\
& +(1-\sigma) \sum_{k^{\prime} \leq k} C_{k}^{k^{\prime}}\left\langle\nabla_{v}^{k^{\prime}} \nu \nabla_{v}^{k-k^{\prime}} \mathbf{P}_{0} \mathcal{G}_{2}, \nabla_{v}^{k} \mathbf{P}_{1} \mathcal{G}_{2}\right\rangle \\
& +\sigma\left\langle\nabla_{v}^{k}\left(L \mathcal{G}_{2}\right), \nabla_{v}^{k} \mathbf{P}_{1} \mathcal{G}_{2}\right\rangle-\sigma\left\langle\nabla_{v}^{k}\left[\left(1-\chi_{M}\right) \mu^{-\frac{1}{2}} \mathcal{K} \mathcal{G}_{1}\right], \nabla_{v}^{k} \mathbf{P}_{1} \mathcal{G}_{2}\right\rangle \\
=\langle & \left.\nabla_{v}^{k} \mathcal{F}_{2}, \nabla_{v}^{k} \mathbf{P}_{1} \mathcal{G}_{2}\right\rangle,
\end{aligned}
$$


from which, by using Lemma 4.3 and Cauchy-Schwarz's inequality again, we further obtain

$$
\begin{aligned}
& \epsilon\left\|\nabla_{v}^{k} \mathbf{P}_{1} \mathcal{G}_{2}\right\|^{2}+(1-\sigma)\left\|\nabla_{v}^{k} \mathbf{P}_{1} \mathcal{G}_{2}\right\|_{v}^{2}+\delta_{1} \sigma\left\|\nabla_{v}^{k} \mathbf{P}_{1} \mathcal{G}_{2}\right\|_{v}^{2}-C\left\|\mathbf{P}_{1} \mathcal{G}_{2}\right\|^{2} \\
& \leq C(\alpha+\eta)\left\|\nabla_{v}^{k} \mathbf{P}_{1} \mathcal{G}_{2}\right\|^{2}+C_{\eta} \sum_{k^{\prime}<k}\left\|\nabla_{v}^{k^{\prime}} \mathbf{P}_{1} \mathcal{G}_{2}\right\|^{2}+C \sum_{k^{\prime} \leq k}\left\|w_{l} \nabla_{v}^{k^{\prime}} \mathcal{G}_{1}\right\|_{L^{\infty}}^{2} \\
& \quad+C\left\|w_{l} \nabla_{v}^{k} \mathcal{F}_{2}\right\|_{L^{\infty}}^{2}+C\left\|\mathbf{P}_{0} \mathcal{G}_{2}\right\|^{2},
\end{aligned}
$$

where $\eta>0$ is suitably small.

As a consequence, a linear combination of (2.30) and (2.31) with $k=1, \ldots, m$ yields

$$
\begin{gathered}
\epsilon \sum_{1 \leq k \leq m}\left\|\nabla_{v}^{k} \mathbf{P}_{1} \mathcal{G}_{2}\right\|^{2}+\epsilon\left\|\mathbf{P}_{0} \mathcal{G}_{2}\right\|^{2}+\lambda \sum_{k \leq m}\left\|\nabla_{v}^{k} \mathbf{P}_{1} \mathcal{G}_{2}\right\|_{v}^{2} \\
\leq C(\epsilon) \sum_{k \leq m}\left\|w_{l} \nabla_{v}^{k} \mathcal{G}_{1}\right\|_{L^{\infty}}^{2}+C(\epsilon) \sum_{k \leq m}\left\|w_{l} \nabla_{v}^{k} \mathcal{F}_{2}\right\|_{L^{\infty}}^{2} .
\end{gathered}
$$

Finally, taking the linear combination of (2.28) and (2.32) for $0 \leq k \leq m$ and adjusting constants, we get

$$
\sum_{0 \leq k \leq m}\left\{\left\|H_{1, k}\right\|_{L^{\infty}}+\left\|H_{2, k}\right\|_{L^{\infty}}\right\} \leq C(\epsilon) \sum_{0 \leq k \leq m}\left\|w_{l} \nabla_{v}^{k}\left[\mathcal{F}_{1}, \mathcal{F}_{2}\right]\right\|_{L^{\infty}}
$$

This shows the desired estimate (2.22) and ends the proof of Lemma 2.1.

\subsection{Existence for the linear problem with fixed $\epsilon>0$}

With Lemma 2.1 in hand, we now turn to prove the existence of solutions to (2.21) with fixed $\epsilon>0$ in $L^{\infty}$ framework by the contraction mapping method.

Lemma 2.2. Let all the assumptions of Lemma 2.1 be satisfied. There is $l_{0}>0$ such that for any $l \geq l_{0}$ arbitrarily large, there are $\alpha_{0}=\alpha_{0}(l)>0$ and large $M=M(l)>0$ such that for any $0<\alpha<\alpha_{0}$, there exists a unique solution $\left[\mathcal{G}_{1}, \mathcal{G}_{2}\right] \in \overline{\mathbf{X}}_{m}$ to (2.21) with $\sigma=1$ satisfying

$$
\begin{gathered}
\sum_{0 \leq k \leq m}\left\{\left\|w_{l} \nabla_{v}^{k} \mathcal{G}_{1}\right\|_{L^{\infty}}+\left\|w_{l} \nabla_{v}^{k} \mathcal{G}_{2}\right\|_{L^{\infty}}\right\} \\
\leq C \sum_{0 \leq k \leq m}\left\{\left\|w_{l} \nabla_{v}^{k} \mathcal{F}_{1}\right\|_{L^{\infty}}+\left\|w_{l} \nabla_{v}^{k} \mathcal{F}_{2}\right\|_{L^{\infty}}\right\} .
\end{gathered}
$$


Proof. Our proof relies on the a priori estimate (2.22) established in Lemma 2.1 and the bootstrap argument, cf. [14-16].

Step 1. Existence for $\sigma=0$. If $\sigma=0$, then (2.21) becomes

$$
\begin{aligned}
& \epsilon \mathcal{G}_{1}-\beta^{\prime} \nabla_{v} \cdot\left(v \mathcal{G}_{1}\right)-\alpha \nabla_{v} \cdot\left(A v \mathcal{G}_{1}\right)+v \mathcal{G}_{1}+\frac{\beta^{\prime}}{2}|v|^{2} \sqrt{\mu} \mathcal{G}_{2} \\
& +\alpha \frac{v \cdot(A v)}{2} \sqrt{\mu} \mathcal{G}_{2}-\beta^{\prime \prime}(\mathcal{G}) \nabla_{v} \cdot(v \mu)=\mathcal{F}_{1} \\
& \epsilon \mathcal{G}_{2}-\beta^{\prime} \nabla_{v} \cdot\left(v \mathcal{G}_{2}\right)-\alpha \nabla_{v} \cdot\left(A v \mathcal{G}_{2}\right)+v \mathcal{G}_{2}=\mathcal{F}_{2} .
\end{aligned}
$$

Then, in this simple case of $\sigma=0$, since there is no trouble term involving $K$ and $\mathcal{K}$, the existence of $L^{\infty}$-solutions can be easily proved by the characteristic method and the contraction mapping theorem. That is, it follows immediately that

$$
\left\|\mathscr{L}_{0}^{-1}\left[\mathcal{G}_{1}, \mathcal{G}_{2}\right]\right\|_{\overline{\mathbf{X}}_{m}} \leq C_{\mathscr{L}}\left\|\left[\mathcal{F}_{1}, \mathcal{F}_{2}\right]\right\|_{\overline{\mathbf{X}}_{m}}
$$

Step 2. Existence for $\sigma \in\left[0, \sigma_{*}\right]$ for some $\sigma_{*}>0$. Letting $\sigma \in(0,1)$, we now consider

$$
\begin{aligned}
& \epsilon \mathcal{G}_{1}-\beta^{\prime} \nabla_{v} \cdot\left(v \mathcal{G}_{1}\right)-\alpha \nabla_{v} \cdot\left(A v \mathcal{G}_{1}\right)+v \mathcal{G}_{1}+\frac{\beta^{\prime}}{2}|v|^{2} \sqrt{\mu} \mathcal{G}_{2} \\
& \quad+\alpha \frac{v \cdot(A v)}{2} \sqrt{\mu} \mathcal{G}_{2}-\beta^{\prime \prime}(\mathcal{G}) \nabla_{v} \cdot(v \mu) \\
= & \sigma \chi_{M} \mathcal{K} \mathcal{G}_{1}+\mathcal{F}_{1} \\
& \epsilon \mathcal{G}_{2}-\beta^{\prime} \nabla_{v} \cdot\left(v \mathcal{G}_{2}\right)-\alpha \nabla_{v} \cdot\left(A v \mathcal{G}_{2}\right)+v \mathcal{G}_{2} \\
= & \sigma K \mathcal{G}_{2}+\sigma\left(1-\chi_{M}\right) \mu^{-\frac{1}{2}} \mathcal{K} \mathcal{G}_{1}+\mathcal{F}_{2},
\end{aligned}
$$

To verify the well-posedness of the above system, we further design the following approximation equations:

$$
\begin{aligned}
& \epsilon \mathcal{G}_{1}^{n+1}-\beta^{\prime} \nabla_{v} \cdot\left(v \mathcal{G}_{1}^{n+1}\right)-\alpha \nabla_{v} \cdot\left(A v \mathcal{G}_{1}^{n+1}\right)+v \mathcal{G}_{1}^{n+1} \\
& \quad+\frac{\beta^{\prime}}{2}|v|^{2} \sqrt{\mu} \mathcal{G}_{2}^{n+1}+\alpha \frac{v \cdot(A v)}{2} \sqrt{\mu} \mathcal{G}_{2}^{n+1}-\beta^{\prime \prime}\left(\mathcal{G}^{n+1}\right) \nabla_{v} \cdot(v \mu) \\
= & \sigma \chi_{M} \mathcal{K} \mathcal{G}_{1}^{n}+\mathcal{F}_{1}:=\mathcal{F}_{1}^{(1)}, \\
& \epsilon \mathcal{G}_{2}^{n+1}-\beta^{\prime} \nabla_{v} \cdot\left(v \mathcal{G}_{1}^{n+1}\right)-\alpha \nabla_{v} \cdot\left(A v \mathcal{G}_{1}^{n+1}\right)+v \mathcal{G}_{2}^{n+1} \\
= & \sigma K \mathcal{G}_{2}^{n}+\sigma\left(1-\chi_{M}\right) \mu^{-\frac{1}{2}} \mathcal{K} \mathcal{G}_{1}^{n}+\mathcal{F}_{2}:=\mathcal{F}_{2}^{(1)}
\end{aligned}
$$

with $\left[\mathcal{G}_{1}^{0}, \mathcal{G}_{2}^{0}\right]=[0,0]$. Our next goal is to prove 
(i) $\left[\mathcal{G}_{1}^{n}, \mathcal{G}_{2}^{n}\right]_{n=0}^{\infty}$ is uniformly bounded in $\overline{\mathbf{X}}_{m \text {, }}$

(ii) $\left[\mathcal{G}_{1}^{n}, \mathcal{G}_{2}^{n}\right]_{n=0}^{\infty}$ is a Cauchy sequence in $\overline{\mathbf{X}}_{m}$.

Thanks to (2.34), it follows

$$
\begin{aligned}
\left\|\left[\mathcal{G}_{1}^{n+1}, \mathcal{G}_{2}^{n+1}\right]\right\|_{\overline{\mathbf{x}}_{m}} \leq C_{\mathscr{L}}\left\|\left[\mathcal{F}_{1}^{(1)}, \mathcal{F}_{2}^{(1)}\right]\right\|_{\overline{\mathbf{x}}_{m}} \\
\leq C_{\mathscr{L}} \sigma \bar{C}_{1}\left\|\left[\mathcal{G}_{1}^{n}, \mathcal{G}_{2}^{n}\right]\right\|_{\overline{\mathbf{X}}_{m}}+\underbrace{C_{\mathscr{L}} \sum_{0 \leq k \leq m}\left\{\left\|w_{q} \nabla_{v}^{k} \mathcal{F}_{1}\right\|_{L^{\infty}}+\left\|w_{q} \nabla_{v}^{k} \mathcal{F}_{2}\right\|_{L^{\infty}}\right\}}_{\mathcal{M}_{0}},
\end{aligned}
$$

where $\bar{C}_{1}>0$ is independent of $\sigma$ and $n$. Choosing $0<\sigma_{*} \ll 1$ such that

$$
C_{\mathscr{L}} \sigma_{*} \bar{C}_{1} \leq \frac{1}{2}
$$

and moreover there exists a positive integer $N$ such that

$$
N \sigma_{*}=1 \text {. }
$$

Then we get from (2.39) that

$$
\left\|\left[\mathcal{G}_{1}^{n}, \mathcal{G}_{2}^{n}\right]\right\|_{\overline{\mathbf{X}}_{m}} \leq 2 \mathcal{M}_{0}
$$

for all $n \geq 0$. Furthermore, by (2.37), (2.38) and (2.40) and using (2.34) once more, one has

$$
\begin{aligned}
& \left\|\left[\mathcal{G}_{1}^{n+1}, \mathcal{G}_{2}^{n+1}\right]-\left[\mathcal{G}_{1}^{n}, \mathcal{G}_{2}^{n}\right]\right\|_{\overline{\mathbf{X}}_{m}} \\
\leq & C_{\mathscr{L}} \sigma \bar{C}_{1}\left\|\left[\mathcal{G}_{1}^{n}, \mathcal{G}_{2}^{n}\right]-\left[\mathcal{G}_{1}^{n-1}, \mathcal{G}_{2}^{n-1}\right]\right\|_{\overline{\mathbf{x}}_{m}} \leq \frac{1}{2}\left\|\left[\mathcal{G}_{1}^{n}, \mathcal{G}_{2}^{n}\right]-\left[\mathcal{G}_{1}^{n-1}, \mathcal{G}_{2}^{n-1}\right]\right\|_{\overline{\mathbf{X}}_{m}} .
\end{aligned}
$$

As a consequence, (2.43) and (2.42) imply that the system (2.35) and (2.36) admits a unique solution $\left[\mathcal{G}_{1}, \mathcal{G}_{2}\right] \in \overline{\mathbf{X}}_{m}$ for all $\sigma \in\left[0, \sigma_{*}\right]$. Moreover, utilizing Lemma 2.1, for such a solution, we actually have the following uniform estimate:

$$
\left\|\left[\mathcal{G}_{1}, \mathcal{G}_{2}\right]\right\|_{\overline{\mathbf{x}}_{m}} \leq C_{\mathscr{L}} \sum_{0 \leq k \leq m}\left\{\left\|w_{l} \nabla_{v}^{k} \mathcal{F}_{1}\right\|_{L^{\infty}}+\left\|w_{l} \nabla_{v}^{k} \mathcal{F}_{2}\right\|_{L^{\infty}}\right\}
$$

which is also equivalent to

$$
\left\|\mathscr{L}_{\sigma_{*}}^{-1}\left[\mathcal{F}_{1}, \mathcal{F}_{2}\right]\right\|_{\overline{\mathbf{X}}_{m}} \leq C_{\mathscr{L}}\left\|\left[\mathcal{F}_{1}, \mathcal{F}_{2}\right]\right\|_{\overline{\mathbf{X}}_{m}} .
$$


Step 3. Existence for $\sigma \in\left[0,2 \sigma_{*}\right]$ for some $\sigma_{*}>0$. By using (2.44) and performing the similar calculations as for obtaining (2.42) and (2.43), for $\sigma^{\prime} \in\left[0, \sigma_{*}\right]$, one can see that there exists a unique solution $\left[\mathcal{G}_{1}, \mathcal{G}_{2}\right] \in \overline{\mathbf{X}}_{m}$ to the lifting system

$$
\begin{aligned}
& \epsilon \mathcal{G}_{1}-\beta^{\prime} \nabla_{v} \cdot\left(v \mathcal{G}_{1}\right)-\alpha \nabla_{v} \cdot\left(A v \mathcal{G}_{1}\right)+v \mathcal{G}_{1}+\frac{\beta^{\prime}}{2}|v|^{2} \sqrt{\mu} \mathcal{G}_{2}+\alpha \frac{v \cdot(A v)}{2} \sqrt{\mu} \mathcal{G}_{2} \\
& \quad-\beta^{\prime \prime}(\mathcal{G}) \nabla_{v} \cdot(v \mu)-\sigma_{*} \chi_{M} \mathcal{K} \mathcal{G}_{1} \\
= & \sigma^{\prime} \chi_{M} \mathcal{K} \mathcal{G}_{1}+\mathcal{F}_{1} \\
& \epsilon \mathcal{G}_{2}-\beta^{\prime} \nabla_{v} \cdot\left(v \mathcal{G}_{2}\right)-\alpha \nabla_{v} \cdot\left(A v \mathcal{G}_{2}\right)+v \mathcal{G}_{2}-\sigma_{*} K \mathcal{G}_{2}-\sigma_{*}\left(1-\chi_{M}\right) \mu^{-\frac{1}{2}} \mathcal{K} \mathcal{G}_{1} \\
= & \sigma^{\prime} K \mathcal{G}_{2}+\sigma^{\prime}\left(1-\chi_{M}\right) \mu^{-\frac{1}{2}} \mathcal{K} \mathcal{G}_{1}+\mathcal{F}_{2} .
\end{aligned}
$$

In other words, we have proved the existence of $\mathscr{L}_{2 \sigma_{*}}^{-1}$ on $\overline{\mathbf{X}}_{m}$ and (2.22) holds true for $\sigma=2 \sigma_{*}$.

Step 4. Existence for $\sigma=1$. In this final step, we shall show how to extend the existence of $\mathscr{L}_{2 \sigma_{*}}^{-1}$ to the one of $\mathscr{L}_{1}^{-1}$ by the above procedure. As a matter of fact, using (2.41) and repeating Step $3(N-2)$ times, we can prove that $\mathscr{L}_{(N-1) \sigma_{*}}^{-1}$ is well-defined. With this, we then consider the following lifting system:

$$
\begin{aligned}
& \epsilon \mathcal{G}_{1}-\beta^{\prime} \nabla_{v} \cdot\left(v \mathcal{G}_{1}\right)-\alpha \nabla_{v} \cdot\left(A v \mathcal{G}_{1}\right)+v \mathcal{G}_{1}+\frac{\beta^{\prime}}{2}|v|^{2} \sqrt{\mu} \mathcal{G}_{2} \\
& +\alpha \frac{v \cdot(A v)}{2} \sqrt{\mu} \mathcal{G}_{2}-\beta^{\prime \prime}(\mathcal{G}) \nabla_{v} \cdot(v \mu)-(N-1) \sigma_{*} \chi_{M} \mathcal{K} \mathcal{G}_{1} \\
& =\sigma^{\prime} \chi_{M} \mathcal{K} \mathcal{G}_{1}+\mathcal{F}_{1} \\
& \epsilon \mathcal{G}_{2}-\beta^{\prime} \nabla_{v} \cdot\left(v \mathcal{G}_{2}\right)-\alpha \nabla_{v} \cdot\left(A v \mathcal{G}_{2}\right)+v \mathcal{G}_{2}-(N-1) \sigma_{*} K \mathcal{G}_{2} \\
& -(N-1) \sigma_{*}\left(1-\chi_{M}\right) \mu^{-\frac{1}{2}} \mathcal{K} \mathcal{G}_{1} \\
& =\sigma^{\prime} K \mathcal{G}_{2}+\sigma^{\prime}\left(1-\chi_{M}\right) \mu^{-\frac{1}{2}} \mathcal{K} \mathcal{G}_{1}+\mathcal{F}_{2} \text {, }
\end{aligned}
$$

where $\sigma^{\prime} \in\left[0, \sigma_{*}\right]$. Notice that we still have $(N-1) \sigma_{*}<1$ in the above system and as in (2.30) we may let $0<\alpha \ll 1-(N-1) \sigma_{*}$. Then as Step 2, we can further verify that $(2.45)$ and (2.46) possess a unique solution $\left[\mathcal{G}_{1}, \mathcal{G}_{2}\right] \in \overline{\mathbf{X}}_{m}$ for $\sigma^{\prime}=\sigma_{*}$. Thus $\mathscr{L}_{1}^{-1}$ is also well-defined. We emphasize that the solution we constructed here satisfies

$$
\begin{aligned}
& \epsilon \mathcal{G}_{1}-\beta^{\prime} \nabla_{v} \cdot\left(v \mathcal{G}_{1}\right)-\alpha \nabla_{v} \cdot\left(A v \mathcal{G}_{1}\right)+v \mathcal{G}_{1}+\frac{\beta^{\prime}}{2}|v|^{2} \sqrt{\mu} \mathcal{G}_{2}+\alpha \frac{v \cdot(A v)}{2} \sqrt{\mu} \mathcal{G}_{2} \\
& \quad-\beta^{\prime \prime}(\mathcal{G}) \nabla_{v} \cdot(v \mu)-\chi_{M} \mathcal{K} \mathcal{G}_{1}=\mathcal{F}_{1}, \\
& \epsilon \mathcal{G}_{2}-\beta^{\prime} \nabla_{v} \cdot\left(v \mathcal{G}_{2}\right)-\alpha \nabla_{v} \cdot\left(A v \mathcal{G}_{2}\right)+v \mathcal{G}_{2}-K \mathcal{G}_{2}-\left(1-\chi_{M}\right) \mu^{-\frac{1}{2}} \mathcal{K} \mathcal{G}_{1}=\mathcal{F}_{2},
\end{aligned}
$$


which actually implies $\left[\mathcal{G}_{1}, \mathcal{G}_{2}\right] \in \mathbf{X}_{m}$ if $\left[\mathcal{F}_{1}, \mathcal{F}_{2}\right] \in \mathbf{X}_{m}$. Therefore, by performing the similar calculation as in the next subsection, we can still show the uniform bound as (2.33). This ends the proof of Lemma 2.2.

\subsection{The remainder}

We are ready to complete the

Proof of Theorem 1.1. Since $G_{1}$ is given explicitly as (2.15), to complete the proof of Theorem 1.1, it suffices now to determine $G_{R}$ by proving the existence of the coupled system (2.16) and (2.17) under the constraint

$$
\left\langle G_{R, 1},\left[1, v_{i},|v|^{2}\right]\right\rangle+\left\langle G_{R, 2},\left[1, v_{i},|v|^{2}\right] \mu^{\frac{1}{2}}\right\rangle=0, \quad i=1,2,3
$$

To do this, let us first go back to the approximation system (2.18). By applying Lemma 2.2, for fixed $\epsilon>0$, we see that $\left[G_{R, 1}^{n+1}, G_{R, 2}^{n+1}\right]$ is well defined once $\left[G_{R, 1}^{n}, G_{R, 2}^{n}\right]$ is given and belongs to $\overline{\mathbf{X}}_{m}$ for any $m \geq 0$. Furthermore, if $\left[G_{R, 1}^{n}, G_{R, 2}^{n}\right]$ satisfies (2.47), so does $\left[G_{R, 1}^{n+1}, G_{R, 2}^{n+1}\right]$. We now verify that $\left\{G_{R, 1}^{n}, G_{R, 2}^{n}\right\}_{n=0}^{\infty}$ is a Cauchy sequence in $\mathbf{X}_{m-1}$ with $m \geq 1$, hence it is convergent and the limit denoted by $\left[G_{R, 1}^{\epsilon}, G_{R, 2}^{\epsilon}\right]$ is the unique solution of the following system:

$$
\begin{aligned}
& \quad \epsilon G_{R, 1}^{\epsilon}-\beta^{\epsilon} \nabla_{v} \cdot\left(v G_{R, 1}^{\epsilon}\right)-\alpha \nabla_{v} \cdot\left(A v G_{R, 1}^{\epsilon}\right)+v G_{R, 1}^{\epsilon} \\
& =\chi_{M} \mathcal{K} G_{R, 1}^{\epsilon}-\frac{\beta^{\epsilon}}{2}|v|^{2} \sqrt{\mu} G_{R, 2}^{\epsilon}-\frac{\alpha}{2} v \cdot(A v) \sqrt{\mu} G_{R, 2}^{\epsilon}+\frac{\beta^{\epsilon}}{\alpha} \nabla_{v} \cdot\left(v \sqrt{\mu} G_{1}\right) \\
& \quad+\beta_{1}^{\epsilon} \nabla_{v} \cdot(v \mu)+\nabla_{v} \cdot\left(A v \sqrt{\mu} G_{1}\right)+Q\left(\sqrt{\mu} G_{1}, \sqrt{\mu} G_{1}\right) \\
& \quad+\alpha\left\{Q\left(\sqrt{\mu} G_{1}, \sqrt{\mu} G_{R}^{\epsilon}\right)+Q\left(\sqrt{\mu} G_{R,}^{\epsilon} \sqrt{\mu} G_{1}\right)\right\}+\alpha^{2} Q\left(\sqrt{\mu} G_{R,}^{\epsilon} \sqrt{\mu} G_{R}^{\epsilon}\right):=\mathcal{N}_{\epsilon}, \\
& \quad \epsilon G_{R, 2}^{\epsilon}-\beta^{\epsilon} \nabla_{v} \cdot\left(v G_{R, 2}^{\epsilon}\right)-\alpha \nabla_{v} \cdot\left(A v G_{R, 2}^{\epsilon}\right)+L G_{R, 2}^{\epsilon}=\mu^{-\frac{1}{2}}\left(1-\chi_{M}\right) \mathcal{K} G_{R, 1}^{\epsilon},
\end{aligned}
$$

where

$$
\beta^{\epsilon}=\alpha \beta_{0}+\alpha^{2} \beta_{1}^{\epsilon}, \quad \beta_{1}^{\epsilon}=\frac{1}{3} \int_{\mathbb{R}^{3}} G_{1} L G_{1} d v-\frac{\alpha}{3} \int_{\mathbb{R}^{3}} \mathbf{P}_{1}\{v \cdot(A v) \sqrt{\mu}\} G_{R}^{\epsilon} d v,
$$

and

$$
\sqrt{\mu} G_{R}^{\epsilon}=G_{R, 1}^{\epsilon}+\sqrt{\mu} G_{R, 2}^{\epsilon} .
$$

The key point here is that we can prove that the convergence of the sequence $\left\{G_{R, 1}^{n}, G_{R, 2}^{n}\right\}_{n=0}^{\infty}$ is independent of $\epsilon$. To see this, we first show the following uniform bound:

$$
\left\|\left[G_{R, 1}^{n}, G_{R, 2}^{n}\right]\right\|_{\mathbf{X}_{m}} \leq 2 \mathcal{C}_{0}
$$


where $\mathcal{C}_{0}>0$ is independent of $\epsilon, n$ and $\alpha$. We give the proof by induction on $n \geq 0$. Notice that $\left[G_{R, 1}^{0}, G_{R, 2}^{0}\right]=[0,0]$, if $n=0$ the system (2.18) reads

$$
\begin{aligned}
& \epsilon G_{R, 1}^{1}-\beta^{0} \nabla_{v} \cdot\left(v G_{R, 1}^{1}\right)-\alpha \nabla_{v} \cdot\left(A v G_{R, 1}^{1}\right)+v G_{R, 1}^{1} \\
& \quad-\chi_{M} \mathcal{K} G_{R, 1}^{1}+\frac{\beta^{0}}{2}|v|^{2} \mu^{\frac{1}{2}} G_{R, 2}^{1}+\frac{\alpha}{2} v \cdot(A v) \mu^{\frac{1}{2}} G_{R, 2}^{1} \\
& \quad-\left(\beta_{1}^{1}-\frac{1}{3}\left\langle G_{1}, L G_{1}\right\rangle\right) \nabla_{v} \cdot(v \mu) \\
& =\frac{1}{3}\left\langle G_{1}, L G_{1}\right\rangle \nabla_{v} \cdot(v \mu)+\frac{\beta^{0}}{\alpha} \nabla_{v} \cdot\left(v \mu^{\frac{1}{2}} G_{1}\right) \\
& \quad+\nabla_{v} \cdot\left(A v \sqrt{\mu} G_{1}\right)+Q\left(\mu^{\frac{1}{2}} G_{1}, \mu^{\frac{1}{2}} G_{1}\right), \\
& \epsilon G_{R, 2}^{1}-\beta^{0} \nabla_{v} \cdot\left(v G_{R, 2}^{1}\right)-\alpha \nabla_{v} \cdot\left(A v G_{R, 2}^{1}\right)+L G_{R, 2}^{1} \\
& \quad-\left(1-\chi_{M}\right) \mu^{-\frac{1}{2}} \mathcal{K} G_{R, 1}^{1}=0,
\end{aligned}
$$

where $\beta^{0}$ and $\beta_{1}^{1}$ are defined as (2.19). Performing the similar calculation as for obtaining (2.28), one has

$$
\begin{aligned}
& \sum_{0 \leq k \leq m}\left\|w_{l} \nabla_{v}^{k} G_{R, 1}^{1}\right\|_{L^{\infty}} \leq C \alpha \sum_{0 \leq k \leq m}\left\|w_{l} \nabla_{v}^{k} G_{R, 2}^{1}\right\|_{L^{\infty}}+C, \\
& \sum_{0 \leq k \leq m}\left\|w_{l} \nabla_{v}^{k} G_{R, 2}^{1}\right\|_{L^{\infty}} \leq C \sum_{0 \leq k \leq m}\left\|\nabla_{v}^{k} G_{R, 2}^{1}\right\|+C \sum_{0 \leq k \leq m}\left\|w_{l} \nabla_{v}^{k} G_{R, 1}^{1}\right\|_{L^{\infty}},
\end{aligned}
$$

where the constant $C>0$ is independent of $\epsilon$.

We now turn to deduce the $H^{k}$ estimate on $G_{R, 2}^{1}$. To obtain the desired estimate which is independent of $\epsilon$, the conservation law (2.47) plays a crucial role. As a matter of fact, by the iteration scheme (2.49), it is not difficulty to check that

$$
\left\langle G_{R, 1}^{1},\left[1, v_{i},|v|^{2}\right]\right\rangle+\left\langle G_{R, 2}^{1},\left[1, v_{i},|v|^{2}\right] \mu^{\frac{1}{2}}\right\rangle=0, \quad i=1,2,3
$$

for $\epsilon>0$. We emphasize that (2.52) may not be true in the framework of (2.21) with $0<\gamma \leq 1$ and $\sigma \neq 1$.

Next, we denote for $n \geq 1$

$$
\begin{aligned}
& \overline{\mathbf{P}}_{0} G_{R, 1}^{n}=\left(a_{1}^{n}+\mathbf{b}_{1}^{n} \cdot v+c_{1}^{n}\left(|v|^{2}-3\right)\right) \mu, \\
& \mathbf{P}_{0} G_{R, 2}^{n}=\left(a_{2}^{n}+\mathbf{b}_{2}^{n} \cdot v+c_{2}^{n}\left(|v|^{2}-3\right)\right) \sqrt{\mu} .
\end{aligned}
$$

Here and in the sequel, we use the notation

$$
\mathbf{b}_{i}^{n}=\left[b_{i, 1}^{n}, b_{i, 2}^{n}, b_{i, 3}^{n}\right], \quad i=1,2 .
$$


From (2.52), one has

$$
a_{1}^{1}+a_{2}^{1}=0, \quad \mathbf{b}_{1}^{1}+\mathbf{b}_{2}^{1}=0, \quad c_{1}^{1}+c_{2}^{1}=0 .
$$

Consequently, it follows

$$
\left\|\mathbf{P}_{0} G_{R, 2}^{1}\right\| \lesssim\left|\left[a_{2}^{1}, \mathbf{b}_{2}^{1}, c_{2}^{1}\right]\right| \leq\left|\left[a_{1}^{1}, \mathbf{b}_{1}^{1}, c_{1}^{1}\right]\right| \lesssim\left\|w_{l} G_{R, 1}^{1}\right\|_{L^{\infty}}
$$

for $l>\frac{5}{2}$. On the other hand, for the microscopic component of $G_{R, 2}^{1}$, we get from the inner product $\left\langle\nabla_{v}^{k}(2.49)_{2}, \nabla_{v}^{k} \mathbf{P}_{1} G_{R, 2}^{1}\right\rangle$ with $k \geq 0$ that

$$
\begin{aligned}
\epsilon & \left\langle\nabla_{v}^{k}\left(\mathbf{P}_{1} G_{R, 2}^{1}+\mathbf{P}_{0} G_{R, 2}^{1}\right), \nabla_{v}^{k} \mathbf{P}_{1} G_{R, 2}^{1}\right\rangle-\beta^{0}\left\langle\nabla_{v}^{k} \nabla_{v} \cdot\left(v \mathbf{P}_{1} G_{R, 2}^{1}\right), \nabla_{v}^{k} \mathbf{P}_{1} G_{R, 2}^{1}\right\rangle \\
& -\beta^{0}\left\langle\nabla_{v}^{k} \nabla_{v} \cdot\left(v \mathbf{P}_{0} G_{R, 2}^{1}\right), \nabla_{v}^{k} \mathbf{P}_{1} G_{R, 2}^{1}\right\rangle-\alpha\left\langle\nabla_{v}^{k} \nabla_{v} \cdot\left(A v \mathbf{P}_{1} G_{R, 2}^{1}\right), \nabla_{v}^{k} \mathbf{P}_{1} G_{R, 2}^{1}\right\rangle \\
& -\alpha\left\langle\nabla_{v}^{k} \nabla_{v} \cdot\left(A v \mathbf{P}_{0} G_{R, 2}^{1}\right), \nabla_{v}^{k} \mathbf{P}_{1} G_{R, 2}^{1}\right\rangle+\left\langle\nabla_{v}^{k} L \mathbf{P}_{1} G_{R, 2}^{1}, \nabla_{v}^{k} \mathbf{P}_{1} G_{R, 2}^{1}\right\rangle \\
& -\left\langle\nabla_{v}^{k}\left[\left(1-\chi_{M}\right) \mu^{-\frac{1}{2}} \mathcal{K} G_{R, 1}^{1}\right], \nabla_{v}^{k} \mathbf{P}_{1} G_{R, 2}^{1}\right\rangle=0 .
\end{aligned}
$$

Using Lemma 4.3 and Cauchy-Schwarz's inequality as well as (2.54), one gets

$$
\begin{aligned}
& \left(\epsilon+\delta_{0}\right)\left\|\nabla_{v}^{k} \mathbf{P}_{1} G_{R, 2}^{1}\right\|^{2} \\
\leq & C(\epsilon+\alpha)\left\|w_{l} G_{R, 1}^{1}\right\|_{L^{\infty}}^{2}+C \sum_{k^{\prime} \leq k}\left\|w_{l} \nabla_{v}^{k^{\prime}} G_{R, 1}^{1}\right\|_{L^{\infty}}^{2}+C \mathbf{1}_{k>0}\left\|\mathbf{P}_{1} G_{R, 2}^{1}\right\|^{2} .
\end{aligned}
$$

Taking a linear combination of (2.55) with respect to $k=0,1, \ldots, m$ and applying (2.54), we arrive at

$$
\left\|\mathbf{P}_{0} G_{R, 2}^{1}\right\|^{2}+\sum_{k \leq m}\left\|\nabla_{v}^{k} \mathbf{P}_{1} G_{R, 2}^{1}\right\|^{2} \leq C \sum_{k \leq m}\left\|w_{l} \nabla_{v}^{k} G_{R, 1}^{1}\right\|_{L^{\infty}}^{2}
$$

Therefore, by plugging this into (2.51) and using (2.50), we finally obtain

$$
\sum_{0 \leq k \leq m}\left\|w_{l} \nabla_{v}^{k} G_{R, 1}^{1}\right\|_{L^{\infty}}+\sum_{0 \leq k \leq m}\left\|w_{l} \nabla_{v}^{k} G_{R, 2}^{1}\right\|_{L^{\infty}} \leq \mathcal{C}_{0}
$$

for some suitably large $\mathcal{C}_{0}>0$. This implies that (2.48) is true for $n=1$.

We now assume that (2.48) is valid for $n=N$ and then prove that (2.48) holds for $n=N+1$. In fact, applying the estimates (2.28) to the system (2.18) with $n=N$, one has

$$
\sum_{0 \leq k \leq m}\left\|w_{l} \nabla_{v}^{k} G_{R, 1}^{N+1}\right\|_{L^{\infty}}
$$




$$
\begin{aligned}
\leq & C \alpha \sum_{0 \leq k \leq m}\left\|w_{l} \nabla_{v}^{k} G_{R, 2}^{N+1}\right\|_{L^{\infty}}+C \sum_{0 \leq k \leq m}\left\|w_{l} \nabla_{v}^{k} \mathcal{S}^{N}\right\|_{L^{\infty}}, \\
& \sum_{0 \leq k \leq m}\left\|w_{l} \nabla_{v}^{k} G_{R, 2}^{N+1}\right\|_{L^{\infty}} \\
\leq & C \sum_{0 \leq k \leq m}\left\|\nabla_{v}^{k} G_{R, 2}^{N+1}\right\|+C \sum_{0 \leq k \leq m}\left\|w_{l} \nabla_{v}^{k} G_{R, 1}^{N+1}\right\|_{L^{\infty \prime}}
\end{aligned}
$$

where

$$
\begin{aligned}
\mathcal{S}^{N}= & \frac{1}{3}\left\langle G_{1}, L G_{1}\right\rangle \nabla_{v} \cdot(v \mu)+\frac{\beta^{N}}{\alpha} \nabla_{v} \cdot\left(v \mu^{\frac{1}{2}} G_{1}\right)+\nabla_{v} \cdot\left(A v \sqrt{\mu} G_{1}\right)+Q\left(\mu^{\frac{1}{2}} G_{1}, \mu^{\frac{1}{2}} G_{1}\right) \\
& +\alpha\left\{Q\left(\mu^{\frac{1}{2}} G_{R}^{N}, \mu^{\frac{1}{2}} G_{1}\right)+Q\left(\mu^{\frac{1}{2}} G_{1}, \mu^{\frac{1}{2}} G_{R}^{N}\right)\right\}+\alpha^{2} Q\left(\mu^{\frac{1}{2}} G_{R}^{N}, \mu^{\frac{1}{2}} G_{R}^{N}\right) .
\end{aligned}
$$

Recall (2.15). By employing Lemma 4.7 and the induction hypothesis, one has

$$
\sum_{0 \leq k \leq m}\left\|w_{l} \nabla_{v}^{k} \mathcal{S}^{N}\right\|_{L^{\infty}} \leq C+C \alpha \mathcal{C}_{0}+C \alpha^{2} \mathcal{C}_{0}^{2}
$$

On the other hand, since $\left[G_{R, 1}^{N}, G_{R, 2}^{N}\right] \in \mathbf{X}_{m}$, from (2.18), it also follows

$$
\left\langle G_{R, 1}^{N+1},\left[1, v_{i},|v|^{2}\right]\right\rangle+\left\langle G_{R, 2}^{N+1},\left[1, v_{i},|v|^{2}\right] \mu^{\frac{1}{2}}\right\rangle=0, \quad i=1,2,3
$$

for $\epsilon>0$. Based on this, as the estimate (2.56), one has

$$
\left\|\mathbf{P}_{0} G_{R, 2}^{N+1}\right\|^{2}+\sum_{k \leq m}\left\|\nabla_{v}^{k} \mathbf{P}_{1} G_{R, 2}^{N+1}\right\|^{2} \leq C \sum_{k \leq m}\left\|w_{l} \nabla_{v}^{k} G_{R, 1}^{N+1}\right\|_{L^{\infty}}^{2} .
$$

Substituting (2.59) and (2.60) into (2.57) and (2.58), we get

$$
\begin{aligned}
& \sum_{0 \leq k \leq m}\left\|w_{l} \nabla_{v}^{k} G_{R, 1}^{N+1}\right\|_{L^{\infty}}+\sum_{0 \leq k \leq m}\left\|w_{l} \nabla_{v}^{k} G_{R, 2}^{N+1}\right\|_{L^{\infty}} \\
\leq & C_{0}+C \alpha \mathcal{C}_{0}+C \alpha^{2} \mathcal{C}_{0}^{2} \leq 2 \mathcal{C}_{0} .
\end{aligned}
$$

Hence (2.48) is valid for all $n \geq 0$.

Having disposed of the above preliminary step, we now turn to prove that $\left.\left[G_{R, 1}^{n}, G_{R, 2}^{n}\right]\right|_{n=1} ^{\infty}$ is a Cauchy sequence in the larger function space $\mathbf{X}_{m-1}$. For this purpose, we first denote

$$
\left[\tilde{G}_{R, 1}^{n}, \tilde{G}_{R, 2}^{n}\right]=\left[G_{R, 1}^{n}-G_{R, 1}^{n-1}, G_{R, 2}^{n}-G_{R, 2}^{n-1}\right], \quad \tilde{\beta}^{n}=\beta^{n}-\beta^{n-1}, \quad n \geq 1,
$$

then by (2.18), we see that the triple $\left[\tilde{G}_{R, 1}^{n}, \tilde{G}_{R, 2}^{n}, \tilde{\beta}^{n}\right]$ satisfies

$$
\epsilon \tilde{G}_{R, 1}^{n+1}-\beta^{n} \nabla_{v} \cdot\left(v \tilde{G}_{R, 1}^{n+1}\right)-\alpha \nabla_{v} \cdot\left(A v \tilde{G}_{R, 1}^{n+1}\right)+v \tilde{G}_{R, 1}^{n+1}-\chi_{M} \mathcal{K} \tilde{G}_{R, 1}^{n+1}
$$




$$
\begin{aligned}
& \quad+\frac{\beta^{n}}{2}|v|^{2} \mu^{\frac{1}{2}} \tilde{G}_{R, 2}^{n+1}+\frac{\alpha}{2} v \cdot(A v) \mu^{\frac{1}{2}} \tilde{G}_{R, 2}^{n+1}-\tilde{\beta}_{1}^{n+1} \nabla_{v} \cdot(v \mu) \\
& =\tilde{\beta}^{n} \nabla_{v} \cdot\left(v G_{R, 1}^{n}\right)-\frac{\tilde{\beta}^{n}}{2}|v|^{2} \mu^{\frac{1}{2}} G_{R, 2}^{n}+\frac{\tilde{\beta}^{n}}{\alpha} \nabla_{v} \cdot\left(v \mu^{\frac{1}{2}} G_{1}\right) \\
& \quad+\alpha\left\{Q\left(\mu^{\frac{1}{2}} \tilde{G}_{R}^{n}, \mu^{\frac{1}{2}} G_{1}\right)+Q\left(\mu^{\frac{1}{2}} G_{1}, \mu^{\frac{1}{2}} \tilde{G}_{R}^{n}\right)\right\}+\alpha^{2} Q\left(\mu^{\frac{1}{2}} \tilde{G}_{R,}^{n}, \mu^{\frac{1}{2}} \tilde{G}_{R}^{n}\right) \\
& \quad+\alpha^{2} Q\left(\mu^{\frac{1}{2}} \tilde{G}_{R}^{n}, \mu^{\frac{1}{2}} G_{R}^{n}\right)+\alpha^{2} Q\left(\mu^{\frac{1}{2}} G_{R}^{n}, \mu^{\frac{1}{2}} \bar{G}_{R}^{n}\right), \\
& \epsilon \tilde{G}_{R, 2}^{n+1}-\beta^{n} \nabla_{v} \cdot\left(v \tilde{G}_{R, 2}^{n+1}\right)-\alpha \nabla_{v} \cdot\left(A v \tilde{G}_{R, 2}^{n+1}\right)+L \tilde{G}_{R, 2}^{n+1}-\left(1-\chi_{M}\right) \mu^{-\frac{1}{2}} \mathcal{K} \tilde{G}_{R, 1}^{n+1} \\
& =\tilde{\beta}^{n} \nabla_{v} \cdot\left(v G_{R, 2}^{n}\right) .
\end{aligned}
$$

Note that from (2.19) one actually has $\tilde{\beta}^{n}=\alpha^{2} \tilde{\beta}_{1}^{n}=\alpha^{2}\left(\beta_{1}^{n}-\beta_{1}^{n-1}\right)$. Since both $\left[G_{R, 1}^{n}, G_{R, 2}^{n}\right]$ and $\left[G_{R, 1}^{n+1}, G_{R, 2}^{n+1}\right]$ satisfy $(2.47)$, so does their difference $\left[\tilde{G}_{R, 1}^{n+1}, \tilde{G}_{R, 2}^{n+1}\right]$. With this, we can proceed analogously to the deduction of (2.61) to obtain that

$$
\begin{gathered}
\sum_{k \leq m-1}\left\{\left\|w_{l} \nabla_{v}^{k} \tilde{G}_{R, 1}^{n+1}\right\|_{L^{\infty}}+\left\|w_{l} \nabla_{v}^{k} \tilde{G}_{R, 2}^{n+1}\right\|_{L^{\infty}}\right\} \\
\leq C \alpha\left|\tilde{\beta}_{1}^{n}\right|+C \alpha \sum_{k \leq m-1}\left\{\left\|w_{l} \tilde{G}_{R, 1}^{n}\right\|_{L^{\infty}}+\left\|w_{l} \tilde{G}_{R, 2}^{n}\right\|_{L^{\infty}}\right\} \\
\quad+C \alpha \sum_{k \leq m-1}\left\{\left\|w_{l} \tilde{G}_{R, 1}^{n}\right\|_{L^{\infty}}+\left\|w_{l} \tilde{G}_{R, 2}^{n}\right\|_{L^{\infty}}\right\}^{2},
\end{gathered}
$$

which is equivalent to

$$
\left\|\left[\tilde{G}_{R, 1}^{n+1}, \tilde{G}_{R, 2}^{n+1}\right]\right\|_{\mathbf{X}_{m-1}} \leq C \alpha\left\|\left[\tilde{G}_{R, 1}^{n}, \tilde{G}_{R, 2}^{n}\right]\right\|_{\mathbf{X}_{m-1}} .
$$

Therefore $\left[G_{R, 1}^{n}, G_{R, 2}^{n}\right]$ converges strongly to some function pair $\left[G_{R, 1}^{\epsilon}, G_{R, 2}^{\epsilon}\right] \in \mathbf{X}_{m-1}$. Moreover, from (2.48), it also follows

$$
\left\|\left[G_{R, 1}^{\epsilon}, G_{R, 2}^{\epsilon}\right]\right\|_{\mathbf{X}_{m}} \leq 2 \mathcal{C}_{0}
$$

We shall have established the theorem if we prove that $\left[G_{R, 1}^{\epsilon}, G_{R, 2}^{\epsilon}\right] \rightarrow\left[G_{R, 1}, G_{R, 2}\right]$ as $\epsilon \rightarrow 0^{+}$. For this, we choose a positive sequence $\left\{\epsilon_{n}\right\}_{n=1}^{\infty}$ such that $\left|\epsilon_{n+1}-\epsilon_{n}\right| \leq 2^{-n}$, then $\epsilon_{n} \rightarrow 0^{+}$as $n \rightarrow+\infty$. We consider the following approximation equations:

$$
\begin{aligned}
& \epsilon_{n} G_{R, 1}^{\epsilon_{n}}-\beta^{\epsilon_{n}} \nabla_{v} \cdot\left(v G_{R, 1}^{\epsilon_{n}}\right)-\alpha \nabla_{v} \cdot\left(A v G_{R, 1}^{\epsilon_{n}}\right)+v G_{R, 1}^{\epsilon_{n}} \\
& =\chi_{M} \mathcal{K} G_{R, 1}^{\epsilon_{n}}-\frac{\beta^{\epsilon_{n}}}{2}|v|^{2} \sqrt{\mu} G_{R, 2}^{\epsilon_{n}}-\frac{\alpha}{2} v \cdot(A v) \sqrt{\mu} G_{R, 2}^{\epsilon_{n}}+\frac{\beta^{\epsilon_{n}}}{\alpha} \nabla_{v} \cdot\left(v \sqrt{\mu} G_{1}\right) \\
& \quad+\beta_{1}^{\epsilon_{n}} \nabla_{v} \cdot(v \mu)+\nabla_{v} \cdot\left(A v \sqrt{\mu} G_{1}\right)+Q\left(\sqrt{\mu} G_{1}, \sqrt{\mu} G_{1}\right)
\end{aligned}
$$




$$
\begin{array}{r}
+\alpha\left\{Q\left(\sqrt{\mu} G_{1}, \sqrt{\mu} G_{R}^{\epsilon}\right)+Q\left(\sqrt{\mu} G_{R}^{\epsilon_{n}}, \sqrt{\mu} G_{1}\right)\right\}+\alpha^{2} Q\left(\sqrt{\mu} G_{R}^{\epsilon_{n}}, \sqrt{\mu} G_{R}^{\epsilon_{n}}\right), \\
\epsilon_{n} G_{R, 2}^{\epsilon_{n}}-\beta^{\epsilon_{n}} \nabla_{v} \cdot\left(v G_{R, 2}^{\epsilon_{n}}\right)-\alpha \nabla_{v} \cdot\left(A v G_{R, 2}^{\epsilon_{n}}\right)+L G_{R, 2}^{\epsilon_{n}}=\mu^{-\frac{1}{2}}\left(1-\chi_{M}\right) \mathcal{K} G_{R, 1}^{\epsilon_{n}} .
\end{array}
$$

Since each pair $\left[G_{R, 1}^{\epsilon_{n}}, G_{R, 2}^{\epsilon_{n}}\right]$ is well-defined and satisfies (2.63), we have as the estimate (2.62) that

$$
\left\|\left[G_{R, 1}^{\epsilon_{n}}-G_{R, 1}^{\epsilon_{n-1}}, G_{R, 2}^{\epsilon_{n}}-G_{R, 2}^{\epsilon_{n-1}}\right]\right\|_{\mathbf{X}_{m-1}} \leq C\left|\epsilon_{n}-\epsilon_{n-1}\right|, \quad n \geq 1 .
$$

Thus $\left[G_{R, 1}^{\epsilon_{n}}, G_{R, 2}^{\epsilon_{n}}\right] \rightarrow\left[G_{R, 1}, G_{R, 2}\right]$ as $\epsilon_{n} \rightarrow 0^{+}$. Moreover it holds that $\left[G_{R, 1}, G_{R, 2}\right] \in \mathbf{X}_{m}$ satisfies the same estimate as (2.63). This proves (1.12). The non-negativity of the steady solution $G_{s t}=\mu+\alpha \sqrt{\mu}\left(G_{1}+\alpha G_{R}\right)$ constructed here is a direct subsequence of the dynamical stability of $G_{s t}(v)$ verified in Theorem 1.2. This ends the proof of Theorem 1.1.

\section{Unsteady problem}

In this section, we turn to the time-dependent case. Our goal is to prove that the large time behavior of the Cauchy problem (1.13) and (1.14) can be governed by the steady problem (1.1) which has been solved in Section 2 . The proof is based on the local-in-time existence and the a priori estimate as well as the continuum argument.

The local-in-time existence of the Cauchy problem (1.13) and (1.14) will be established by an iteration method and Duhamel's principle. Set $G=G_{s t}+\sqrt{\mu} f$, then we see that $f$ satisfies

$$
\begin{aligned}
& \partial_{t} f+v \cdot \nabla_{x} f-\beta \mu^{-\frac{1}{2}} \nabla_{v} \cdot(v \sqrt{\mu} f)-\alpha \mu^{-\frac{1}{2}} \nabla_{v} \cdot(A v \sqrt{\mu} f)+L f \\
= & \Gamma(f, f)+\alpha\left\{\Gamma\left(G_{1}+\alpha G_{R}, f\right)+\Gamma\left(f, G_{1}+\alpha G_{R}\right)\right\}, \quad t>0, \quad x \in \mathbb{T}^{3}, \quad v \in \mathbb{R}^{3}
\end{aligned}
$$

with

$$
\sqrt{\mu} f(0, x, v) \stackrel{\text { def }}{=} f_{0}(x, v)=G_{0}(x, v)-G_{s t}(v), \quad x \in \mathbb{T}^{3}, \quad v \in \mathbb{R}^{3} .
$$

As it is pointed out in Section 2, to eliminate the severe velocity growth in the left hand side of (3.1), it is necessary to use the following Caflisch's decomposition:

$$
\sqrt{\mu} f=f_{1}+\sqrt{\mu} f_{2}
$$

where $f_{1}$ and $f_{2}$ satisfy

$$
\partial_{t} f_{1}+v \cdot \nabla_{x} f_{1}-\beta \nabla_{v} \cdot\left(v f_{1}\right)-\alpha \nabla_{v} \cdot\left(A v f_{1}\right)+v f_{1}
$$




$$
\begin{aligned}
& =\chi_{M} \mathcal{K} f_{1}-\frac{\beta}{2}|v|^{2} \sqrt{\mu} f_{2}-\frac{\alpha}{2} v \cdot(A v) \sqrt{\mu} f_{2}+\alpha A v \cdot\left(\nabla_{v} \sqrt{\mu}\right)\left(|v|^{2}-3\right) \sqrt{\mu} c_{f_{2}} \\
& \quad+Q\left(f_{1}, f_{1}\right)+Q\left(f_{1}, \sqrt{\mu} f_{2}\right)+Q\left(\sqrt{\mu} f_{2}, f_{1}\right) \\
& \quad+\alpha\left\{Q\left(\sqrt{\mu}\left(G_{1}+\alpha G_{R}\right), \sqrt{\mu} f\right)+Q\left(\sqrt{\mu} f, \sqrt{\mu}\left(G_{1}+\alpha G_{R}\right)\right)\right\}, \\
& \quad t>0, \quad x \in \mathbb{T}^{3}, \quad v \in \mathbb{R}^{3}, \\
& f_{1}(0, x, v)=f_{0}(x, v)=G_{0}(x, v)-G_{s t}(v), \quad x \in \mathbb{T}^{3}, \quad v \in \mathbb{R}^{3}, \\
& \partial_{t} f_{2}+v \cdot \nabla_{x} f_{2}-\beta \nabla_{v} \cdot\left(v f_{2}\right)-\alpha \nabla_{v} \cdot\left(A v f_{2}\right) \\
& \quad+\alpha A v \cdot\left(\nabla_{v} \sqrt{\mu}\right)\left(|v|^{2}-3\right) c_{f_{2}}+L f_{2} \\
& =\left(1-\chi_{M}\right) \mu^{-\frac{1}{2}} \mathcal{K} f_{1}+\Gamma\left(f_{2}, f_{2}\right), \quad t>0, \quad x \in \mathbb{T}^{3}, \quad v \in \mathbb{R}^{3},
\end{aligned}
$$

and

$$
f_{2}(0, x, v)=0, \quad x \in \mathbb{T}^{3}, \quad v \in \mathbb{R}^{3},
$$

respectively. Here, $c_{f_{2}}$ is defined as

$$
\mathbf{P}_{0} f_{2}=\left\{a_{f_{2}}(t, x)+\mathbf{b}_{f_{2}}(t, x) \cdot v+c_{f_{2}}(t, x)\left(|v|^{2}-3\right)\right\} \sqrt{\mu} .
$$

To determine $f$, we instead turn to solve $f_{1}$ and $f_{2}$ through the above system.

We shall look for solutions of (3.3)-(3.6) in the following function space:

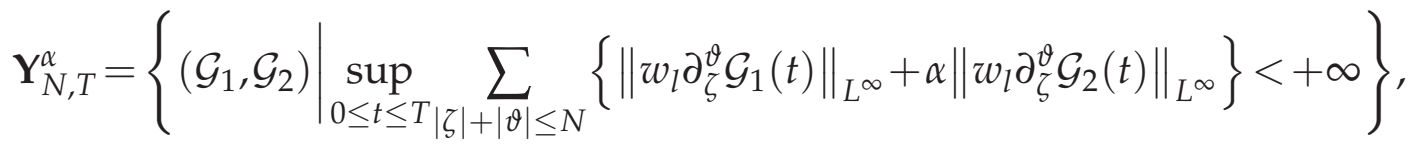

associated with the norm

$$
\left\|\left[\mathcal{G}_{1}, \mathcal{G}_{2}\right]\right\|_{\mathbf{Y}_{N, T}^{\alpha}}=\sup _{0 \leq t \leq T|\zeta|+|\vartheta| \leq N} \sum_{0}\left\{\left\|w_{l} \partial_{\zeta}^{\vartheta} \mathcal{G}_{1}(t)\right\|_{L^{\infty}}+\alpha\left\|w_{l} \partial_{\zeta}^{\vartheta} \mathcal{G}_{2}(t)\right\|_{L^{\infty}}\right\} .
$$

We then have the following result on local-in-time existence. For brevity, we omit its proof, cf. [14].

Theorem 3.1 (Local existence). Under the conditions stated in Theorem 1.2, there exits $T_{*}>0$ which may depend on $\alpha$ such that the coupling problem (3.3)-(3.6) admits a unique local in time solution $\left[f_{1}(t, x, v), f_{2}(t, x, v)\right]$ satisfying

$$
\left\|\left[f_{1}, f_{2}\right]\right\|_{\mathbf{Y}_{N, T_{*}}^{\alpha}} \leq C_{0} \alpha^{2}
$$

for a constant $C_{0}>0$ independent of $\alpha$. 
In what follows we focus on deducing the a priori $W^{N, \infty}$ estimates on the solution constructed in Theorem 3.1. Namely, we assume that $\left[f_{1}, f_{2}\right]$ is a classical solution to the initial value problem (3.3)-(3.6). The purpose is to prove

$$
\begin{aligned}
& \sup _{0 \leq s \leq t|\zeta|+|\vartheta| \leq N} \sum e^{\lambda_{0} s}\left\|w_{l} \partial_{\zeta}^{\vartheta} f_{1}(s)\right\|_{L^{\infty}} \\
& \quad+\alpha \sup _{0 \leq s \leq t|\zeta|+|\vartheta| \leq N} \sum^{\lambda_{0} s}\left\|w_{l} \partial_{\zeta}^{\vartheta} f_{2}(s)\right\|_{L^{\infty}} \\
& \leq C \sum_{|\zeta|+|\vartheta| \leq N}\left\|w_{l} \partial_{\zeta}^{\vartheta} f_{0}\right\|_{L^{\infty}}
\end{aligned}
$$

for any $t \geq 0$ and some constant $C>0$, under the a priori assumption that

$$
\begin{aligned}
& \sup _{0 \leq s \leq t|\zeta|+|\vartheta| \leq N} \sum_{0 \leq s \leq t|\zeta|+|\vartheta| \leq N} e^{\lambda_{0} s}\left\|w_{l} \partial_{\zeta}^{\vartheta} f_{1}(s)\right\|_{L^{\infty}} \\
& \quad+\alpha \sup _{0 \leq \lambda^{\prime} s} \sum_{\lambda_{l} \partial_{\zeta}^{\vartheta} f_{2}(s) \|_{L^{\infty}} \leq \alpha^{2},}
\end{aligned}
$$

where $\lambda_{0}>0$ is independent of $\alpha$ to be determined later. Note that the initial condition (1.16) is the consequence of (3.8). The a priori estimate together with the local existence established in Theorem 3.1 and the continuum argument enables us to construct the global existence for the Cauchy problem (3.1) and (3.2). Thus we are ready to complete the

Proof of Theorem 1.2. We first verify that (3.8) holds true under the a priori assumption (3.7). The proof is divided into two steps.

Step 1. $W^{k, \infty}$ estimates. Denoting

$$
\left[g_{1}, g_{2}\right](t)=e^{\lambda_{0} t}\left[f_{1}, f_{2}\right](t),
$$

and defining

$$
\mathbf{P}_{0 g_{2}}=\left\{a_{2}(t, x)+\mathbf{b}_{2}(t, x) \cdot v+c_{2}(t, x)\left(|v|^{2}-3\right)\right\} \sqrt{\mu},
$$

one has by (3.3)-(3.6) that

$$
\begin{aligned}
\partial_{t} & {\left[w_{l} \partial_{\zeta}^{\vartheta} g_{1}\right]+v \cdot \nabla_{x}\left[w_{l} \partial_{\zeta}^{\vartheta} g_{1}\right]-\beta v \cdot \nabla_{v}\left[w_{l} \partial_{\zeta}^{\vartheta} g_{1}\right]+2 l \beta \frac{|v|^{2}}{1+|v|^{2}} w_{l} \partial_{\zeta}^{\vartheta} g_{1}-3 \beta w_{l} \partial_{\zeta}^{\vartheta} g_{1} } \\
& -\alpha A v \cdot \nabla_{v}\left[w_{l} \partial_{\zeta}^{\vartheta} g_{1}\right]+2 l \alpha \frac{v \cdot A v}{1+|v|^{2}} w_{l} \partial_{\zeta}^{\vartheta} g_{1}-\alpha \operatorname{tr} A w_{l} \partial_{\zeta}^{\vartheta} g_{1}-\lambda_{0} w_{l} \partial_{\zeta}^{\vartheta} g_{1}+v w_{l} \partial_{\zeta}^{\vartheta} g_{1}
\end{aligned}
$$




$$
\begin{aligned}
& =-\mathbf{1}_{|\zeta|>0} \sum_{\left|\zeta^{\prime}\right|=1} C_{\zeta}^{\zeta^{\prime}} w_{l} \partial_{\zeta^{\prime}} v \cdot \nabla_{x} \partial_{\zeta-\zeta^{\prime}}^{\vartheta} g_{1}+\beta \mathbf{1}_{|\zeta|>0} \sum_{\left|\zeta^{\prime}\right|=1} C_{\zeta}^{\zeta^{\prime}} w_{l} \partial_{\zeta^{\prime}} v \cdot \nabla_{v} \partial_{\zeta-\zeta^{\prime}}^{\vartheta} g_{1} \\
& +\alpha \mathbf{1}_{|\zeta|>0} \sum_{\left|\zeta^{\prime}\right|=1} C_{\zeta}^{\zeta^{\prime}} w_{l} \partial_{\zeta^{\prime}}(A v) \cdot \nabla_{v} \partial_{\zeta-\zeta^{\prime}}^{\vartheta} g_{1} \\
& -\mathbf{1}_{|\zeta|>0} w_{l} \sum_{0<\zeta^{\prime} \leq \zeta} C_{\zeta}^{\zeta^{\prime}} w_{l} \partial_{\zeta-\zeta^{\prime}} \nu \partial_{\zeta-\zeta^{\prime}}^{\vartheta} g_{1}+w_{l} \partial_{\zeta}^{\vartheta}\left(\chi_{M} \mathcal{K} g_{1,0}\right)-\frac{\beta}{2} w_{l} \partial_{\zeta}^{\vartheta}\left(|v|^{2} \sqrt{\mu} g_{2}\right) \\
& -\frac{\alpha}{2} w_{l} \partial_{\zeta}^{\vartheta}\left(v \cdot(A v) \sqrt{\mu} g_{2}\right)+\alpha w_{l} \partial_{\zeta}^{\vartheta}\left(A v \cdot\left(\nabla_{v} \sqrt{\mu}\right)\left(|v|^{2}-3\right) \sqrt{\mu} c_{2}\right) \\
& +e^{\lambda_{0} t} w_{l} \partial_{\zeta}^{\vartheta}\left\{Q\left(f_{1}, f_{1}\right)+Q\left(f_{1}, \sqrt{\mu} f_{2}\right)+Q\left(\sqrt{\mu} f_{2}, f_{1}\right)\right\} \\
& +\alpha w_{l} e^{\lambda_{0} t} \partial_{\zeta}^{\vartheta}\left\{Q\left(\sqrt{\mu}\left(G_{1}+\alpha G_{R}\right), \sqrt{\mu} f\right)+Q\left(\sqrt{\mu} f, \sqrt{\mu}\left(G_{1}+\alpha G_{R}\right)\right)\right\}, \\
& \partial_{\zeta}^{\vartheta} g_{1}(0, x, v)=\partial_{\zeta}^{\vartheta} f_{0}(x, v) \\
& \partial_{t}\left[w_{l} \partial_{\zeta}^{\vartheta} g_{2}\right]+v \cdot \nabla_{x}\left[w_{l} \partial_{\zeta}^{\vartheta} g_{2}\right]-\beta v \cdot \nabla_{v}\left[w_{l} \partial_{\zeta}^{\vartheta} g_{2}\right]-2 l \beta \frac{|v|^{2}}{1+|v|^{2}} w_{l} \partial_{\zeta}^{\vartheta} g_{2}-3 \beta w_{l} \partial_{\zeta}^{\vartheta} g_{2} \\
& -\alpha A v \cdot \nabla_{v}\left[w_{l} \partial_{\zeta}^{\vartheta} g_{2}\right]+2 l \alpha \frac{v \cdot A v}{1+|v|^{2}} w_{l} \partial_{\zeta}^{\vartheta} g_{2}-\alpha \operatorname{tr} A w_{l} \partial_{\zeta}^{\vartheta} g_{2}-\lambda_{0} w_{l} \partial_{\zeta}^{\vartheta} g_{2}+v w_{l} \partial_{\zeta}^{\vartheta} g_{2} \\
& =-\mathbf{1}_{|\zeta|>0} \sum_{\left|\zeta^{\prime}\right|=1} C_{\zeta}^{\zeta^{\prime}} w_{l} \partial_{\zeta^{\prime}} v \cdot \nabla_{x} \partial_{\zeta-\zeta^{\prime}}^{\vartheta} g_{2}+\beta \mathbf{1}_{|\zeta|>0} \sum_{\left|\zeta^{\prime}\right|=1} C_{\zeta}^{\zeta^{\prime}} w_{l} \partial_{\zeta^{\prime}} v \cdot \nabla_{v} \partial_{\zeta-\zeta^{\prime}}^{\vartheta} g_{2} \\
& +\alpha \mathbf{1}_{\zeta>0} \sum_{\left|\zeta^{\prime}\right|=1} C_{\zeta}^{\zeta^{\prime}} w_{l} \partial_{\zeta^{\prime}}(A v) \cdot \nabla_{v} \partial_{\zeta-\zeta^{\prime}}^{\vartheta} g_{2}-\alpha w_{l} \partial_{\zeta}^{\vartheta}\left(A v \cdot\left(\nabla_{v} \sqrt{\mu}\right)\left(|v|^{2}-3\right) c_{2}\right) \\
& -\mathbf{1}_{|\zeta|>0} \sum_{0<\zeta^{\prime} \leq \zeta} C_{\zeta}^{\zeta^{\prime}} w_{l} \partial_{\zeta^{\prime}} \nu \partial_{\zeta}^{\vartheta}-\zeta^{\prime} g_{2}+w_{l} \partial_{\zeta}^{\vartheta}\left(K g_{2,0}\right)+w_{l} \partial_{\zeta}^{\vartheta}\left(\left(1-\chi_{M}\right) \mu^{-\frac{1}{2}} \mathcal{K} g_{1}\right) \\
& +e^{\lambda_{0} t} w_{l} \partial_{\zeta}^{\vartheta} \Gamma\left(f_{2}, f_{2}\right)
\end{aligned}
$$

and

$$
\partial_{\zeta}^{\vartheta} g_{2}(0, x, v)=0
$$

As in (2.25), we recall that the characteristic line of the above system can be determined by

$$
\begin{aligned}
& \frac{d X}{d s}=V(s ; t, x, v), \\
& \frac{d V}{d s}=-\beta V(s ; t, x, v)-\alpha A V(s ; t, x, v), \\
& X(t ; t, x, v)=x, \quad V(t ; t, x, v)=v,
\end{aligned}
$$


which gives

$$
\begin{aligned}
& V(s)=V(s ; t, x, v)=e^{-(s-t)(\beta I+\alpha A)} v \\
& X(s)=X(s ; t, x, v)=x-(\beta I+\alpha A)^{-1}\left[e^{-(s-t)(\beta I+\alpha A)}-I\right] v .
\end{aligned}
$$

Along the characteristic line (3.11), we write the solution of (3.9) and (3.10) as the following mild form:

with

$$
w_{l} \partial_{\zeta}^{\vartheta} g_{1}(t, x, v)=\sum_{i=1}^{9} \mathcal{H}_{i}
$$

$$
\begin{aligned}
& \mathcal{H}_{1}= e^{-\int_{0}^{t} \mathcal{A}^{\lambda}(s) d s} w_{l} \partial_{\zeta}^{\vartheta} f_{0}(X(0), V(0)), \\
& \mathcal{H}_{2}=-\mathbf{1}_{|\zeta|>0} \sum_{\left|\zeta^{\prime}\right|=1} C_{\zeta}^{\zeta^{\prime}} \int_{0}^{t} e^{-\int_{s}^{t} \mathcal{A}^{\lambda}(\tau) d \tau}\left\{w_{l} \partial_{\zeta^{\prime}} v \cdot \nabla_{x} \partial_{\zeta}^{\vartheta}-\zeta^{\prime} g_{1}\right\}(s, X(s), V(s)) d s, \\
& \mathcal{H}_{3}= \beta \mathbf{1}_{|\zeta|>0} \sum_{\left|\zeta^{\prime}\right|=1} C_{\zeta}^{\zeta^{\prime}} \int_{0}^{t} e^{-\int_{s}^{t} \mathcal{A}^{\lambda}(\tau) d \tau}\left\{w_{l} \partial_{\zeta^{\prime}} v \cdot \nabla_{v} \partial_{\zeta}^{\vartheta}-\zeta^{\prime} g_{1}\right\}(s, X(s), V(s)) d s, \\
& \mathcal{H}_{4}= \alpha \mathbf{1}_{|\zeta|>0} \sum_{\left|\zeta^{\prime}\right|=1} C_{\zeta}^{\zeta^{\prime}} \int_{0}^{t} e^{-\int_{s}^{t} \mathcal{A}^{\lambda}(\tau) d \tau}\left\{w_{l} \partial_{\zeta^{\prime}}(A v) \cdot \nabla_{v} \partial_{\zeta-\zeta^{\prime}}^{\vartheta} g_{1}\right\}(s, X(s), V(s)) d s, \\
& \mathcal{H}_{5}=-\mathbf{1}_{|\zeta|>0} \sum_{0<\zeta^{\prime} \leq \zeta} C_{\zeta}^{\zeta^{\prime}} \int_{0}^{t} e^{-\int_{s}^{t} \mathcal{A}^{\lambda}(\tau) d \tau}\left\{w_{l} \partial_{\zeta^{\prime}} v \partial_{\zeta}^{\vartheta}-\zeta^{\prime} g_{1}\right\}(s, X(s), V(s)) d s, \\
& \mathcal{H}_{6}= \int_{0}^{t} e^{-\int_{s}^{t} \mathcal{A}^{\lambda}(\tau) d \tau}\left\{w_{l} \partial_{\zeta}^{\vartheta}\left(\chi_{M} \mathcal{K} g_{1}\right)\right\}(s, X(s), V(s)) d s, \\
& \mathcal{H}_{7}=- \int_{0}^{t} e^{\int_{s}^{t} \mathcal{A}^{\lambda}(\tau) d \tau}\left\{\frac{\beta}{2} w_{l} \partial_{\zeta}^{\vartheta}\left(|v|^{2} \sqrt{\mu} g_{2}\right)+\frac{\alpha}{2} w_{l} \partial_{\zeta}^{\vartheta}\left(v \cdot(A v) \sqrt{\mu} g_{2}\right)\right. \\
&\left.-\alpha w_{l} \partial_{\zeta}^{\vartheta}\left(A v \cdot\left(\nabla_{v} \sqrt{\mu}\right)\left(|v|^{2}-3\right) \sqrt{\mu} c_{2}\right)\right\}(s, X(s), V(s)) d s, \\
& \mathcal{H}_{8}= \int_{0}^{t} e^{-\int_{s}^{t} \mathcal{A}^{\lambda}(\tau) d \tau} e^{\lambda_{0} s}\left\{w_{l} \partial_{\zeta}^{\vartheta}\left\{Q\left(f_{1}, f_{1}\right)+Q\left(f_{1}, \sqrt{\mu} f_{2}\right)+Q\left(\sqrt{\mu} f_{2}, f_{1}\right)\right\}\right\} \\
& \times(s, X(s), V(s)) d s, \\
& \mathcal{H}_{9}=\alpha \int_{0}^{t} e^{-\int_{s}^{t} \mathcal{A}^{\lambda}(\tau) d \tau} e^{\lambda_{0} s}\left\{w _ { l } \partial _ { \zeta } ^ { \vartheta } \left\{Q\left(\sqrt{\mu}\left(G_{1}+\alpha G_{R}\right), \sqrt{\mu} f\right)\right.\right. \\
&\left.\left.+Q\left(\sqrt{\mu} f, \sqrt{\mu}\left(G_{1}+\alpha G_{R}\right)\right)\right\}\right\}(s, X(s), V(s)) d s,
\end{aligned}
$$

and

$$
w_{l} \partial_{\zeta}^{\vartheta} g_{2}(t, x, v)=\sum_{i=10}^{16} \mathcal{H}_{i}
$$


with

$$
\begin{aligned}
& \mathcal{H}_{10}=-\mathbf{1}_{|\zeta|>0} \sum_{\left|\zeta^{\prime}\right|=1} C_{\zeta}^{\zeta^{\prime}} \int_{0}^{t} e^{-\int_{s}^{t} \mathcal{A}^{\lambda}(\tau) d \tau}\left\{w_{l} \partial_{\zeta^{\prime}} v \cdot \nabla_{x} \partial_{\zeta}^{\vartheta}-\zeta^{\prime} g_{2}\right\}(s, X(s), V(s)) d s, \\
& \mathcal{H}_{11}=\mathbf{1}_{|\zeta|>0} \sum_{\left|\zeta^{\prime}\right|=1} C_{\zeta}^{\zeta^{\prime}} \int_{0}^{t} e^{-\int_{s}^{t} \mathcal{A}^{\lambda}(\tau) d \tau}\left\{\beta w_{l} \partial_{\zeta^{\prime}} v \cdot \nabla_{v} \partial_{\zeta-\zeta^{\prime}}^{\vartheta} g_{2}+\alpha w_{l} \partial_{\zeta^{\prime}}(A v) \cdot \nabla_{v} \partial_{\zeta-\zeta^{\prime}}^{\vartheta} g_{2}\right. \\
& \left.\quad-\alpha w_{l} \partial_{\zeta}^{\vartheta}\left(A v \cdot\left(\nabla_{v} \sqrt{\mu}\right)\left(|v|^{2}-3\right) c_{2}\right)\right\}(s, X(s), V(s)) d s, \\
& \mathcal{H}_{12}=-\mathbf{1}_{|\zeta|>0} \sum_{0<\zeta_{1} \leq \zeta} C_{\zeta}^{\zeta^{\prime}} \int_{0}^{t} e^{-\int_{s}^{t} \mathcal{A}^{\lambda}(\tau) d \tau}\left\{w_{l} \partial_{\zeta^{\prime}} v \partial_{\zeta-\zeta_{1}}^{\vartheta} g_{2}\right\}(s, X(s), V(s)) d s, \\
& \mathcal{H}_{13}=\int_{0}^{t} e^{-\int_{s}^{t} \mathcal{A}^{\lambda}(\tau) d \tau}\left\{w_{l} K \partial_{\zeta}^{\vartheta} g_{2}\right\}(s, X(s), V(s)) d s, \\
& \mathcal{H}_{14}=\mathbf{1}_{|\zeta|>0} \sum_{0<\zeta^{\prime} \leq \zeta} C_{\zeta}^{\zeta^{\prime}} \int_{0}^{t} e^{-\int_{s}^{t} \mathcal{A}^{\lambda}(\tau) d \tau}\left\{w_{l}\left(\partial_{\zeta^{\prime}} K\right)\left(\partial_{\zeta-\zeta^{\prime}}^{\vartheta} g_{2}\right)\right\}(s, X(s), V(s)) d s, \\
& \mathcal{H}_{15}=\int_{0}^{t} e^{-\int_{s}^{t} \mathcal{A}^{\lambda}(\tau) d \tau}\left\{w_{l} \partial_{\zeta}^{\vartheta}\left(\left(1-\chi_{M}\right) \mu^{-\frac{1}{2}} \mathcal{K} g_{1}\right)\right\}(s, X(s), V(s)) d s, \\
& \mathcal{H}_{16}=\int_{0}^{t} e^{-\int_{s}^{t} \mathcal{A}^{\lambda}(\tau) d \tau} e^{\lambda_{0} s}\left\{w_{l} \partial_{\zeta}^{\vartheta} \Gamma\left(f_{2}, f_{2}\right)\right\}(s, X(s), V(s)) d s .
\end{aligned}
$$

Here, as before we have denoted

$$
\begin{aligned}
\mathcal{A}^{\lambda}(\tau, V(\tau)) & =v(V(\tau))-3 \beta+2 l \beta \frac{|V(\tau)|^{2}}{1+|V(\tau)|^{2}}+2 l \alpha \frac{V(\tau) \cdot(A V(\tau))}{1+|V(\tau)|^{2}}-\alpha \operatorname{tr} A-\lambda_{0} \\
& =v(V(\tau))-3 \beta_{1}+2 l \beta \frac{|V(\tau)|^{2}}{1+|V(\tau)|^{2}}+2 l \alpha \frac{V(\tau) \cdot(A V(\tau))}{1+|V(\tau)|^{2}}-\lambda_{0},
\end{aligned}
$$

and moreover, as long as $l \alpha>0,|l \beta|$ and $\lambda_{0}$ are suitably small, one sees that $\mathcal{A}^{\lambda}(\tau, V(\tau)) \geq \frac{1}{2} \nu(V(\tau))>\tilde{C}_{0}$ for some $\tilde{C}_{0}>0$, for which we also have

$$
\int_{0}^{t} e^{-\int_{s}^{t} v(V(\tau)) d \tau} v(V(s)) d s<\infty .
$$

We now turn to estimate $\mathcal{H}_{i}(1 \leq i \leq 16)$ individually. We still start with the nonlocal terms $\mathcal{H}_{6}, \mathcal{H}_{8}, \mathcal{H}_{9}, \mathcal{H}_{13}, \mathcal{H}_{14}, \mathcal{H}_{15}$ and $\mathcal{H}_{16}$, which turn out to be more intricate and be different from the corresponding estimates in the proof of Theorem 3.1, because the estimates we want to obtain here must be uniform in time $t \in(0, \infty)$.

For $\mathcal{H}_{6}$, if $\gamma=0$, one gets from Lemma 4.4 that

$$
\left|\mathcal{H}_{6}\right| \leq \frac{C}{M} \int_{0}^{t} e^{-\int_{s}^{t} \frac{v(V(\tau))}{2} d \tau} d s \sup _{0 \leq s \leq t \zeta^{\prime} \leq \zeta} \sum_{l}\left\|w_{l} \partial_{\zeta^{\prime}}^{\vartheta} g_{1}(s)\right\|_{L^{\infty}}
$$




$$
\leq \frac{C}{M} \sup _{0 \leq s \leq t \zeta^{\prime} \leq \zeta}\left\|w_{l} \partial_{\zeta^{\prime}}^{\vartheta} g_{1}(s)\right\|_{L^{\infty}}
$$

If $0<\gamma \leq 1$, by (3.15) and using (4.13) in Lemma 4.6, we have

$$
\begin{aligned}
\left|\mathcal{H}_{6}\right| & \leq C \int_{0}^{t} e^{-\int_{s}^{t} \frac{v(V(\tau))}{2} d \tau} v(V(s)) d s \sup _{0 \leq s \leq t}\left\|\left\{v^{-1} w_{l} \partial_{\zeta}^{\vartheta}\left(\chi_{M} \mathcal{K} g_{1}\right)\right\}(s, X(s), V(s))\right\|_{L^{\infty}} \\
& \leq\left(\mathbf{1}_{\gamma>0} \frac{C}{M^{\frac{\gamma}{2}}}+s\right) \sup _{0 \leq s \leq t \zeta^{\prime} \leq \zeta}\left\|w_{l} \partial_{\zeta^{\prime}}^{\vartheta} g_{1}(s)\right\|_{L^{\infty}} .
\end{aligned}
$$

Next, thanks to Lemma 4.7 and the a priori assumption (3.7) as well as (3.15), it follows

$$
\begin{aligned}
& \left|\mathcal{H}_{8}\right| \leq C \int_{0}^{t} e^{-\int_{s}^{t} \frac{v(V(\tau))}{2} d \tau} v(V(s)) d s \sup _{0 \leq s \leq t} \| e^{\lambda_{0} s}\left\{v ^ { - 1 } w _ { l } \partial _ { \zeta } ^ { \vartheta } \left\{Q\left(f_{1}, f_{1}\right)+Q\left(f_{1}, \sqrt{\mu} f_{2}\right)\right.\right. \\
& \left.\left.+Q\left(\sqrt{\mu} f_{2}, f_{1}\right)\right\}\right\}(s, X(s), V(s)) \|_{L^{\infty}} \\
& \leq C \sup _{0 \leq s \leq t} \sum_{\substack{\zeta^{\prime}+\zeta^{\prime \prime} \leq \zeta \\
\vartheta^{\prime} \leq \vartheta}}\left\{\left\|w_{l} \partial_{\zeta^{\prime}}^{\vartheta^{\prime}} g_{1}(s)\right\|_{L^{\infty}}\left\|w_{l} \partial_{\zeta^{\prime \prime}}^{\vartheta-\vartheta^{\prime}} g_{1}(s)\right\|_{L^{\infty}}\right. \\
& \left.+\left\|w_{l} \partial_{\zeta^{\prime}}^{\vartheta^{\prime}} g_{1}(s)\right\|_{L^{\infty}}\left\|w_{l} \partial_{\zeta^{\prime \prime}}^{\vartheta-\vartheta^{\prime}} g_{2}(s)\right\|_{L^{\infty}}\right\} \\
& \leq C \alpha^{2} \sup _{0 \leq s \leq t \zeta^{\prime} \leq \zeta, \vartheta^{\prime} \leq \vartheta}\left\{\left\|w_{l} \partial_{\zeta^{\prime}}^{\vartheta^{\prime}} g_{1}(s)\right\|_{L^{\infty}}+\left\|w_{l} \partial_{\zeta^{\prime}}^{\vartheta^{\prime}} g_{2}(s)\right\|_{L^{\infty}}\right\}
\end{aligned}
$$

and similarly, in view of (3.15) and Theorem 1.1 and by Lemma 4.7, one has

$$
\begin{aligned}
\left|\mathcal{H}_{9}\right| \leq & C \alpha \sup _{0 \leq s \leq t}\left\|e^{\lambda_{0} s}\left\{v^{-1} w_{l} \partial_{\zeta}^{\vartheta} Q\left(\sqrt{\mu} f, \sqrt{\mu}\left(G_{1}+\alpha G_{R}\right)\right)\right\}(s, X(s), V(s))\right\|_{L^{\infty}} \\
& \left.+C \alpha \sup _{0 \leq s \leq t} \| e^{\lambda_{0} s}\left\{v^{-1} w_{l} \partial_{\zeta}^{\vartheta} Q\left(\sqrt{\mu}\left(G_{1}+\alpha G_{R}\right)\right), \sqrt{\mu} f\right)\right\}(s, X(s), V(s)) \|_{L^{\infty}} \\
\leq & C \alpha \sup _{0 \leq s \leq t \zeta^{\prime} \leq \zeta}\left\|w_{l} \partial_{\zeta^{\prime}}^{\vartheta}\left[g_{1}, g_{2}\right](s)\right\|_{L^{\infty}} \\
\left|\mathcal{H}_{14}\right| \leq & \mathbf{1}_{\zeta>0} C \sup _{0 \leq s \leq t} \| e^{\lambda_{0} s}\left\{v ^ { - 1 } w _ { l } \left\{\partial_{\zeta}^{\vartheta}\left[Q\left(\sqrt{\mu} f_{2}, \mu\right)+Q_{\text {gain }}\left(\mu, \sqrt{\mu} f_{2}\right)\right]\right.\right. \\
- & \left.\left.-\left[Q\left(\sqrt{\mu} \partial_{\zeta}^{\vartheta} f_{2}, \mu\right)+Q_{\text {gain }}\left(\mu, \sqrt{\mu} \partial_{\zeta}^{\vartheta} f_{2}\right)\right]\right\}\right\}(s, V(s)) \|_{L^{\infty}} \\
\leq & \mathbf{1}_{\zeta>0} C \sup _{0 \leq s \leq t \zeta^{\prime}<\zeta}\left\|w_{l} \partial_{\zeta^{\prime}}^{\vartheta} g_{2}(s)\right\|_{L^{\infty \prime}}
\end{aligned}
$$




$$
\begin{aligned}
\left|\mathcal{H}_{15}\right| & \leq C \sup _{0 \leq s \leq t}\left\|e^{\lambda_{0} s}\left\{v^{-1} w_{l} \partial_{\zeta}^{\vartheta}\left\{\left(1-\chi_{M}\right) \mu^{-\frac{1}{2}}\left[Q\left(f_{1}, \mu\right)+Q_{\text {gain }}\left(\mu, f_{1}\right)\right]\right\}\right\}(s, V(s))\right\|_{L^{\infty}} \\
& \leq C \sup _{0 \leq s \leq t \zeta^{\prime} \leq \zeta}\left\|w_{l} \partial_{\zeta^{\prime}}^{\vartheta} g_{1}(s)\right\|_{L^{\infty}} .
\end{aligned}
$$

For $\mathcal{H}_{16}$, in light of Lemma 4.2 and the a priori assumption (3.8), it follows

$$
\begin{aligned}
\left|\mathcal{H}_{16}\right| & \leq C \sup _{0 \leq s \leq t \zeta^{\prime}+\zeta^{\prime \prime} \leq \zeta, \vartheta^{\prime} \leq \vartheta}\left\|w_{l} \partial_{\zeta^{\prime}}^{\vartheta^{\prime}} g_{2}(s)\right\|_{L^{\infty}}\left\|w_{l} \partial_{\zeta^{\prime \prime}}^{\vartheta-\vartheta^{\prime}} g_{2}(s)\right\|_{L^{\infty}} \\
& \leq C \alpha \sup _{0 \leq s \leq t \zeta^{\prime} \leq \zeta, \vartheta^{\prime} \leq \vartheta}\left\|w_{l} \partial_{\zeta^{\prime}}^{\vartheta^{\prime}} g_{2}(s)\right\|_{L^{\infty}} .
\end{aligned}
$$

For the delicate nonlocal term $\mathcal{H}_{13}$, we first rewrite

$$
\mathcal{H}_{13}=\int_{0}^{t} e^{-\int_{s}^{t} \mathcal{A}^{\lambda}(\tau) d \tau} \int_{\mathbb{R}^{3}} \mathbf{k}_{w}\left(V(s), v_{*}\right)\left(w_{l} \partial_{\zeta}^{\vartheta} g_{2}\right)\left(s, X(s), v_{*}\right) d v_{*} d s
$$

As in Section 2, the computation for $\mathcal{H}_{13}$ is then divided into the following three cases.

Case 1. $|V(s)|>M$. In this case, we get from Lemma 4.1 that

$$
\left|\mathcal{H}_{13}\right| \leq \frac{C}{M} \sup _{0 \leq s \leq t}\left\|w_{l} \partial_{\zeta}^{\vartheta} g_{2}(s)\right\|_{L^{\infty}}
$$

Case 2. $|V(s)| \leq M$ and $\left|v_{*}\right|>2 M$. At this stage, one has $\left|V(s)-v_{*}\right|>M$, thus it follows

$$
\mathbf{k}_{w}\left(V, v_{*}\right) \leq C e^{-\frac{\varepsilon M^{2}}{8}} \mathbf{k}_{w}\left(V, v_{*}\right) e^{\frac{\varepsilon\left|V-v_{*}\right|^{2}}{8}},
$$

which gives

$$
\left|\mathcal{H}_{13}\right| \leq C e^{-\frac{\varepsilon M^{2}}{8}} \sup _{0 \leq s \leq t}\left\|w_{l} \partial_{\zeta}^{\vartheta} g_{2}(s)\right\|_{L^{\infty}}
$$

according to Lemma 4.1.

Case 3. $|V(s)| \leq M$ and $\left|v_{*}\right| \leq 2 M$. The key point in this case is to make use of the boundedness of the operator $K$ on the complement of a singular set, so that (3.16) can be controlled by the $L^{1}$ norm of $g_{2}$, which further can be converted to the $L^{2}$ norm. To see this, for any large $M>0$, we choose a number $p(M)$ to introduce $\mathbf{k}_{w, p}\left(V, v_{*}\right)$ as $(2.27)$, and then write

$$
\mathcal{H}_{13}=\int_{0}^{t} e^{-\int_{s}^{t} \mathcal{A}^{\lambda}(\tau) d \tau} \int_{\mathbb{R}^{3}}\left[\mathbf{k}_{w}-\mathbf{k}_{w, p}+\mathbf{k}_{w, p}\right]\left(V(s), v_{*}\right)\left(w_{l} \partial_{\zeta}^{\vartheta} g_{2}\right)\left(s, X(s), v_{*}\right) d v_{*} d s,
$$


which further gives the bound

$$
\begin{aligned}
\left|\mathcal{H}_{13}\right| \leq & \frac{C}{M} \sup _{0 \leq s \leq t}\left\|w_{l} \partial_{\zeta}^{\vartheta} g_{2}(s)\right\|_{L^{\infty}} \\
& +\underbrace{\int_{0}^{t} e^{-\int_{s}^{t} \mathcal{A}^{\lambda}(\tau) d \tau} \mathbf{1}_{|V(s)| \leq M} \int_{\left|v_{*}\right| \leq 2 M} \mathbf{k}_{w, p}\left(V(s), v_{*}\right)\left|\left(w_{l} \partial_{\zeta}^{\vartheta} g_{2}\right)\left(s, X(s), v_{*}\right)\right| d v_{*} d s}_{\mathfrak{I}} .
\end{aligned}
$$

Putting the above estimate for $\mathcal{H}_{13}$ together, we thus have

$$
\left|\mathcal{H}_{13}\right| \leq C\left(e^{-\frac{\varepsilon M^{2}}{8}}+\frac{1}{M}\right) \sup _{0 \leq s \leq t}\left\|w_{l} \partial_{\zeta}^{\vartheta} g_{2}(s)\right\|_{L^{\infty}}+\mathfrak{I}
$$

Up to now, one cannot deduce the desired estimate for $\mathfrak{I}$, which in fact will be handled by iteration argument once all the other terms in the right hand side of (3.14) have been properly controlled.

Let us now turn to compute the other terms in the right hand side of (3.13) and (3.14). It is straightforward to see

$$
\begin{aligned}
& \left|\mathcal{H}_{1}\right| \leq\left\|w_{l} \partial_{\zeta}^{\vartheta} f_{0}\right\|_{L^{\infty \prime}} \\
& \left|\mathcal{H}_{2}\right|,\left|\mathcal{H}_{5}\right| \leq \mathbf{1}_{\zeta>0} C \sup _{0 \leq s \leq t} \sum_{\substack{\zeta^{\prime}<\zeta \\
\zeta^{\prime}+\vartheta^{\prime}=\zeta+\vartheta}}\left\|w_{l} \partial_{\zeta^{\prime}}^{\vartheta^{\prime}} g_{1}(s)\right\|_{L^{\infty}} \\
& \left|\mathcal{H}_{10}\right|,\left|\mathcal{H}_{12}\right| \leq \mathbf{1}_{\zeta>0} C \sup _{0 \leq s \leq t} \sum_{\substack{\zeta^{\prime}<\zeta \\
\zeta^{\prime}+\vartheta^{\prime}=\zeta+\vartheta}}\left\|w_{l} \partial_{\zeta^{\prime}}^{\vartheta^{\prime}} g_{2}(s)\right\|_{L^{\infty}} .
\end{aligned}
$$

From (2.11), it follows $|\beta| \leq C \alpha$. We then have

$$
\left|\mathcal{H}_{3}\right|+\left|\mathcal{H}_{4}\right|+\left|\mathcal{H}_{11}\right| \leq C \alpha \sup _{0 \leq s \leq t}\left\|w_{l} \partial_{\zeta}^{\vartheta}\left[g_{1}, g_{2}\right](s)\right\|_{L^{\infty}}
$$

and

$$
\left|\mathcal{H}_{7}\right| \leq C \alpha \sup _{0 \leq s \leq t \zeta^{\prime} \leq \zeta}\left\|w_{l} \partial_{\zeta^{\prime}}^{\vartheta} g_{2}(s)\right\|_{L^{\infty}}
$$

Consequently, by plugging all the above estimates for $\mathcal{H}_{i}(1 \leq i \leq 16)$ into (3.13) and (3.14), respectively, one gets

$$
\left|w_{l} \partial_{\zeta}^{\vartheta} g_{1}(t, x, v)\right| \leq\left\|w_{l} \partial_{\zeta}^{\vartheta} f_{0}\right\|_{L^{\infty}}+\mathbf{1}_{\zeta>0} C \sup _{0 \leq s \leq t} \sum_{\substack{\zeta^{\prime}<\zeta \\ \zeta^{\prime}+\vartheta^{\prime}=\zeta+\vartheta}}\left\|w_{l} \partial_{\zeta^{\prime}}^{\vartheta^{\prime}} g_{1}(s)\right\|_{L^{\infty}}
$$




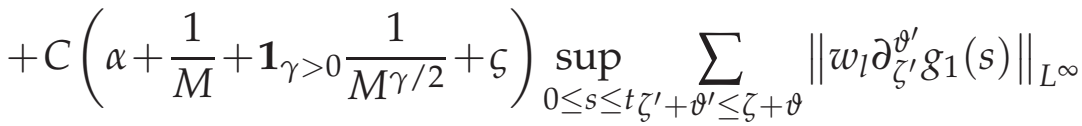

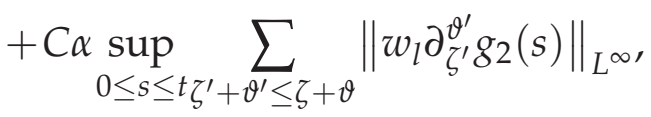

$$
\begin{aligned}
& \left|w_{l} \partial_{\zeta}^{\vartheta} g_{2}(t, x, v)\right| \\
& \leq C \sup _{0 \leq s \leq t} \sum_{\zeta^{\prime} \leq \zeta}\left\|w_{l} \partial_{\zeta^{\prime}}^{\vartheta} g_{1}(s)\right\|_{L^{\infty}}+\mathbf{1}_{\zeta>0} C \sup _{0 \leq s \leq t} \sum_{\substack{\zeta^{\prime}<\zeta \\
\left|\zeta^{\prime}\right|+\left|\vartheta^{\prime}\right|=\zeta+\vartheta}}\left\|w_{l} \partial_{\zeta^{\prime}}^{\vartheta^{\prime}} g_{2}(s)\right\|_{L^{\infty}} \\
& +C\left(\alpha+e^{-\frac{\varepsilon M^{2}}{8}}+\frac{1}{M}\right) \sup _{0 \leq s \leq t \zeta^{\prime} \leq \zeta}\left\|w_{l} \partial_{\zeta^{\prime}}^{\vartheta} g_{2}(s)\right\|_{L^{\infty}}+\mathfrak{I} .
\end{aligned}
$$

To continue, we have by substituting (3.19) into $\mathfrak{I}$ defined in (3.17) that

$$
\begin{aligned}
\mathfrak{I} \leq & C \int_{0}^{t} e^{-\int_{s}^{t} \mathcal{A}^{\lambda}(\tau) d \tau} \mathbf{1}_{|V(s)| \leq M} \int_{\left|v_{*}\right| \leq 2 M} \mathbf{k}_{w, p}\left(V(s), v_{*}\right) \\
& \times\left\{\sup _{0 \leq \tau \leq s} \sum_{\zeta^{\prime} \leq \zeta}\left\|w_{l} \partial_{\zeta^{\prime}}^{\vartheta} g_{1}(\tau)\right\|_{L^{\infty}}+\mathbf{1}_{\zeta>0} \sup _{0 \leq \tau \leq s} \sum_{\substack{\zeta^{\prime}<\zeta \\
\left|\zeta^{\prime}\right|+\left|\vartheta^{\prime}\right|=\zeta+\vartheta}}\left\|w_{l} \partial_{\zeta^{\prime}}^{\vartheta^{\prime}} g_{2}(\tau)\right\|_{L^{\infty}}\right\} d v_{*} d s \\
& +\int_{0}^{t} e^{-\int_{s}^{t} \mathcal{A}^{\lambda}(\tau) d \tau} \mathbf{1}_{|V(s)| \leq M} \int_{\left|v_{*}\right| \leq 2 M} \mathbf{k}_{w, p}\left(V(s), v_{*}\right) \int_{0}^{s} e^{-\int_{s^{\prime}}^{s} \mathcal{A}^{\lambda}(\tau) d \tau} \mathbf{1}_{\left|V\left(s^{\prime}\right)\right| \leq M} \\
& \times \int_{\left|v_{*}^{\prime}\right| \leq 2 M} \mathbf{k}_{w, p}\left(V\left(s^{\prime}\right), v_{*}^{\prime}\right) \mathbf{1}_{X\left(s^{\prime}\right) \in \mathbb{T}^{3} \mid}\left|\left(w_{l} \partial_{\zeta}^{\vartheta} g_{2}\right)\left(s^{\prime}, X\left(s^{\prime}\right), v_{*}^{\prime}\right)\right| d v_{*}^{\prime} d v_{*} d s^{\prime} d s,
\end{aligned}
$$

where we have denoted

$$
\begin{aligned}
& V\left(s^{\prime}\right)=V\left(s^{\prime} ; s, X(s), v_{*}\right)=e^{-\left(s^{\prime}-s\right)(\beta I+\alpha A)} v_{*}, \\
& X\left(s^{\prime}\right)=X\left(s^{\prime} ; s, X(s), v_{*}\right)=X(s)-(\beta I+\alpha A)^{-1}\left[e^{-\left(s^{\prime}-s\right)(\beta I+\alpha A)}-I\right] v_{*},
\end{aligned}
$$

according to (3.12). As a consequence, (3.20) further implies

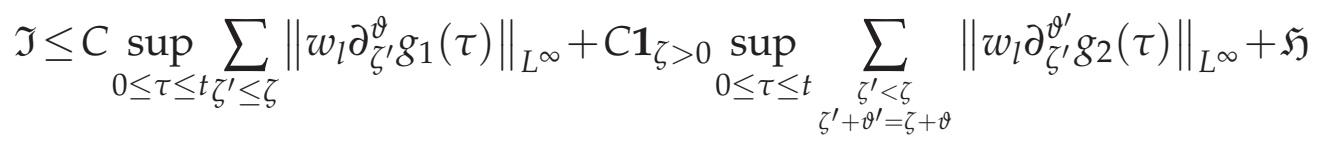

with $\mathfrak{H}$ denoting the second term in the right hand side of (3.20). To compute $\mathfrak{H}$, we then split it into the following two integrals:

$$
\mathfrak{H}=\int_{0}^{t} e^{-\int_{s}^{t} \mathcal{A}^{\lambda}(\tau) d \tau} \mathbf{1}_{|V(s)| \leq M} \int_{\left|v_{*}\right| \leq 2 M} \mathbf{k}_{w, p}\left(V(s), v_{*}\right)\left\{\int_{0}^{s-\eta_{0}}+\int_{s-\eta_{0}}^{s}\right\}
$$




$$
\begin{aligned}
& \times e^{-\int_{s^{\prime}}^{s} \mathcal{A}^{\lambda}(\tau) d \tau} \mathbf{1}_{\left|V\left(s^{\prime}\right)\right| \leq M} \int_{\left|v_{*}^{\prime}\right| \leq 2 M} \mathbf{k}_{w, p}\left(V\left(s^{\prime}\right), v_{*}^{\prime}\right) \mathbf{1}_{X\left(s^{\prime}\right) \in \mathbb{T}^{3}} \\
& \times\left|\left(w_{l} \partial_{\zeta}^{\vartheta} g_{2}\right)\left(s^{\prime}, X\left(s^{\prime}\right), v_{*}^{\prime}\right)\right| d v_{*}^{\prime} d v_{*} d s^{\prime} d s=: \mathfrak{H}_{1}+\mathfrak{H}_{2},
\end{aligned}
$$

where $\eta_{0}>0$ is suitably small. It is straightforward to see that

$$
\mathfrak{H}_{2} \leq C \eta_{0} \sup _{0 \leq s \leq t}\left\|w_{l} \partial_{\zeta}^{\vartheta} g_{2}(s)\right\|_{L^{\infty}} .
$$

For $\mathfrak{H}_{1}$, since $s-s^{\prime} \geq \eta_{0}$ in this integral, the Jacobian

$$
\tilde{\mathcal{J}}:=|| \frac{\partial X\left(s^{\prime}\right)}{\partial v_{*}}||=||(\beta I+\alpha A)^{-1}\left[e^{-\left(s^{\prime}-s\right)(\beta I+\alpha A)}-I\right]|| \geq \frac{\left(s-s^{\prime}\right)^{3}}{8} \geq \frac{\eta_{0}^{3}}{8},
$$

according to Lemma 4.8. Moreover, if we denote

$$
\Omega_{y}=\left\{y|| y-X(s)|\leq|(\beta I+\alpha A)^{-1}\left[e^{-\left(s^{\prime}-s\right)(\beta I+\alpha A)}-I\right] v_{*} \mid\right\},
$$

then, by applying (4.15) of Lemma 4.8, we have

$$
\left|\Omega_{y}\right| \leq C\left(s-s^{\prime}\right) e^{C \alpha\left(s-s^{\prime}\right)} .
$$

With these, one gets by a change of variable $X\left(s^{\prime}\right) \rightarrow y$ that

$$
\begin{aligned}
\mathfrak{H}_{2} & \leq C \int_{0}^{t} \mathcal{B} \int_{0}^{s-\eta_{0}} \mathcal{D} \int_{\left|v_{*}^{\prime}\right| \leq 2 M}\left(\int_{\left|v_{*}\right| \leq 2 M}\left|\left(\partial_{\zeta}^{\vartheta} g_{2}\right)\left(s^{\prime}, X\left(s^{\prime}\right), v_{*}^{\prime}\right)\right|^{2} d v_{*}\right)^{\frac{1}{2}} d v_{*}^{\prime} d s^{\prime} d s \\
& \leq C \int_{0}^{t} \mathcal{B} \int_{0}^{s-\eta_{0}} \mathcal{D} \int_{\left|v_{*}^{\prime}\right| \leq 2 M}\left(\int_{\Omega_{y}} \tilde{\mathcal{J}}^{-3}\left|\left(\partial_{\zeta}^{\vartheta} g_{2}\right)\left(s^{\prime}, y, v_{*}^{\prime}\right)\right|^{2} d y\right)^{\frac{1}{2}} d v_{*}^{\prime} d s^{\prime} d s \\
& \leq C \eta_{0}^{-\frac{3}{2}} \int_{0}^{t} \mathcal{B} \int_{0}^{s-\eta_{0}} \mathcal{D}\left(\left|\Omega_{y}\right|^{\frac{1}{2}}+1\right) \int_{\left|v_{*}^{\prime}\right| \leq 2 M}\left(\int_{\mathbb{T}^{3}}\left|\left(\partial_{\zeta}^{\vartheta} g_{2}\right)\left(s^{\prime}, y, v_{*}^{\prime}\right)\right|^{2} d y\right)^{\frac{1}{2}} d v_{*}^{\prime} d s^{\prime} d s \\
& \leq C \eta_{0}^{-\frac{3}{2}} \sup _{0 \leq s \leq t}\left(\int_{\mathbb{R}^{3}} \int_{\mathbb{T}^{3}}\left|\left(\partial_{\zeta}^{\vartheta} g_{2}\right)(s, y, v)\right|^{2} d y d v\right)^{\frac{1}{2}},
\end{aligned}
$$

where $\mathcal{B}=\mathcal{B}(t, s)=e^{-c_{0}(t-s)}$ and $\mathcal{D}=\mathcal{D}\left(s, s^{\prime}\right)=e^{-c_{0}\left(s-s^{\prime}\right)}$. Thus, it follows

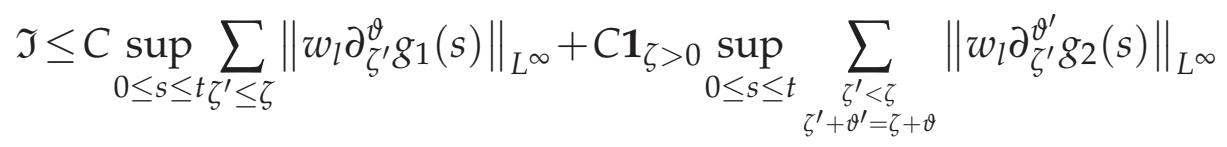




$$
+C \eta_{0} \sup _{0 \leq s \leq t}\left\|w_{l} \partial_{\zeta}^{\vartheta} g_{2}(s)\right\|_{L^{\infty}}+C_{\eta_{0}} \sup _{0 \leq s \leq t}\left\|\partial_{\zeta}^{\vartheta} g_{2}(s)\right\|
$$

This together with (3.19) further gives

$$
\begin{aligned}
\left|w_{l} \partial_{\zeta}^{\vartheta} g_{2}(t, x, v)\right| \leq C & \sup _{0 \leq s \leq t \zeta^{\prime} \leq \zeta} \sum_{l}\left\|w_{l} \partial_{\zeta^{\prime}}^{\vartheta} g_{1}(s)\right\|_{L^{\infty}}+\mathbf{1}_{\zeta>0} C \sup _{0 \leq s \leq t} \sum_{\substack{\zeta^{\prime}<\zeta \\
\zeta^{\prime}+\vartheta^{\prime}=\zeta+\vartheta}}\left\|w_{l} \partial_{\zeta^{\prime}}^{\vartheta^{\prime}} g_{2}(s)\right\|_{L^{\infty}} \\
& +C\left(\alpha+e^{-\frac{\varepsilon M^{2}}{8}}+\frac{1}{M}+\eta_{0}\right) \sup _{0 \leq s \leq t \zeta^{\prime} \leq \zeta} \sum_{0}\left\|w_{l} \partial_{\zeta^{\prime}}^{\vartheta} g_{2}(s)\right\|_{L^{\infty}} \\
& +C_{\eta_{0}} \sup _{0 \leq s \leq t}\left\|\partial_{\zeta}^{\vartheta} g_{2}(s)\right\| .
\end{aligned}
$$

Finally, taking a linear combination of (3.18) and (3.21) with $|\zeta|=0,1, \ldots, N$ and $|\zeta|+|\vartheta| \leq N$, respectively, and adjusting constants, we conclude

$$
\begin{aligned}
& \sup _{0 \leq s \leq t|\zeta|+|\vartheta| \leq N} \sum_{0}\left\|w_{l} \partial_{\zeta}^{\vartheta} g_{1}(s)\right\|_{L^{\infty}} \\
\leq & \sum_{|\zeta|+|\vartheta| \leq N}\left\|w_{l} \partial_{\zeta}^{\vartheta} f_{0}\right\|_{L^{\infty}}+C \alpha \sup _{0 \leq s \leq t} \sum_{|\zeta|+|\vartheta| \leq N}\left\|w_{l} \partial_{\zeta}^{\vartheta} g_{2}(s)\right\|_{L^{\infty}} \\
& \sup _{0 \leq s \leq t|\zeta|+|\vartheta| \leq N}\left\|w_{l} \partial_{\zeta}^{\vartheta} g_{2}(s)\right\|_{L^{\infty}} \\
\leq & C \sup _{0 \leq s \leq t} \sum_{|\zeta|+|\vartheta| \leq N}\left\|w_{l} \partial_{\zeta}^{\vartheta} g_{1}(s)\right\|_{L^{\infty}}+C \sup _{0 \leq s \leq t|\zeta|+|\vartheta| \leq N} \sum_{0}\left\|\partial_{\zeta}^{\vartheta} g_{2}(s)\right\| .
\end{aligned}
$$

Actually, from the proof presented above, we have the following refined estimates concerning the $L^{\infty}$ norm of $g_{1}$ and $g_{2}$ without velocity derivatives.

Lemma 3.1. Under the hypothesis (3.8), it holds that

$$
\begin{aligned}
& \sup _{0 \leq s \leq t}\left\|w_{l} g_{1}(s)\right\|_{L^{\infty}} \leq C\left\|w_{l} f_{0}\right\|_{L^{\infty}}+C \alpha \sup _{0 \leq s \leq t}\left\|w_{l} g_{2}(s)\right\|_{L^{\infty}}, \\
& \sup _{0 \leq s \leq t}\left\|w_{l} g_{2}(s)\right\|_{L^{\infty}} \leq C \sup _{0 \leq s \leq t}\left\|w_{l} g_{1}(s)\right\|_{L^{\infty}}+C \sup _{0 \leq s \leq t}\left\|g_{2}(s)\right\|,
\end{aligned}
$$

and

$$
\begin{aligned}
& \sup _{0 \leq s \leq t} \sum_{1 \leq|\vartheta| \leq N}\left\|w_{l} \partial^{\vartheta} g_{1}(s)\right\|_{L^{\infty}} \\
& \leq C \sum_{1 \leq|\vartheta| \leq N}\left\|w_{l} \partial^{\vartheta} f_{0}\right\|_{L^{\infty}}+C \alpha \sup _{0 \leq s \leq t} \sum_{1 \leq|\vartheta| \leq N}\left\|w_{l} \partial^{\vartheta} g_{2}(s)\right\|_{L^{\infty}},
\end{aligned}
$$




$$
\begin{aligned}
& \sup _{0 \leq s \leq t} \sum_{1 \leq|\vartheta| \leq N}\left\|w_{l} \partial^{\vartheta} g_{2}(s)\right\|_{L^{\infty}} \\
\leq C & \sup _{0 \leq s \leq t} \sum_{1 \leq|\vartheta| \leq N}\left\|w_{l} \partial^{\vartheta} g_{1}(s)\right\|_{L^{\infty}}+C \sup _{0 \leq s \leq t} \sum_{1 \leq|\vartheta| \leq N}\left\|\partial^{\vartheta} g_{2}(s)\right\| .
\end{aligned}
$$

Step 2. $L^{2}$ estimates. To close our final estimate, it remains then to deduce the $H_{x, v}^{N}$ estimate of $e^{\lambda_{0} t} f_{2}(t, v)$ in (3.23). The computation is divided into the following three sub-steps.

Step 2.1. The estimates for $c$. In this sub-step, we consider the basic $L^{2}$ estimate for $c$, which is difficult to be obtained due to the exponential growth of the heat flux, cf. [25]. Recall $\left[g_{1}, g_{2}\right](t, v)=e^{\lambda_{0} t}\left[f_{1}, f_{2}\right](t, v)$ and $\sqrt{\mu} g=g_{1}+\sqrt{\mu} g_{2}$. It is straightforward to see that $g$ satisfies

$$
\begin{aligned}
& \partial_{t} g+v \cdot \nabla_{x} g-\beta \mu^{-\frac{1}{2}} \nabla_{v} \cdot(v \sqrt{\mu} g)-\alpha \mu^{-\frac{1}{2}} \nabla_{v} \cdot(A v \sqrt{\mu} g)-\lambda_{0} g+L g \\
& =\underbrace{e^{-\lambda_{0} t} \Gamma(g, g)+\alpha\left\{\Gamma\left(G_{1}+\alpha G_{R}, g\right)+\Gamma\left(g, G_{1}+\alpha G_{R}\right)\right\}}_{\Re}
\end{aligned}
$$

with

$$
\sqrt{\mu} g(0, x, v)=f_{0}(x, v)
$$

Similar to (2.53), we define

$$
\begin{aligned}
& \mathbf{P}_{0} g=\left\{a(t, x)+\mathbf{b}(t, x) \cdot v+c(t, x)\left(|v|^{2}-3\right)\right\} \sqrt{\mu}, \\
& \overline{\mathbf{P}}_{0} g_{1}=\left\{a_{1}(t, x)+\mathbf{b}_{1}(t, x) \cdot v+c_{1}(t, x)\left(|v|^{2}-3\right)\right\} \mu,
\end{aligned}
$$

and recall the definition

$$
\mathbf{P}_{0 g_{2}}=\left\{a_{2}(t, x)+\mathbf{b}_{2}(t, x) \cdot v+c_{2}(t, x)\left(|v|^{2}-3\right)\right\} \sqrt{\mu} .
$$

Then it follows

$$
\begin{aligned}
& a(t, x)=a_{1}(t, x)+a_{2}(t, x), \\
& \mathbf{b}(t, x)=\mathbf{b}_{1}(t, x)+\mathbf{b}_{2}(t, x), \\
& c(t, x)=c_{1}(t, x)+c_{2}(t, x)
\end{aligned}
$$

for any $t \geq 0$ and $x \in \mathbb{T}^{3}$. In addition, (3.26) together with (1.15) and (3.27) implies

$$
\int_{\mathbb{T}^{3}} a(t, x) d x=0, \quad \int_{\mathbb{T}^{3}} b_{i}(t, x) d x=0, \quad i=1,2,3,
$$


where we have denoted $\mathbf{b}=\left(b_{1}, b_{2}, b_{3}\right)$. Next, taking the moments

$$
\sqrt{\mu}, \quad v_{i} \sqrt{\mu}, \quad \frac{1}{6}\left(|v|^{2}-3\right) \sqrt{\mu}, \quad i=1,2,3
$$

for Eq. (3.26), one has

$$
\begin{aligned}
& \partial_{t} a+\nabla_{x} \cdot \mathbf{b}=0, \\
& \partial_{t} b_{i}+\partial_{i}(a+2 c)+\sum_{j}\left(\left(\beta-\lambda_{0}\right) I+\alpha A\right)_{i j} b_{j}+\sum_{j} \partial_{j}\left\langle\mathscr{A}_{i j}, \mathbf{P}_{1} g\right\rangle=0, \\
& \partial_{t} c+\frac{1}{3} \nabla_{x} \cdot \mathbf{b}+\beta_{1} \alpha^{2}(2 c+a)-\lambda_{0} c+\frac{1}{6} \nabla_{x} \cdot\left\langle\left(|v|^{2}-5\right) v \sqrt{\mu}, \mathbf{P}_{1} g\right\rangle \\
& \quad+\frac{\alpha}{3} \sum_{i, j} a_{i j}\left\langle\mathscr{A}_{i j}, \mathbf{P}_{1} g\right\rangle=0
\end{aligned}
$$

where

$$
\mathscr{A}_{i j}=\left(v_{i} v_{j}-\frac{\delta_{i j}}{3}|v|^{2}\right) \sqrt{\mu}, \quad i, j=1,2,3
$$

with $\delta_{i j}$ being the Kronecker delta, and the identity $\beta_{0}+\frac{1}{3} \operatorname{tr} A=0$ was used while deriving (3.31).

Furthermore, taking the higher order moments $\mathscr{A}_{i j}$ and

$$
\mathscr{B}_{i} \stackrel{\text { def }}{=} \frac{1}{10}\left(|v|^{2}-5\right) v_{i} \sqrt{\mu}, \quad i, j=1,2,3
$$

for Eq. (3.26), respectively, we obtain

$$
\begin{aligned}
\partial_{t} & \left\langle\mathscr{A}_{i j}, \mathbf{P}_{1} g\right\rangle+\partial_{i} b_{j}+\partial_{j} b_{i}-\frac{2}{3} \nabla_{x} \cdot \mathbf{b} \delta_{i j}+\left\langle\mathscr{A}_{i j}, v \cdot \nabla_{x} \mathbf{P}_{1} g\right\rangle \\
& -\beta\left\langle\mu^{-\frac{1}{2}} \nabla_{v} \cdot(v \sqrt{\mu} g), \mathscr{A}_{i j}\right\rangle \\
& \quad-\alpha\left\langle\mu^{-\frac{1}{2}} \nabla_{v} \cdot(A v \sqrt{\mu} g), \mathscr{A}_{i j}\right\rangle-\lambda_{0}\left\langle\mathbf{P}_{1} g, \mathscr{A}_{i j}\right\rangle \\
= & \left\langle-L g+\Re, \mathscr{A}_{i j}\right\rangle, \\
& \partial_{t}\left\langle\mathscr{B}_{i}, \mathbf{P}_{1} g\right\rangle+\partial_{i} c+\left\langle\mathscr{B}_{i}, v \cdot \nabla_{x} \mathbf{P}_{1} g\right\rangle-\beta\left\langle\mu^{-\frac{1}{2}} \nabla_{v} \cdot(v \sqrt{\mu} g), \mathscr{B}_{i}\right\rangle \\
& \quad-\alpha\left\langle\mu^{-\frac{1}{2}} \nabla_{v} \cdot(A v \sqrt{\mu} g), \mathscr{B}_{i}\right\rangle-\lambda_{0}\left\langle\mathbf{P}_{1} g, \mathscr{B}_{i}\right\rangle \\
= & \left\langle-L g+\mathfrak{R}, \mathscr{B}_{i}\right\rangle .
\end{aligned}
$$


Choosing $\lambda_{0}=\beta_{1} \alpha^{2}$, we get from the inner product $((3.31), c)$ that

$$
\begin{aligned}
\frac{1}{2} \frac{d}{d t}\|c\|^{2}+\beta_{1} \alpha^{2}\|c\|^{2}+\frac{\alpha}{3} \sum_{i, j} a_{i j}\left(c,\left\langle\mathscr{A}_{i j}, \mathbf{P}_{1} g_{1}\right\rangle\right)+\frac{\alpha}{3} \sum_{i, j} a_{i j}\left(c_{1},\left\langle\mathscr{A}_{i j}, \mathbf{P}_{1} g_{2}\right\rangle\right) \\
+\frac{\alpha}{3} \sum_{i, j} a_{i j}\left(c_{2},\left\langle\mathscr{A}_{i j}, \mathbf{P}_{1} g_{2}\right\rangle\right)-\frac{1}{3}\left(\mathbf{b}, \nabla_{x} c\right)+\beta_{1} \alpha^{2}(a, c) \\
-\frac{1}{6}\left(\left\langle\left(|v|^{2}-5\right) v \sqrt{\mu}, \mathbf{P}_{1} g\right\rangle, \nabla_{x} c\right)=0 .
\end{aligned}
$$

Note that the delicate term $\frac{\alpha}{3} \sum_{i, j} a_{i j}\left(c_{2},\left\langle\mathscr{A}_{i j}, \mathbf{P}_{1} g_{2}\right\rangle\right)$ will be cancelled later on.

We now derive the $L^{2}$ estimate on $\mathbf{P}_{1} g_{2}$. Recall that $g_{2}$ satisfies

$$
\begin{aligned}
& \partial_{t} g_{2}+v \cdot \nabla_{x} g_{2}-\beta \nabla_{v} \cdot\left(v g_{2}\right)-\alpha \nabla_{v} \cdot\left(A v g_{2}\right) \\
& +\alpha A v \cdot\left(\nabla_{v} \sqrt{\mu}\right)\left(|v|^{2}-3\right) c_{2}-\lambda_{0} g_{2}+L g_{2} \\
= & \left(1-\chi_{M}\right) \mu^{-\frac{1}{2}} \mathcal{K} g_{1}+e^{-\lambda_{0} t} \Gamma\left(g_{2}, g_{2}\right),
\end{aligned}
$$

and

$$
g_{2}(0, x, v)=0 .
$$

Taking the inner product of (3.35) and $\mathbf{P}_{1} g_{2}$ over $(x, v) \in \mathbb{T}^{3} \times \mathbb{R}^{3}$ and applying Cauchy-Schwarz's inequality, one has

$$
\begin{aligned}
& \frac{1}{2} \frac{d}{d t}\left\|\mathbf{P}_{1} g_{2}\right\|^{2}-\alpha\left(A v \cdot \nabla_{v}\left\{\left[a_{2}+\mathbf{b}_{2} \cdot v\right] \sqrt{\mu}\right\}, \mathbf{P}_{1} g_{2}\right) \\
& \quad-2 \alpha\left(A v \cdot v \sqrt{\mu} c_{2}, \mathbf{P}_{1} g_{2}\right)+\lambda\left\|\mathbf{P}_{1} g_{2}\right\|_{v}^{2} \\
\leq & C\left\|\nabla_{x}\left[a_{2}, \mathbf{b}_{2}, c_{2}\right]\right\|^{2}+C \alpha^{2}\left\|w_{l} g_{2}\right\|_{L^{\infty}}^{2}+C\left\|w_{l} g_{1}\right\|_{\infty}^{2} \\
\leq & C\left\|\nabla_{x}[a, \mathbf{b}, c]\right\|^{2}+C \alpha^{2}\left\|w_{l} g_{2}\right\|_{L^{\infty}}^{2}+C\left\|w_{l} g_{1}\right\|_{\infty}^{2},
\end{aligned}
$$

according to (3.28), (3.29) and the following estimate:

$$
\begin{aligned}
& \left|\left(\Gamma\left(g_{2}, g_{2}\right), \mathbf{P}_{1} g_{2}\right)\right| \\
\leq & \eta\left\|\mathbf{P}_{1} g_{2}\right\|_{v}^{2}+C_{\eta} \int_{\mathbb{T}^{3}}\left\|v^{\frac{1}{2}} g_{2}\right\|_{L_{v}^{2}}^{4} d x \leq \eta\left\|\mathbf{P}_{1} g_{2}\right\|_{v}^{2}+C_{\eta} \alpha^{2}\left\|w_{l} g_{2}\right\|_{L^{\infty}}^{2}
\end{aligned}
$$

in the case of $l \geq 2$.

Notice that

$$
\left(A v \cdot v \sqrt{\mu} c_{2}, \mathbf{P}_{1} g_{2}\right)=\sum_{i, j} a_{i j}\left(c_{2},\left\langle\mathscr{A}_{i j}, \mathbf{P}_{1} g_{2}\right\rangle\right)
$$


we now get from the summation of (3.34) and $\frac{1}{6}(3.36)$ that

$$
\begin{aligned}
& \frac{1}{2} \frac{d}{d t}\|c\|^{2}+\frac{1}{12} \frac{d}{d t}\left\|\mathbf{P}_{1} g_{2}\right\|^{2}+\lambda \alpha^{2}\|c\|^{2}+\lambda\left\|\mathbf{P}_{1} g_{2}\right\|_{v}^{2} \\
\leq & C_{\eta} \sup _{0 \leq s \leq t}\left\|\nabla_{x}[a, \mathbf{b}, c](s)\right\|^{2}+\left(C \alpha^{2}+\eta\right)\left\|w_{l} g_{2}\right\|_{L^{\infty}}^{2}+C \sup _{0 \leq s \leq t}\left\|w_{l} g_{1}(s)\right\|_{\infty}^{2},
\end{aligned}
$$

where we have used the relations

$$
\left\|g_{1}\right\| \leq\left\|\mathbf{P}_{0} g_{2}(s)\right\|+\left\|\mathbf{P}_{1} g_{2}(s)\right\|, \quad\left\|\mathbf{P}_{0} g_{2}(s)\right\| \leq C\|[a, \mathbf{b}, c]\|+\left\|w_{l} g_{1}(s)\right\|_{L^{\infty}}
$$

for $l>\frac{5}{2}$ according to (3.28). Further, (3.38) gives

$$
\begin{aligned}
\sup _{0 \leq s \leq t} \alpha^{2}\left\|g_{2}(s)\right\|^{2} \leq & C \sup _{0 \leq s \leq t}\left\|\nabla_{x}[a, \mathbf{b}, c](s)\right\|^{2}+\left(C \alpha^{2}+\eta\right) \sup _{0 \leq s \leq t}\left\|w_{l} g_{2}(s)\right\|_{L^{\infty}}^{2} \\
& +C \sup _{0 \leq s \leq t}\left\|w_{l} g_{1}(s)\right\|_{\infty}^{2}
\end{aligned}
$$

Next, substituting (3.39) into (3.24b), one has

$$
\begin{aligned}
\alpha \sup _{0 \leq s \leq t}\left\|w_{l} g_{2}(s)\right\|_{L^{\infty}} \leq & C\left(\alpha+(\eta+\alpha)^{\frac{1}{2}}\right) \sup _{0 \leq s \leq t}\left\|w_{l} g_{1}(s)\right\|_{L^{\infty}} \\
& +C \sup _{0 \leq s \leq t}\left\|\nabla_{x}[a, \mathbf{b}, c](s)\right\| .
\end{aligned}
$$

Finally, we get from (3.24a) and (3.40) that

$$
\begin{aligned}
& \sup _{0 \leq s \leq t}\left\|w_{l} g_{1}(s)\right\|_{L^{\infty}+\alpha \sup _{0 \leq s \leq t}\left\|w_{l} g_{2}(s)\right\|_{L^{\infty}}} \leq C\left\|w_{l} f_{0}\right\|_{L^{\infty}}+C \sup _{0 \leq s \leq t}\left\|\nabla_{x}[a, \mathbf{b}, c](s)\right\| .
\end{aligned}
$$

Step 2.2. Higher order estimates for $[a, \mathbf{b}, c]$. We are now in a position to deduce the higher order $L^{2}$ estimates on $[a, \mathbf{b}, c]$. To do this, we first get from (3.32) that

$$
\sum_{i, i \neq j} \partial_{t} \partial_{i}\left\langle\mathscr{A}_{i j}, \mathbf{P}_{1} g\right\rangle+\partial_{t} \partial_{j}\left\langle\mathscr{A}_{j j}, \mathbf{P}_{1} g\right\rangle+\Delta b_{j}+\frac{1}{3} \partial_{j} \nabla \cdot \mathbf{b}=\mathfrak{T}
$$

with

$$
\mathfrak{T}=\sum_{i, i \neq j} \partial_{i}\left\{-\left\langle\mathscr{A}_{i j}, v \cdot \nabla_{x} \mathbf{P}_{1} g\right\rangle+\beta\left\langle\mu^{-\frac{1}{2}} \nabla_{v} \cdot(v \sqrt{\mu} g), \mathscr{A}_{i j}\right\rangle\right.
$$




$$
\begin{aligned}
& \left.+\alpha\left\langle\mu^{-\frac{1}{2}} \nabla_{v} \cdot(A v \sqrt{\mu} g), \mathscr{A}_{i j}\right\rangle+\lambda_{0}\left\langle\mathbf{P}_{1} g, \mathscr{A}_{i j}\right\rangle+\left\langle-L g+\mathfrak{R}, \mathscr{A}_{i j}\right\rangle\right\} \\
+\partial_{j}\{- & \left\langle\mathscr{A}_{i j}, v \cdot \nabla_{x} \mathbf{P}_{1} g\right\rangle+\beta\left\langle\mu^{-\frac{1}{2}} \nabla_{v} \cdot(v \sqrt{\mu} g), \mathscr{A}_{i j}\right\rangle \\
& \left.+\alpha\left\langle\mu^{-\frac{1}{2}} \nabla_{v} \cdot(A v \sqrt{\mu} g), \mathscr{A}_{i j}\right\rangle+\lambda_{0}\left\langle\mathbf{P}_{1} g, \mathscr{A}_{i j}\right\rangle+\left\langle-L g+\mathfrak{R}, \mathscr{A}_{i j}\right\rangle\right\} .
\end{aligned}
$$

Letting $N-1 \geq|\vartheta| \geq 1$, one has from $\sum_{j}\left(\partial^{\vartheta}(3.42), \partial^{\vartheta} b_{j}\right), \sum_{i}\left(\partial^{\vartheta}(3.33), \partial^{\vartheta} \partial_{i} c\right)$ and $\sum_{i}\left(\partial^{\vartheta}(3.30), \partial^{\vartheta} \partial_{i} a\right)$ that

$$
\begin{aligned}
& \frac{d}{d t} \mathcal{E}_{\mathbf{b}}^{i n t}+\left\|\nabla_{x} \partial^{\vartheta} \mathbf{b}\right\|^{2}+\frac{1}{3}\left\|\partial^{\vartheta} \nabla_{x} \cdot \mathbf{b}\right\|^{2} \\
& =\sum_{j}\left(\sum_{i, i \neq j} \partial_{i} \partial^{\vartheta}\left\langle\mathscr{A}_{i j}, \mathbf{P}_{1} g\right\rangle+\partial_{j} \partial^{\vartheta}\left\langle\mathscr{A}_{j j}, \mathbf{P}_{1} g\right\rangle\right. \text {, } \\
& \left.\partial^{\vartheta}\left(\partial_{j}(a+2 c)+\sum_{i}\left(\left(\beta-\lambda_{0}\right) I+\alpha A\right)_{i j} b_{i}+\sum_{i} \partial_{i}\left\langle\mathscr{A}_{i j}, \mathbf{P}_{1} g\right\rangle\right)\right)-\sum_{j}\left(\partial^{\vartheta} \mathfrak{T}, \partial^{\vartheta} b_{j}\right) \\
& \leq(\eta+\alpha)\left\|\partial^{\vartheta}[a, \mathbf{b}, c]\right\|^{2}+C\left(\epsilon_{0}^{2}+\alpha^{2}\right) \sum_{1 \leq\left|\vartheta^{\prime}\right| \leq N}\left\|w_{l} \partial^{\vartheta^{\prime}} g_{2}\right\|_{L^{\infty}}^{2} \\
& +C_{\eta} \sum_{1 \leq\left|\vartheta^{\prime}\right| \leq N}\left\{\left\|\partial^{\vartheta^{\prime}} \mathbf{P}_{1} g_{2}\right\|^{2}+\left\|w_{l} \partial^{\vartheta^{\prime}} g_{1}\right\|_{L^{\infty}}^{2}\right\} \\
& \frac{d}{d t} \mathcal{E}_{c}^{i n t}+\left\|\nabla_{x} \partial^{\vartheta} c\right\|^{2} \\
& =-\sum_{i}\left(\partial ^ { \vartheta } \left(\left\langle\mathscr{B}_{i}, v \cdot \nabla_{x} \mathbf{P}_{1} g\right\rangle-\beta\left\langle\mu^{-\frac{1}{2}} \nabla_{v} \cdot(v \sqrt{\mu} g), \mathscr{B}_{i}\right\rangle\right.\right. \\
& \left.\left.-\alpha\left\langle\mu^{-\frac{1}{2}} \nabla_{v} \cdot(A v \sqrt{\mu} g), \mathscr{B}_{i}\right\rangle-\lambda_{0}\left\langle\mathbf{P}_{1} g, \mathscr{B}_{i}\right\rangle-\left\langle L g+\mathfrak{R}, \mathscr{B}_{i}\right\rangle\right), \partial^{\vartheta} \partial_{i} c\right) \\
& +\sum_{i}\left(\partial_{i} \partial^{\vartheta}\left\langle\mathscr{B}_{i}, \mathbf{P}_{1} g\right\rangle, \partial^{\vartheta}\left(\beta_{1} \alpha^{2}(2 c+a)-\lambda_{0} c+\frac{1}{6} \nabla_{x} \cdot\left\langle\left(|v|^{2}-5\right) v, \mathbf{P}_{1} g\right\rangle\right.\right. \\
& \left.\left.+\frac{\alpha}{3} \sum_{i, j} a_{i j}\left\langle\mathscr{A}_{i j}, \mathbf{P}_{1} g\right\rangle\right)\right) \\
& \leq(\eta+\alpha)\left\|\partial^{\vartheta}[a, \mathbf{b}, c]\right\|^{2}+C\left(\epsilon_{0}^{2}+\alpha^{2}\right) \sum_{1 \leq\left|\vartheta^{\prime}\right| \leq N}\left\|w_{l} \partial^{\vartheta^{\prime}} g_{2}\right\|_{L^{\infty}}^{2} \\
& +C_{\eta} \sum_{1 \leq\left|\vartheta^{\prime}\right| \leq N}\left\{\left\|\partial^{\vartheta^{\prime}} \mathbf{P}_{1} g_{2}\right\|^{2}+\left\|w_{l} \partial^{\vartheta^{\prime}} g_{1}\right\|_{L^{\infty}}^{2}\right\},
\end{aligned}
$$




$$
\begin{aligned}
& \frac{d}{d t} \mathcal{E}_{a}^{i n t}+\left\|\nabla_{x} \partial^{\vartheta} a\right\|^{2} \\
= & -\sum_{i}\left(\partial^{\vartheta} \partial_{i} b_{i}, \partial^{\vartheta} \nabla_{x} \cdot \mathbf{b}\right)-2 \sum_{i}\left(\partial^{\vartheta} \partial_{i} c, \partial^{\vartheta} \partial_{i} a\right) \\
& -\sum_{i, j}\left(\left[\partial^{\vartheta}\left(\left(\beta-\lambda_{0}\right) I+\alpha A\right)_{i j} b_{j}+\sum_{j} \partial_{j}\left\langle\mathscr{A}_{i j}, \mathbf{P}_{1} g\right\rangle\right], \partial^{\vartheta} \partial_{i} a\right) \\
\leq & (\eta+\alpha)\left\|\left[\partial^{\vartheta} a, \nabla_{x} \partial^{\vartheta} a\right]\right\|^{2}+C\left\|\nabla_{x} \partial^{\vartheta}[\mathbf{b}, c]\right\|^{2} \\
& +C_{\eta} \sum_{1 \leq\left|\vartheta^{\prime}\right| \leq N}\left\{\left\|\partial^{\vartheta^{\prime}} \mathbf{P}_{1} g_{2}\right\|^{2}+\left\|w_{l} \partial^{\vartheta^{\prime}} g_{1}\right\|_{L^{\infty}}^{2}\right\},
\end{aligned}
$$

respectively, where we have set

$$
\begin{aligned}
& \mathcal{E}_{\mathbf{b}}^{i n t}=-\sum_{j}\left(\partial^{\vartheta}\left(\sum_{i, i \neq j} \partial_{i}\left\langle\mathscr{A}_{i j}, \mathbf{P}_{1} g\right\rangle+\partial_{j}\left\langle\mathscr{A}_{j j}, \mathbf{P}_{1} g\right\rangle\right), \partial^{\vartheta} b_{j}\right), \\
& \mathcal{E}_{c}^{i n t}=\sum_{i}\left(\partial^{\vartheta}\left\langle\mathscr{B}_{i}, \mathbf{P}_{1} g\right\rangle, \partial^{\vartheta} \partial_{i} c\right), \\
& \mathcal{E}_{a}^{\text {int }}=\sum_{i}\left(\partial^{\vartheta} \partial_{i} b_{i}, \partial^{\vartheta} \partial_{i} a\right),
\end{aligned}
$$

and in addition, for $l>4$, the following estimates of the type:

$$
\begin{aligned}
& \left\|\partial^{\vartheta}\left\langle L g, \mathscr{B}_{i}\right\rangle\right\|^{2} \\
\leq & C\left\|v^{-1} \Gamma\left(\partial^{\vartheta} \mathbf{P}_{1} g, \sqrt{\mu}\right)\right\|^{2}+C\left\|v^{-1} \Gamma\left(\sqrt{\mu}, \partial^{\vartheta} \mathbf{P}_{1} g\right)\right\|^{2} \\
\leq & C\left\|\mathbf{P}_{1} \partial^{\vartheta} g_{2}\right\|^{2}+C\left\|w_{l} \partial^{\vartheta} g_{2}\right\|_{L^{\infty}}^{2} \\
& \left\|\partial^{\vartheta}\left\langle\Gamma(g, g), \mathscr{B}_{i}\right\rangle\right\|^{2} \\
\leq & C \sum_{\vartheta^{\prime} \leq \vartheta} \int_{\mathbb{T}^{3}}\left\|v^{-1} w_{l} Q\left(\sqrt{\mu} \partial^{\vartheta^{\prime}} g, \sqrt{\mu} \partial^{\vartheta-\vartheta^{\prime}} g\right)\right\|_{L^{\infty}}^{2} d x \\
\leq & C \sum_{\vartheta^{\prime} \leq \vartheta}\left\|w_{l}\left[\partial^{\vartheta^{\prime}} g_{1}, \partial^{\vartheta-\vartheta^{\prime}} g_{2}\right]\right\|_{L^{\infty}}^{4} \leq C \epsilon_{0}^{2} \sum_{1 \leq\left|\vartheta^{\prime}\right| \leq \vartheta}\left\|w_{l}\left[\partial^{\vartheta^{\prime}} g_{1}, \partial^{\vartheta^{\prime}} g_{2}\right]\right\|_{L^{\infty}}^{2}
\end{aligned}
$$

have been used.

Consequently, letting $\kappa_{1}>0$ be suitably small, we get from the summation of (3.43), (3.44) and $\kappa_{1} \times(3.44)$ that

$$
\frac{d}{d t}\left[\kappa_{1} \mathcal{E}_{a}^{i n t}+\mathcal{E}_{\mathbf{b}}^{i n t}+\mathcal{E}_{c}^{i n t}\right]+\lambda\left\|\nabla_{x}[a, \mathbf{b}, c]\right\|^{2}+\lambda \sum_{1 \leq|\vartheta| \leq N}\left\|\nabla_{x} \partial^{\vartheta}[a, \mathbf{b}, c]\right\|^{2}
$$




$$
\leq C \alpha^{2} \sum_{1 \leq|\vartheta| \leq N}\left\|w_{l} \partial^{\vartheta} g_{2}\right\|_{L^{\infty}}^{2}+C \sum_{1 \leq|\vartheta| \leq N}\left\{\left\|\partial^{\vartheta} \mathbf{P}_{1} g_{2}\right\|^{2}+\left\|w_{l} \partial^{\vartheta} g_{1}\right\|_{L^{\infty}}^{2}\right\}
$$

where the Poincaré's inequality $\left\|\nabla_{x}[a, \mathbf{b}, c]\right\| \leq C\left\|\nabla_{x}^{2}[a, \mathbf{b}, c]\right\|$ has been also used.

Step 2.3. Higher order estimates for $\mathbf{P}_{1} g_{2}$. With the above estimates in our hands, we then turn to obtain the higher order $L^{2}$ estimates on $\mathbf{P}_{1} g_{2}$. For this, letting $1 \leq|\vartheta| \leq N$, we take the inner product of $\partial^{\vartheta}(3.35)$ with $\partial^{\vartheta} g_{2}$ and apply Lemma 4.3 so as to obtain

$$
\begin{aligned}
& \sum_{1 \leq|\vartheta| \leq N} \frac{d}{d t}\left\|\partial^{\vartheta} g_{2}\right\|^{2}+\delta_{0} \sum_{1 \leq|\vartheta| \leq N}\left\|\partial^{\vartheta} \mathbf{P}_{1} g_{2}\right\|_{v}^{2} \\
& \leq\left(C \alpha^{2}+\eta+\lambda_{0}\right) \sum_{1 \leq|\vartheta| \leq N}\left\|\mathbf{P}_{0} \partial^{\vartheta} g_{2}\right\|^{2}+C \alpha^{2} \sum_{1 \leq|\vartheta| \leq N}\left\|w_{l} \partial^{\vartheta} g_{2}\right\|_{L^{\infty}}^{2} \\
& \quad+C_{\eta} \sum_{1 \leq|\vartheta| \leq N}\left\|w_{l} \partial^{\vartheta} g_{1}\right\|_{\infty^{\prime}}^{2}
\end{aligned}
$$

where according to Lemma 4.2 and the a priori assumption 3.8, the following estimate has been used:

$$
\begin{aligned}
& \left|\left(\partial^{\vartheta} \Gamma\left(g_{2}, g_{2}\right), \partial^{\vartheta} g_{2}\right)\right|=\left|\left(\partial^{\vartheta} \Gamma\left(g_{2}, g_{2}\right), \partial^{\vartheta} \mathbf{P}_{1} g_{2}\right)\right| \\
\leq & \eta\left\|\partial^{\vartheta} \mathbf{P}_{1} g_{2}\right\|_{v}^{2}+C_{\eta} \int_{\mathbb{T}^{3}}\left\|v^{\frac{1}{2}} \partial^{\vartheta^{\prime}} g_{2}\right\|_{L_{v}^{2}}^{2}\left\|v^{\frac{1}{2}} \partial^{\vartheta-\vartheta^{\prime}} g_{2}\right\|_{L_{v}^{2}}^{2} d x \\
\leq & \eta\left\|\partial^{\vartheta} \mathbf{P}_{1} g_{2}\right\|_{v}^{2}+C_{\eta}\left\|w_{l} \partial^{\vartheta^{\prime}} g_{2}\right\|_{L^{\infty}}^{2}\left\|w_{l} \partial^{\vartheta-\vartheta^{\prime}} g_{2}\right\|_{L^{\infty}}^{2} \\
\leq & \eta\left\|\partial^{\vartheta} \mathbf{P}_{1} g_{2}\right\|_{\nu}^{2}+C_{\eta} \alpha^{2} \sum_{1 \leq|\vartheta| \leq N}\left\|w_{l} \partial^{\vartheta} g_{2}\right\|_{L^{\infty}}^{2}
\end{aligned}
$$

with $l \geq 2$ required.

On the other hand, from (3.28) and (3.29), it follows

$$
\begin{aligned}
& \left\|\left[a_{2}, \mathbf{b}_{2}, c_{2}\right]\right\| \leq\|[a, \mathbf{b}, c]\|+C\left\|w_{l} g_{1}\right\|_{\infty^{\prime}} \\
& \|[a, \mathbf{b}]\| \leq C\left\|\nabla_{x}[a, \mathbf{b}]\right\| \leq C\left\|\nabla_{x} g_{2}\right\|+C\left\|w_{l} \nabla_{x} g_{1}\right\|_{\infty},
\end{aligned}
$$

for $l>\frac{5}{2}$. In addition, by (3.45), we have for $|\vartheta| \leq N-1$

$$
\begin{aligned}
& \left|\kappa_{1} \mathcal{E}_{a}^{i n t}+\mathcal{E}_{\mathbf{b}}^{\text {int }}+\mathcal{E}_{c}^{i n t}\right| \\
\leq & \left\|\nabla_{x} \partial^{\vartheta}[a, \mathbf{b}, c]\right\|^{2}+C\left\|\nabla_{x} \partial^{\vartheta} g_{2}\right\|^{2}+C\left\|w_{l} \nabla_{x} \partial^{\vartheta} g_{1}\right\|_{\infty}^{2} .
\end{aligned}
$$


Let $\kappa_{2}>0$ be suitably small, then we define

$$
\mathcal{E}_{N}^{h}(t)=\kappa_{2}\left(\kappa_{1} \mathcal{E}_{a}^{i n t}+\mathcal{E}_{\mathbf{b}}^{i n t}+\mathcal{E}_{c}^{i n t}\right)+\sum_{1 \leq|\vartheta| \leq N}\left\|\partial^{\vartheta} g_{2}\right\|^{2},
$$

and hence there exist positive constants $\tilde{C}_{1}$ and $\tilde{C}_{2}$ such that

$$
\begin{gathered}
\sum_{1 \leq|\vartheta| \leq N}\left\|\partial^{\vartheta} g_{2}\right\|^{2}-\tilde{C}_{1} \sum_{1 \leq|\vartheta| \leq N}\left\|w_{l} \partial^{\vartheta} g_{1}\right\|_{\infty}^{2} \\
\leq \mathcal{E}_{N}^{h} \leq \sum_{1 \leq|\vartheta| \leq N}\left\|\partial^{\vartheta} g_{2}\right\|^{2}+\tilde{C}_{2} \sum_{1 \leq|\vartheta| \leq N}\left\|w_{l} \partial^{\vartheta} g_{1}\right\|_{\infty}^{2} .
\end{gathered}
$$

By this, (3.46) and (3.47) lead us to

$$
\begin{aligned}
& \frac{d}{d t} \mathcal{E}_{N}^{h}(t)+\lambda \mathcal{E}_{N}^{h}(t) \\
\leq & C \alpha^{2} \sum_{1 \leq|\vartheta| \leq N}\left\|w_{l} \partial^{\vartheta} g_{2}\right\|_{L^{\infty}}^{2}+C \sum_{1 \leq|\vartheta| \leq N}\left\|w_{l} \partial^{\vartheta^{\prime}} g_{1}\right\|_{L^{\infty}}^{2},
\end{aligned}
$$

which further gives

$$
\begin{aligned}
& \sup _{0 \leq s \leq t}\left\|\left[a_{2}, b_{2}\right](s)\right\|^{2}+\sup _{0 \leq s \leq t} \sum_{1 \leq|\vartheta| \leq N}\left\|\partial^{\vartheta} g_{2}(s)\right\|^{2} \\
& \leq C \alpha^{2} \sup _{0 \leq s \leq t} \sum_{1 \leq|\vartheta| \leq N}\left\|w_{l} \partial^{\vartheta} g_{2}\right\|_{L^{\infty}}^{2}+C \sup _{0 \leq s \leq t} \sum_{1 \leq|\vartheta| \leq N}\left\|w_{l} \partial^{\vartheta} g_{1}(s)\right\|_{L^{\infty}}^{2}
\end{aligned}
$$

where (3.48) has been used. As a consequence, (3.25a) and (3.49) imply

$$
\begin{aligned}
& \sup _{0 \leq s \leq t} \sum_{1 \leq|\vartheta| \leq N}\left\|w_{l} \partial^{\vartheta} g_{1}(s)\right\|_{L^{\infty}}+\sup _{0 \leq s \leq t} \sum_{1 \leq|\vartheta| \leq N}\left\|w_{l} \partial^{\vartheta} g_{2}(s)\right\|_{L^{\infty}} \\
& \leq C \sum_{1 \leq|\vartheta| \leq N}\left\|w_{l} \partial^{\vartheta} f_{0}\right\|_{L^{\infty}} .
\end{aligned}
$$

Thus, we get from (3.41) and (3.50) that

$$
\begin{aligned}
& \quad \sup _{0 \leq s \leq t}\left\|w_{l} g_{1}(s)\right\|_{L^{\infty}}+\alpha \sup _{0 \leq s \leq t}\left\|w_{l} g_{2}(s)\right\|_{L^{\infty}} \\
& \quad+\sup _{0 \leq s \leq t} \sum_{1 \leq|\vartheta| \leq N}\left\|w_{l} \partial^{\vartheta} g_{1}(s)\right\|_{L^{\infty}}+\sup _{0 \leq s \leq t} \sum_{1 \leq|\vartheta| \leq N}\left\|w_{l} \partial^{\vartheta} g_{2}(s)\right\|_{L^{\infty}} \\
& \leq C \sum_{|\vartheta| \leq N}\left\|w_{l} \partial^{\vartheta} f_{0}\right\|_{L^{\infty}} .
\end{aligned}
$$


Step 2.4. The estimates for mixture derivatives. In this final sub-step, we shall deduce the $L^{2}$ estimates on $\partial_{\zeta}^{\vartheta} g_{2}$ with $\zeta>0$ and $|\zeta|+|\vartheta| \leq N$. To see this, we first get from the inner product of $\partial_{\zeta}^{\vartheta}(3.35)$ and $\partial_{\zeta}^{\vartheta} g_{2}$ over $(x, v) \in \mathbb{T}^{3} \times \mathbb{R}^{3}$ that

$$
\begin{gathered}
\left(\partial_{t} \partial_{\zeta}^{\vartheta} g_{2}, \partial_{\zeta}^{\vartheta} g_{2}\right)+\left(\partial_{\zeta}^{\vartheta}\left(v \cdot \nabla_{x} g_{2}\right), \partial_{\zeta}^{\vartheta} g_{2}\right)-\beta\left(\partial_{\zeta}^{\vartheta}\left(\nabla_{v} \cdot\left(v g_{2}\right)\right), \partial_{\zeta}^{\vartheta} g_{2}\right) \\
-\alpha\left(\partial_{\zeta}^{\vartheta} \nabla_{v} \cdot\left(A v g_{2}\right), \partial_{\zeta}^{\vartheta} g_{2}\right)-\lambda_{0}\left(\partial_{\zeta}^{\vartheta} g_{2}, \partial_{\zeta}^{\vartheta} g_{2}\right)+\left(\partial_{\zeta}^{\vartheta} L g_{2}, \partial_{\zeta}^{\vartheta} g_{2}\right) \\
=\left(\partial_{\zeta}^{\vartheta}\left(\left(1-\chi_{M}\right) \mu^{-\frac{1}{2}} \mathcal{K} g_{1}\right), \partial_{\zeta}^{\vartheta} g_{2}\right)+e^{-\lambda_{0} t}\left(\partial_{\zeta}^{\vartheta} \Gamma\left(g_{2}, g_{2}\right), \partial_{\zeta}^{\vartheta} g_{2}\right),
\end{gathered}
$$

which gives

$$
\begin{aligned}
& \quad \frac{d}{d t}\left\|\partial_{\zeta}^{\vartheta} g_{2}\right\|^{2}+2\left(\delta_{1}-\lambda_{0}\right)\left\|\partial_{\zeta}^{\vartheta} g_{2}\right\|_{v}^{2} \\
& \leq C\left\|\partial^{\vartheta} g_{2}\right\|^{2}+C \sum_{\substack{\left|\zeta^{\prime}\right|+\left|\vartheta^{\prime}\right| \leq N \\
\zeta^{\prime}<\zeta}}\left\|\partial_{\zeta^{\prime}}^{\vartheta^{\prime}} g_{2}\right\|^{2}+C\left(\alpha+\alpha^{2}\right) \sum_{|\zeta|+|\vartheta| \leq N}\left\|w_{l} \partial_{\zeta}^{\vartheta} g_{2}\right\|_{L^{\infty}}^{2} \\
& \quad+\eta\left\|\partial_{\zeta}^{\vartheta} g_{2}\right\|^{2}+C_{\eta} \sum_{\zeta^{\prime} \leq \zeta}\left\|w_{l} \partial_{\zeta^{\prime}}^{\vartheta} g_{1}\right\|_{L^{\infty}}^{2},
\end{aligned}
$$

according to Lemmas 4.3 and 4.2. Thus, it follows by Gronwall's inequality

$$
\begin{aligned}
& \sup _{0 \leq s \leq t}\left\|\partial_{\zeta}^{\vartheta} g_{2}(s)\right\|^{2} \\
& \leq C \sup _{0 \leq s \leq t}\left\|\partial^{\vartheta} g_{2}(s)\right\|^{2}+C \sup _{\substack{0 \leq s \leq t|| \zeta^{\prime}|+| \vartheta^{\prime} \mid \leq N}}\left\|\partial_{\zeta^{\prime}<\zeta}^{\vartheta^{\prime}} g_{2}\right\|^{2} \\
& \quad+C\left(\alpha+\alpha^{2}\right) \sup _{0 \leq s \leq t|\zeta|+|\vartheta| \leq N} \sum_{\substack{\zeta_{l}\\
}}\left\|w_{l} \partial_{\zeta}^{\vartheta} g_{2}\right\|_{L^{\infty}}^{2}+C \sup _{0 \leq s \leq t \zeta^{\prime} \leq \zeta}\left\|w_{l} \partial_{\zeta^{\prime}}^{\vartheta} g_{1}(s)\right\|_{L^{\infty}}^{2},
\end{aligned}
$$

which further implies

$$
\begin{aligned}
& \sup _{0 \leq s \leq t|\zeta|+|\vartheta| \leq N} \sum_{\substack{\zeta>0 \\
\leq C}}\left\|\partial_{\zeta}^{\vartheta} g_{2}(s)\right\|^{2} \\
& \sup _{|\vartheta| \leq N}\left\|\partial^{\vartheta} g_{2}(s)\right\|^{2}+C \sup _{0 \leq s \leq t} \sum_{|\zeta|+|\vartheta| \leq N}\left\|w_{l} \partial_{\zeta}^{\vartheta} g_{1}(s)\right\|_{L^{\infty}}^{2} \\
& \quad+C\left(\alpha+\alpha^{2}\right) \sum_{|\zeta|+|\vartheta| \leq N}\left\|w_{l} \partial_{\zeta}^{\vartheta} g_{2}\right\|_{L^{\infty}}^{2} .
\end{aligned}
$$

On the other hand, from (3.22) and (3.23), it follows

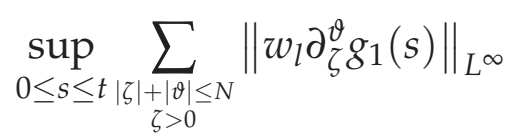




$$
\begin{aligned}
& \leq \sum_{|\zeta|+|\vartheta| \leq N}\left\|w_{l} \partial_{\zeta}^{\vartheta} f_{0}\right\|_{L^{\infty}}+C \alpha \sup _{0 \leq s \leq t|\zeta|+|\vartheta| \leq N} \sum_{\zeta>0}\left\|w_{l} \partial_{\zeta}^{\vartheta} g_{2}(s)\right\|_{L^{\infty}} \\
& \quad+C \alpha \sup _{0 \leq s \leq t|\vartheta| \leq N}\left\|w_{l} \partial^{\vartheta} g_{2}(s)\right\|_{L^{\infty}} \\
& \quad \sup _{0 \leq s \leq t|\zeta|+|\vartheta| \leq N}\left\|w_{l} \partial_{\zeta>0}^{\vartheta} g_{2}(s)\right\|_{L^{\infty}} \\
& \leq C \sup _{0 \leq s \leq t|\zeta|+|\vartheta| \leq N} \sum_{\substack{\zeta>0\\
}}\left\|w_{l} \partial_{\zeta}^{\vartheta} g_{1}(s)\right\|_{L^{\infty}}+C \sup _{0 \leq s \leq t|\zeta|+|\vartheta| \leq N}\left\|\sum_{\zeta>0}^{\vartheta} g_{2}(s)\right\| \\
& \quad+C \sup _{0 \leq s \leq t|\vartheta| \leq N}\left\|w_{l} \partial^{\vartheta} g_{1}(s)\right\|_{L^{\infty}}+C \sup _{0 \leq s \leq t|\vartheta| \leq N} \sum_{0 \leq w_{l} \partial^{\vartheta} g_{2}(s) \|_{L^{\infty}} .}
\end{aligned}
$$

Now (3.51)-(3.54) lead us to

$$
\begin{aligned}
& \sup _{0 \leq s \leq t|\zeta|+|\vartheta| \leq N} \sum_{\leq}\left\|w_{l} \partial_{\zeta}^{\vartheta} g_{1}(s)\right\|_{L^{\infty}}+\alpha \sup _{0 \leq s \leq t|\zeta|+|\vartheta| \leq N} \sum_{|\zeta|+|\vartheta| \leq N}\left\|w_{l} \partial_{\zeta}^{\vartheta} g_{2}(s)\right\|_{L^{\infty}} \\
& \leq C \sum_{l} \partial_{\zeta}^{\vartheta} f_{0} \|_{L^{\infty}} .
\end{aligned}
$$

Thus, (3.7) is valid and this also confirms (1.17).

Finally, by the similar procedure as that of [14, Step 4, pp.47], one can show that the solution of (1.13) and (1.14) is non-negative, and the details of the proof is omitted for brevity. This ends the proof of Theorem 1.2.

\section{Appendix}

In this section, we provide those estimates that have been used in the previous sections. We will first give the basic estimates on the linearized operator $L$ as well as the nonlinear operators $\Gamma$ and $Q$, then present a key estimate for the operator $\mathcal{K}$ in the case of hard potentials, and in the end derive a lower bound for a matrix exponential.

The following lemma is concerned with the integral operator $K$ given by (2.3), and its proof in case of the hard sphere model $(\gamma=1)$ has been given by [24, Lemma 3].

Lemma 4.1. Let $K$ be defined as (2.3), then it holds that

$$
K f(v)=\int_{\mathbb{R}^{3}} \mathbf{k}\left(v, v_{*}\right) f\left(v_{*}\right) d v_{*}
$$


with

$$
\left|\mathbf{k}\left(v, v_{*}\right)\right| \leq C\left\{\left|v-v_{*}\right|^{\gamma}+\left|v-v_{*}\right|^{-2+\gamma}\right\} e^{-\frac{1}{8}\left|v-v_{*}\right|^{2}-\frac{1}{8} \frac{|v|^{2}-\left.\left|v_{*}\right|^{2}\right|^{2}}{\left|v-v_{*}\right|^{2}}} .
$$

Moreover, let

$$
\mathbf{k}_{w}\left(v, v_{*}\right)=w_{l}(v) \mathbf{k}\left(v, v_{*}\right) w_{l}^{-1}\left(v_{*}\right)
$$

with $l \geq 0$, then it also holds that

$$
\int_{\mathbb{R}^{3}} \mathbf{k}_{w}\left(v, v_{*}\right) e^{\frac{\varepsilon\left|v-v_{*}\right|^{2}}{8}} d v_{*} \leq \frac{C}{1+|v|}
$$

for $\varepsilon=0$ or any $\varepsilon>0$ small enough.

For the velocity weighted derivative estimates on the nonlinear operator $\Gamma$, one has

Lemma 4.2. Let $0 \leq \gamma \leq 1$ and $\theta \in[0,1]$. For any $p \in[1,+\infty]$ and any $l \geq 0$, it holds that

$$
\begin{aligned}
& \left\|w_{l} v^{-\theta} \partial_{\zeta} \Gamma(f, g)\right\|_{L_{v}^{p}} \\
\leq & C \sum_{\zeta^{\prime}+\zeta^{\prime \prime} \leq \zeta}\left\{\left\|w_{l} v^{1-\theta} \partial_{\zeta^{\prime}} f\right\|_{L_{v}^{p}}\left\|\partial_{\zeta^{\prime \prime}} g\right\|_{L_{v}^{p}}+\left\|\partial_{\zeta^{\prime}} f\right\|_{L_{v}^{p}}\left\|w_{l} v^{1-\theta} \partial_{\zeta^{\prime \prime}} g\right\|_{L_{v}^{p}}\right\} .
\end{aligned}
$$

Proof. Note that if $l=0$ and $\zeta=0,(4.1)$ was given in [29, Theorem 1.2.3]. Let us now show that (4.1) can be generalized to $l \geq 0$ and $\zeta \geq 0$. For this, we first have from definition (2.2) that

$$
\begin{aligned}
\partial_{\zeta} \Gamma(f, g)= & \partial_{\zeta} \int_{\mathbb{R}^{3}} \int_{\mathbb{S}^{2}} B_{0}(\cos \theta)\left|v-v_{*}\right|^{\gamma} \mu^{\frac{1}{2}}\left(v_{*}\right) f\left(v_{*}^{\prime}\right) g\left(v^{\prime}\right) d \omega d v_{*} \\
& -\partial_{\zeta} \int_{\mathbb{R}^{3}} \int_{\mathbb{S}^{2}} B_{0}(\cos \theta)\left|v-v_{*}\right|^{\gamma} \mu^{\frac{1}{2}}\left(v_{*}\right) f\left(v_{*}\right) g(v) d \omega d v_{*} \\
= & \partial_{\zeta} \int_{\mathbb{R}^{3}} \int_{\mathbb{S}^{2}} B_{0}(\cos \theta)\left|v-v_{*}\right|^{\gamma} \mu^{\frac{1}{2}}\left(v_{*}\right) f\left(v_{*}^{\prime}\right) g\left(v^{\prime}\right) d \omega d v_{*} \\
& -c_{0} \partial_{\zeta}\left[g(v) \int_{\mathbb{R}^{3}}\left|v-v_{*}\right|^{\gamma} \mu^{\frac{1}{2}}\left(v_{*}\right) f\left(v_{*}\right) d v_{*}\right],
\end{aligned}
$$

where we have used $\int_{\mathrm{S}^{2}} B_{0}(\cos \theta) d \omega=c_{0}$ for a constant $c_{0}>0$. Then, by a change of variable $v_{*}-v \rightarrow u$, one has

$$
\begin{aligned}
& \partial_{\zeta} \Gamma(f, g) \\
& =\underbrace{\sum_{\zeta^{\prime \prime} \leq \zeta^{\prime} \leq \zeta} C_{\zeta}^{\zeta^{\prime}} C_{\zeta^{\prime}}^{\zeta^{\prime \prime}} \int_{\mathbb{R}^{3}} \int_{S^{2}} B_{0}(\cos \theta)|u|^{\gamma}\left(\partial_{\zeta-\zeta^{\prime}} \mu^{\frac{1}{2}}\right)(u+v)\left(\partial_{\zeta^{\prime}-\zeta^{\prime \prime}} f\right)\left(v+u_{\perp}\right)\left(\partial_{\zeta^{\prime \prime}} g\right)\left(v+u_{\|}\right) d \omega d u}_{\Gamma_{1}}
\end{aligned}
$$




$$
\underbrace{-c_{0} \sum_{\zeta^{\prime} \leq \zeta} C_{\zeta}^{\zeta^{\prime}}\left[\left(\partial_{\zeta^{\prime}} g\right)(v) \int_{\mathbb{R}^{3}}|u|^{\gamma}\left(\partial_{\zeta-\zeta^{\prime}} \mu^{\frac{1}{2}}\right)(v+u)\left(\partial_{\zeta^{\prime}} f\right)(v+u) d u\right]}_{\Gamma_{2}},
$$

where $u_{\|}=(u \cdot \omega) \omega$ and $u_{\perp}=u-u_{\|}$. As

$$
\left|\left(\partial_{\zeta-\zeta^{\prime}} \mu^{\frac{1}{2}}\right)(u+v)\right| \leq C \mu^{\frac{1}{4}}(u+v),
$$

one has by changing variable $u$ back to $v_{*}-v$ that

$$
\begin{aligned}
\left|\Gamma_{1}\right| & \leq C \int_{\mathbb{R}^{3}} \int_{\mathbb{S}^{2}} B_{0}(\cos \theta)|u|^{\gamma} \mu^{\frac{1}{4}}(u+v)\left|\left(\partial_{\zeta^{\prime}-\zeta^{\prime \prime}} f\right)\left(v+u_{\perp}\right)\left(\partial_{\zeta^{\prime \prime}} g\right)\left(v+u_{\|}\right)\right| d \omega d u \\
& =C \int_{\mathbb{R}^{3}} \int_{\mathbb{S}^{2}} B_{0}(\cos \theta)\left|v_{*}-v\right|^{\gamma} \mu^{\frac{1}{4}}\left(v_{*}\right)\left|\left(\partial_{\zeta^{\prime}-\zeta^{\prime \prime}} f\right)\left(v_{*}^{\prime}\right)\left(\partial_{\zeta^{\prime \prime}} g\right)\left(v^{\prime}\right)\right| d \omega d v_{*},
\end{aligned}
$$

which together with the inequality

$$
\left(w_{l} v^{-\theta+1}\right)(v) \leq C\left(\left(w_{l} v^{-\theta+1}\right)\left(v^{\prime}\right)+\left(w_{l} v^{-\theta+1}\right)\left(v_{*}^{\prime}\right)\right)
$$

implies

$$
\begin{aligned}
w_{l} v^{-\theta}\left|\Gamma_{1}\right| \leq & C\left(\left(w_{l} v^{-\theta+1}\right)\left(v^{\prime}\right)+\left(w_{l} v^{-\theta+1}\right)\left(v_{*}^{\prime}\right)\right) v^{-1}(v) \\
& \times \int_{\mathbb{R}^{3}} \int_{S^{2}} B_{0}(\cos \theta)\left|v_{*}-v\right|^{\gamma} \mu^{\frac{1}{4}}\left(v_{*}\right)\left|\left(\partial_{\zeta^{\prime}-\zeta^{\prime \prime}} f\right)\left(v_{*}^{\prime}\right)\left(\partial_{\zeta^{\prime \prime}} g\right)\left(v^{\prime}\right)\right| d \omega d v v_{*} \\
\leq & C\left\{\left\|w_{l} v^{-\theta+1} \partial_{\zeta^{\prime}-\zeta^{\prime \prime}} f\right\|_{L^{\infty}}\left\|\partial_{\zeta^{\prime \prime}} g\right\|_{L^{\infty}}+\left\|\partial_{\zeta^{\prime}-\zeta^{\prime \prime}} f\right\|_{L^{\infty}}\left\|w_{l} v^{-\theta+1} \partial_{\zeta^{\prime \prime}} g\right\|_{L^{\infty}}\right\} \\
& \times v^{-1}(v) \int_{\mathbb{R}^{3}} \int_{S^{2}} B_{0}(\cos \theta)\left|v_{*}-v\right|^{\gamma} \mu^{\frac{1}{4}}\left(v_{*}\right) d \omega d v_{*} \\
\leq & C\left\{\left\|w_{l} v^{-\theta+1} \partial_{\zeta^{\prime}-\zeta^{\prime \prime}} f\right\|_{L^{\infty}}\left\|\partial_{\zeta^{\prime \prime}} g\right\|_{L^{\infty}}+\left\|\partial_{\zeta^{\prime}-\zeta^{\prime \prime}} f\right\|_{L^{\infty}}\left\|w_{l} v^{-\theta+1} \partial_{\zeta^{\prime \prime}} g\right\|_{L^{\infty}}\right\} .
\end{aligned}
$$

This confirms the $L^{\infty}$ estimate for $\Gamma_{1}$. If $p \in[1, \infty)$, by Hölder's inequality, we get

$$
\begin{aligned}
w_{l} v^{-\theta}\left|\Gamma_{1}\right| \leq & C_{\zeta} w_{l} v^{-\theta}\left(\int_{\mathbb{R}^{3}} \int_{\mathbb{S}^{2}} B_{0}(\cos \theta)\left|v_{*}-v\right|^{p^{\prime}} \mu^{\frac{p^{\prime}}{4}}\left(v_{*}\right) d \omega d v_{*}\right)^{\frac{1}{p^{\prime}}} \\
& \times\left(\int_{\mathbb{R}^{3}} \int_{\mathrm{S}^{2}} B_{0}(\cos \theta) \mid\left(\left(\partial_{\zeta^{\prime}-\zeta^{\prime \prime}} f\right)\left(v_{*}^{\prime}\right)\left(\partial_{\zeta^{\prime \prime}} g\right)\left(v^{\prime}\right) \mid\right)^{p} d \omega d v_{*}\right)^{\frac{1}{p}} \\
\leq & C_{\zeta} w_{l} v^{1-\theta}\left(\int_{\mathbb{R}^{3}}\left|\left(\partial_{\zeta^{\prime}-\zeta^{\prime \prime}} f\right)\left(v_{*}^{\prime}\right)\left(\partial_{\zeta^{\prime \prime}} g\right)\left(v^{\prime}\right)\right|^{p} d v_{*}\right)^{\frac{1}{p}}
\end{aligned}
$$


where $\frac{1}{p}+\frac{1}{p^{\prime}}=1$. Therefore, using (4.3) again and by a change of variable $\left(v^{\prime}, v_{*}^{\prime}\right) \rightarrow$ $\left(v, v_{*}\right)$, one has

$$
\begin{aligned}
\left\|w_{l} v^{-\theta} \Gamma_{1}\right\|_{L^{p}}^{p} \leq & \int_{\mathbb{R}^{3}} w_{l}^{p} v^{-p \theta+p} \int_{\mathbb{R}^{3}}\left|\left(\partial_{\zeta^{\prime}-\zeta^{\prime \prime}} f\right)\left(v_{*}^{\prime}\right)\left(\partial_{\zeta^{\prime \prime}} g\right)\left(v^{\prime}\right)\right|^{p} d v_{*} d v \\
= & \int_{\mathbb{R}^{3}} \int_{\mathbb{R}^{3}}\left(w_{l}^{p} v^{-p \theta+p}\right)\left(v_{*}^{\prime}\right)\left|\left(\partial_{\zeta^{\prime}-\zeta^{\prime \prime}} f\right)\left(v_{*}\right)\left(\partial_{\zeta^{\prime \prime}} g\right)(v)\right|^{p} d v_{*} d v \\
\leq & \int_{\mathbb{R}^{3}} \int_{\mathbb{R}^{3}}\left[\left(w_{l}^{p} v^{-p \theta+p}\right)(v)+\left(w_{l}^{p} v^{-p \theta+p}\right)\left(v_{*}\right)\right] \\
& \times\left|\left(\partial_{\zeta^{\prime}-\zeta^{\prime \prime}} f\right)\left(v_{*}\right)\left(\partial_{\zeta^{\prime \prime}} g\right)(v)\right|^{p} d v_{*} d v \\
\leq & C\left\{\left\|w_{l} v^{-\theta} \partial_{\zeta^{\prime}-\zeta^{\prime \prime}} f\right\|_{L_{v}^{p}}^{p}\left\|\partial_{\zeta^{\prime \prime}}\right\|_{L_{v}^{p}}^{p}+\left\|\partial_{\zeta^{\prime}-\zeta^{\prime \prime}} f\right\|_{L_{v}^{p}}^{p}\left\|w_{l} v^{-\theta} \partial_{\zeta^{\prime \prime} g}\right\|_{L_{v}^{p}}^{p}\right\} .
\end{aligned}
$$

The corresponding estimates for $\Gamma_{2}$ are similar and easier, so we omit them for brevity. This completes the proof of Lemma 4.2.

The following lemma is concerned with coercivity estimates for the linear collision operator $L$.

Lemma 4.3. Let $0 \leq \gamma \leq 1$, then there is a constant $\delta_{0}>0$ such that

$$
\langle L f, f\rangle=\left\langle L \mathbf{P}_{1} f, \mathbf{P}_{1} f\right\rangle \geq \delta_{0}\left\|\mathbf{P}_{1} f\right\|_{v}^{2}
$$

where $\|\cdot\|_{v}=\left\|v^{\frac{1}{2}} \cdot\right\|$. Moreover, there are constants $\delta_{1}>0$ and $C>0$ such that for $|\zeta|>0$

$$
\left\langle\partial_{\zeta} L f, \partial_{\zeta} f\right\rangle \geq \delta_{1}\left\|\partial_{\zeta} f\right\|_{v}^{2}-C\|f\|^{2} .
$$

Proof. Note that (4.4) has been already proved in [23, Lemma 3.2]. As for (4.5), from [23, Lemma 3.3], we have

$$
\left\langle\partial_{\zeta} L f, \partial_{\zeta} f\right\rangle \geq \delta_{1}\left\|\partial_{\zeta} f\right\|_{v}^{2}-C\|f\|_{v}^{2} .
$$

We now prove that this can be relaxed to (4.5), which is indeed true for Maxwell molecular case because $v \sim c_{0}$ for some $c_{0}>0$ in this situation. For $0<\gamma \leq 1$, we write

$$
\begin{aligned}
& \left\langle\partial_{\zeta} L f, \partial_{\zeta} f\right\rangle=\left\langle\partial_{\zeta}(v f), \partial_{\zeta} f\right\rangle-\left\langle\partial_{\zeta}(K f), \partial_{\zeta} f\right\rangle \\
= & \left\langle L \partial_{\zeta} f, \partial_{\zeta} f\right\rangle+\sum_{0<\zeta^{\prime} \leq \zeta} C_{\zeta}^{\zeta^{\prime}}\left\langle\partial_{\zeta^{\prime}} \nu \partial_{\zeta-\zeta^{\prime}} f, \partial_{\zeta} f\right\rangle-\sum_{0<\zeta^{\prime} \leq \zeta} C_{\zeta}^{\zeta^{\prime}}\left\langle\left(\partial_{\zeta^{\prime}} K\right) \partial_{\zeta-\zeta^{\prime}} f, \partial_{\zeta} f\right\rangle .
\end{aligned}
$$

From (4.4), one has

$$
\left\langle L \partial_{\zeta} f, \partial_{\zeta} f\right\rangle \geq \delta_{0}\left\|\mathbf{P}_{1} \partial_{\zeta} f\right\|_{v}^{2} \geq \delta_{0}\left\|\partial_{\zeta} f\right\|_{v}^{2}-\delta_{0}\left\|\mathbf{P}_{0} \partial_{\zeta} f\right\|_{v}^{2} \geq \delta_{0}\left\|\partial_{\zeta} f\right\|_{v}^{2}-C\|f\|^{2} .
$$


By definition (2.3), it follows

$$
\mathbf{1}_{\zeta^{\prime}>0}\left|\partial_{\zeta^{\prime}} v\right| \leq C(1+|v|)^{\gamma-\left|\zeta^{\prime}\right|} \leq C
$$

Thus, one has by Cauchy-Schwarz's inequality with $\eta>0$ and Sobolev's interpolation inequality that

$$
\left|\left\langle\partial_{\zeta^{\prime}} v \partial_{\zeta-\zeta^{\prime}} f, \partial_{\zeta} f\right\rangle\right| \leq \eta\left\|\partial_{\zeta} f\right\|^{2}+C_{\eta}\|f\|^{2}
$$

Next, in view of (2.4), we have by a change of variable $v_{*}-v \rightarrow u$

$$
\begin{aligned}
& \left(\partial_{\zeta^{\prime}} K\right) \partial_{\zeta-\zeta^{\prime}} f \\
= & \tilde{c}_{1} \sum_{0 \leq \zeta^{\prime} \leq \zeta} C_{\zeta}^{\zeta^{\prime}} \int_{\mathbb{R}^{3} \times \omega^{2}} B_{0}(\cos \theta)|u|^{\gamma} \partial_{\zeta^{\prime}}\left\{e^{-\frac{|u+v|^{2}+|v|^{2}}{4}}\right\} \partial_{\zeta-\zeta^{\prime}} f(v+u) d u \\
& \quad-\tilde{c}_{2} \sum_{0 \leq \zeta^{\prime} \leq \zeta} C_{\zeta}^{\zeta^{\prime}} \int_{\mathbb{R}^{3} \times \omega^{2}} B_{0}(\cos \theta)|u|^{\gamma} \partial_{\zeta^{\prime}}\left\{e^{-\frac{1}{8}|u|^{2}-\frac{1}{8} \frac{\left.\left.|2 v \cdot u+| u\right|^{2}\right|^{2}}{|u|^{2}}}\right\} \partial_{\zeta-\zeta^{\prime}} f(v+u) d u .
\end{aligned}
$$

Furthermore, direct computations give

$$
\begin{aligned}
& \partial_{\zeta^{\prime}}\left\{e^{-\frac{|u+v|^{2}+|v|^{2}}{4}}\right\} \leq C\left(\zeta^{\prime}\right) e^{-\frac{|u+v|^{2}+|v|^{2}}{8}}, \\
& \partial_{\zeta^{\prime}}\left\{e^{-\frac{1}{8}|u|^{2}-\frac{1}{8} \frac{\left.\left.|2 v \cdot u+| u\right|^{2}\right|^{2}}{|u|^{2}}}\right\} \leq C\left(\zeta^{\prime}\right) e^{-\frac{1}{16}|u|^{2}-\frac{1}{16} \frac{\left.\left.|2 v \cdot u+| u\right|^{2}\right|^{2}}{|u|^{2}}},
\end{aligned}
$$

which further implies

$$
\left|\left(\partial_{\zeta^{\prime}} K\right) \partial_{\zeta-\zeta^{\prime}} f\right| \leq C(\zeta) \int_{\mathbb{R}^{3}} \overline{\mathbf{k}}\left(v, v_{*}\right)\left|\partial_{\zeta-\zeta^{\prime}} f\left(v_{*}\right)\right| d v_{*}
$$

with

$$
\overline{\mathbf{k}}\left(v, v_{*}\right) \leq C\left\{\left|v-v_{*}\right|^{\gamma}+\left|v-v_{*}\right|^{-2+\gamma}\right\} e^{-\frac{1}{16}\left|v-v_{*}\right|^{2}-\frac{1}{16} \frac{|v|^{2}-\left.|v *|^{2}\right|^{2}}{|v-v *|^{2}}} .
$$

In particular,

$$
\int_{\mathbb{R}^{3}} \overline{\mathbf{k}}\left(v, v_{*}\right) d v \leq \frac{C}{1+\left|v_{*}\right|}, \quad \int_{\mathbb{R}^{3}} \overline{\mathbf{k}}\left(v, v_{*}\right) d v_{*} \leq \frac{C}{1+|v|} .
$$

Therefore, by Cauchy-Schwarz's inequality, Fubini's theorem and Sobolev's interpolation inequality, we obtain

$$
\left|\left\langle\left(\partial_{\zeta^{\prime}} K\right) \partial_{\zeta-\zeta^{\prime}} f, \partial_{\zeta} f\right\rangle\right|
$$




$$
\begin{aligned}
& \leq \eta\left\|\partial_{\zeta} f\right\|^{2}+C_{\eta} \int_{\mathbb{R}^{3}}\left(\int_{\mathbb{R}^{3}} \overline{\mathbf{k}}\left(v, v_{*}\right)\left|\partial_{\zeta-\zeta^{\prime}} f\left(v_{*}\right)\right| d v_{*}\right)^{2} d v \\
& \leq \eta\left\|\partial_{\zeta} f\right\|^{2}+C_{\eta} \int_{\mathbb{R}^{3}} \int_{\mathbb{R}^{3}} \overline{\mathbf{k}}\left(v, v_{*}\right) d v_{*} \int_{\mathbb{R}^{3}} \overline{\mathbf{k}}\left(v, v_{*}\right)\left|\partial_{\zeta-\zeta^{\prime}} f\left(v_{*}\right)\right|^{2} d v_{*} d v \\
& \leq \eta\left\|\partial_{\zeta} f\right\|^{2}+C_{\eta} \int_{\mathbb{R}^{3}} \int_{\mathbb{R}^{3}} \overline{\mathbf{k}}\left(v, v_{*}\right) d v\left|\partial_{\zeta-\zeta^{\prime}} f\left(v_{*}\right)\right|^{2} d v_{*} \\
& \leq 2 \eta\left\|\partial_{\zeta} f\right\|^{2}+C_{\eta}\|f\|^{2} .
\end{aligned}
$$

Finally, plugging (4.7), (4.8) and (4.10) into (4.6) gives (4.5). This ends the proof of Lemma 4.3.

Remark 4.1. From (4.9), one can justify that $\partial_{\zeta^{\prime}} K$ is a compact operator from $H^{|\zeta|}$ to $H^{|\zeta|}$, which directly implies (4.10), cf. [22, Lemma 2.2].

Next, the following lemma which was proved in [14, Proposition 3.1] gives the $L^{\infty}$ estimates of the solutions in the case of Maxwell molecule model.

Lemma 4.4. Let $\gamma=0$ and $\mathcal{K}$ be given by (2.5), then for any nonnegative integer $|\zeta| \geq 0$, there is $C>0$ such that for any arbitrarily large $l>0$, there is $M=M(l)>0$ such that it holds that

$$
\sup _{|v| \geq M} w_{l}\left|\partial_{\zeta}(\mathcal{K} f)\right| \leq \frac{C}{l} \sum_{0 \leq \zeta^{\prime} \leq \zeta}\left\|w_{l} \partial_{\zeta^{\prime}} f\right\|_{L^{\infty}} .
$$

In particular, one can choose $M=l^{2}$.

In the case of $0<\gamma \leq 1$, the following lemma which can be found in [1, Proposition 3.1] enables us to gain the smallness property of $\mathcal{K}$ at large velocity.

Lemma 4.5. Let $0 \leq \gamma \leq 1$ and $l>4$, then there exists a function $\varsigma(l)$ which satisfies $\varsigma(l) \rightarrow 0$ as $l \rightarrow+\infty$ such that

$$
\begin{aligned}
& w_{l}\left\{\left|Q_{\text {loss }}(f, g)\right|+\left|Q_{\text {gain }}(f, g)\right|+\left|Q_{\text {gain }}(g, f)\right|\right\} \\
\leq & \left\|w_{l} f\right\|_{L^{\infty}}\left\{C(l)\left\|w_{l+\gamma / 2} g\right\|_{L^{\infty}}+\zeta(l)\left\|w_{3} g\right\|_{L^{\infty}}(1+|v|)^{\gamma}\right\},
\end{aligned}
$$

where $Q_{\text {loss }}$ denotes the negative part of $Q$ in (1.2).

The following result is a direct consequence of Lemma 4.5.

Lemma 4.6. Let $0<\gamma \leq 1$, then there is a constant $C>0$ such that for any arbitrarily large $l>0$, there are sufficiently large $M=M(l)>0$ and suitably small $\varsigma=\varsigma(l)>0$ such that it holds that

$$
\sup _{|v| \geq M} v^{-1} w_{l}|\mathcal{K} f| \leq C\left\{(1+M)^{-\frac{\gamma}{2}}+\varsigma\right\}\left\|w_{l} f\right\|_{L^{\infty}}
$$


Proof. Recall the definition (2.5) for $\mathcal{K}$. Let $g=\mu$ in (4.12), then we obtain

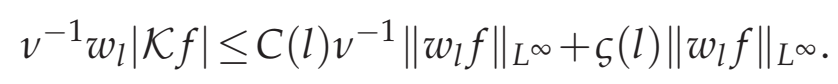

Noticing that $\varsigma(l)=\frac{1}{l}$ according to the proof in [1, Proposition 3.1], we first choose $l$ to be suitably large so that $\zeta$ is small enough, then we set $M>0$ to be sufficiently large such that $C(l)(1+M)^{-\frac{\gamma}{2}} \leq C$ thanks to $\gamma>0$. Then (4.13) follows from (4.14). This concludes the proof of Lemma 4.6.

The following lemma concerning the polynomial weighted estimates on the collision operator $Q$ can be verified by using a parallel argument as for obtaining [1, Proposition 3.1].

Lemma 4.7. For $l>4$ and $\gamma \geq 0$, then it holds that

$$
\left|w_{l} v^{-1} \partial_{\zeta} Q\left(F_{1}, F_{2}\right)\right| \leq C \sum_{\zeta^{\prime}+\zeta^{\prime \prime} \leq \zeta}\left\|w_{l} \partial_{\zeta^{\prime}} F_{1}\right\|_{L^{\infty}}\left\|w_{l} \partial_{\zeta^{\prime \prime}} F_{2}\right\|_{L^{\infty}}
$$

Finally, we give a technical lemma on the determinant of a matrix exponential and we omit the proof for brevity.

Lemma 4.8. Let $\mathcal{M}=\alpha \overline{\mathcal{M}}$, where $\overline{\mathcal{M}}=\left(\bar{a}_{i j}\right) \in M_{3 \times 3}(\mathbb{R})$ is an invertible constant matrix with $\max \left\{\left|\bar{a}_{i j}\right|\right\}=C_{\mathcal{M}}$, and $\alpha>0$ is suitably small.

(i) If $\frac{1}{3} \geq \eta>0$, then it holds that

$$
|| \mathcal{M}^{-1}|| e^{\eta \mathcal{M}}-I|| \geq \frac{\eta^{3}}{8}
$$

(ii) Let $v \in \mathbb{R}^{3}$ be a vector satisfying $|v| \leq M$ with $M>0$, then for any $\eta>0$, it holds that

$$
\left|\mathcal{M}^{-1}\left\{e^{\eta \mathcal{M}}-I\right\} v\right| \leq \eta M e^{3 C_{\mathcal{M}}^{\alpha \eta}} .
$$

\section{Acknowledgements}

Renjun Duan's research was partially supported by the General Research Fund (Project No. 14301720) from RGC of Hong Kong and the Direct Grant (4053397) from CUHK. Shuangqian Liu's research was supported by grants from the National Natural Science Foundation of China (Contracts 11971201, 11731008), and Hong Kong Institute for Advanced Study (No. 9360157). 


\section{References}

[1] L. Arkeryd, R. Esposito and M. Pulvirenti, The Boltzmann equation for weakly inhomogeneous data, Comm. Math. Phys. 111(3) (1987), 393-407.

[2] J. Bedrossian, N. Masmoudi and V. Vicol, Enhanced dissipation and inviscid damping in the inviscid limit of the Navier-Stokes equations near the two dimensional Couette flow, Arch. Ration. Mech. Anal. 219 (2016), 1087-1159.

[3] A. V. Bobylev, On a class of self-similar solutions of the Boltzmann equation, arXiv:2111. 00872.

[4] A. V. Bobylev, The method of the Fourier transform in the theory of the Boltzmann equation for Maxwell molecules, Dokl. Akad. Nauk. SSSR 225(6) (1975), 1041-1044. (in Russian).

[5] A. V. Bobylev, The theory of the nonlinear spatially uniform Boltzmann equation for Maxwellian molecules, Sov. Scient. Rev. Sect. C Math. Phys. Rev. 7 (1988), 111-233.

[6] A. V. Bobylev and C. Cercignani, Moment equations for a granular material in a thermal bath, J. Stat. Phys. 106(3-4) (2002), 547-567.

[7] A. V. Bobylev and C. Cercignani, Exact eternal solutions of the Boltzmann equation, J. Stat. Phys. 106(5-6) (2002), 1019-1039.

[8] A. V. Bobylev and C. Cercignani, Self-similar solutions of the Boltzmann equation and their applications, J. Stat. Phys. 106(5-6) (2002), 1039-1071.

[9] A. V. Bobylev and C. Cercignani, Self-similar asymptotics for the Boltzmann equation with inelastic and elastic interactions, J. Stat. Phys. 110(1-2) (2003), 335-375.

[10] A. V. Bobylev, A. Nota and J. J. L. Velázquez, Self-similar asymptotics for a modified Maxwell-Boltzmann equation in systems subject to deformations, Comm. Math. Phys. 380(1) (2020), 409-448.

[11] R. Caflisch, The Boltzmann equation with a soft potential, II. Nonlinear, spatially-periodic, Comm. Math. Phys. 74(2) (1980), 97-109.

[12] C. Cercignani, Existence of homoenergetic affine flows for the Boltzmann equation, Arch. Ration. Mech. Anal. 105(4) (1989), 377-387.

[13] C. Cercignani, Shear flow of a granular material, J. Stat. Phys. 102(5-6) (2001), 14071415.

[14] R.-J. Duan and S.-Q. Liu, The Boltzmann equation for uniform shear flow, Arch. Ration. Mech. Anal. 242(3) (2021), 1947-2002.

[15] R.-J. Duan, S.-Q. Liu and T. Yang, The Boltzmann equation for plane Couette flow, arXiv: 2107.02458.

[16] R.-J. Duan, F.-M. Huang, Y. Wang and Z. Zhang, Effects of soft interaction and nonisothermal boundary upon long-time dynamics of rarefied gas, Arch. Ration. Mech. Anal. 234(2) (2019), 925-1006.

[17] R. Esposito, Y. Guo, C. Kim and R. Marra, Non-isothermal boundary in the Boltzmann theory and Fourier law, Comm. Math. Phys. 323(1) (2013), 177-239. 
[18] R. Esposito, Y. Guo, C. Kim and R. Marra, Stationary solutions to the Boltzmann equation in the hydrodynamic limit, Ann. PDE 4(1) (2018), pp. 119.

[19] V. S. Galkin, On a class of solutions of Grad's moment equation, J. Appl. Math. Mech. 22 (1958), 532-536.

[20] V. Garzó and A. Santos, Kinetic Theory of Gases in Shear Flows. Nonlinear transport, in: Fundamental Theories of Physics, Vol. 131, Springer, 2003.

[21] R. T. Glassey, The Cauchy Problem in Kinetic Theory, SIAM, 1987.

[22] Y. Guo, The Vlasov-Poisson-Boltzmann system near Maxwellians, Comm. Pure Appl. Math. 55(9) (2002), 1104-1135.

[23] Y. Guo, Boltzmann diffusive limit beyond the Navier-Stokes approximation, Comm. Pure. Appl. Math. 55(9) (2006), 0626-0687.

[24] Y. Guo, Decay and continuity of the Boltzmann equation in bounded domains, Arch. Ration. Mech. Anal. 197(3) (2010), 713-809.

[25] R. D. James, A. Nota and J. J. L. Velázquez, Self-similar profiles for homoenergetic solutions of the Boltzmann equation: particle velocity distribution and entropy, Arch. Ration. Mech. Anal. 231(2) (2019), 787-843.

[26] R. D. James, A. Nota and J. J. L. Velázquez, Long-time asymptotics for homoenergetic solutions of the Boltzmann equation: collision-dominated case, J. Nonlinear Sci. 29(5) (2019), 1943-1973.

[27] R. D. James, A. Nota and J. J. L. Velázquez, Long-time asymptotics for homoenergetic solutions of the Boltzmann equation: hyperbolic-dominated case, Nonlinearity 33(8) (2020), 3781-3815.

[28] C. Truesdell, On the pressures and flux of energy in a gas according to Maxwell's kinetic theory II, J. Rat. Mech. Anal. 5 (1956), 55-128.

[29] S. Ukai and T. Yang, Mathematical Theory of Boltzmann Equation, Lecture Notes Series No. 8 (Liu Bie Ju Centre for Mathematical Sciences), City University of Hong Kong, 2006. 